\title{
Synthesis of Vinyl Isocyanides and Development of a Convertible Isonitrile
}

\author{
Martina Spallarossa, ${ }^{+, *}$ Qian Wang, ${ }^{\dagger}$ Renata Riva, ${ }^{\sharp}$ Jieping Zhu ${ }^{{ }_{+}}$ \\ ${ }^{\dagger}$ Laboratory of Synthesis and Natural Products, Institute of Chemical Sciences and Engineering, \\ Ecole Polytechnique Federale de Lausanne, EPFL-SB-ISIC-LSPN, BCH 5304, 1015 Lausanne, \\ Switzerland \\ \#Department of Chemistry and Industrial Chemistry, University of Genova, Via Dodecaneso 31, \\ I-16146 Genova, Italy
}

\section{TABLE OF CONTENTS}

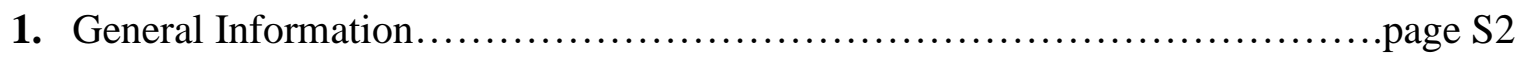

2. Experimental Procedures and Characterization Data .......................page S4

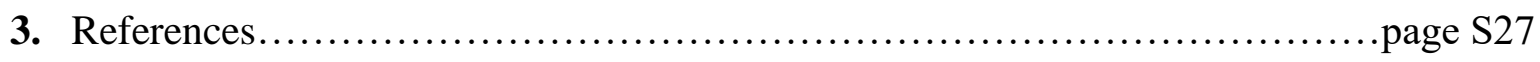

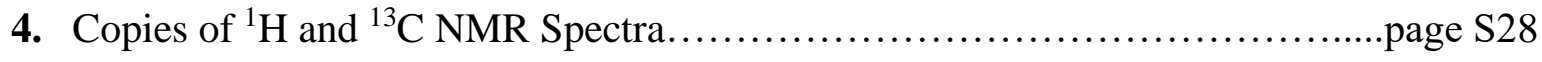




\section{General Information:}

Reagents and solvents were purchased from commercial sources and preserved under argon. More sensitive compounds were stored in a desiccator or glove-box if required. Reagents were used without further purification unless otherwise noted. All reactions were performed under argon (or nitrogen) and stirring unless otherwise noted. When needed, glassware was dried in an oven $\left(\mathrm{T}^{\circ}>100^{\circ} \mathrm{C}\right)$ or under vacuum with a heat gun $\left(\mathrm{T}^{\circ}>200^{\circ}\right)$.

When solvents are indicated as dry they were either purchased as such, distilled prior to use or were dried by a passage through a column of anhydrous alumina or copper using a Puresolv MD 5 from Innovative Technology Inc., based on the Grubbs' design. Flash column chromatography was performed using Silicycle SiliaFlash ${ }^{\circledR}$ P60 230-400 mesh or aluminium oxide. Reactions were monitored using Merck Kieselgel $60 \mathrm{~F}_{254}$ aluminium or glass backed plates. TLC's were visualized by UV fluorescence $(254 \mathrm{~nm})$ then one of the following: $\mathrm{KMnO}_{4}$, phosphomolybdic acid, ninhydrin, pancaldi, $p$-anisaldehyde, vanillin.

NMR spectra were recorded on a Brüker AvanceIII-400, Brüker Avance-400 or Brüker DPX-400 spectrometer at room temperature, ${ }^{1} \mathrm{H}$ frequency is at $400.13 \mathrm{MHz},{ }^{13} \mathrm{C}$ frequency is at $100.62 \mathrm{MHz}$. Chemical shifts $(\delta)$ are reported in parts per million $(\mathrm{ppm})$ relative to residual solvent peaks rounded to the nearest 0.01 for proton and 0.1 for carbon (ref: $\mathrm{CHCl}_{3}\left[{ }^{1} \mathrm{H}: 7.26,{ }^{13} \mathrm{C}: 77.0\right]$, Acetone $\left[{ }^{1} \mathrm{H}: 2.09,{ }^{13} \mathrm{C}: 30.60,205.87\right]$ and $\left.\mathrm{CH}_{2} \mathrm{Cl}_{2}\left[{ }^{1} \mathrm{H}: 5.30,{ }^{13} \mathrm{C}: 53.52\right]\right)$. Coupling constants $(J)$ are reported in $\mathrm{Hz}$ to the nearest $0.1 \mathrm{~Hz}$. Peak multiplicity is indicated as follows s (singlet), d (doublet), t (triplet), q (quartet), m (multiplet), p (quintet) and br (broad). Attribution of peaks was done using the multiplicities and integrals of the peaks.

IR spectra were recorded in a Jasco FT/IR-4100 spectrometer outfitted with a PIKE technology MIRacle $^{\mathrm{TM}}$ ATR accessory as neat films compressed onto a Zinc Selenide window or a Perkin Elmer Spectrum BX FT-IR. The spectra are reported in $\mathrm{cm}^{-1}$. Abbreviations used are: w (weak), m (medium), s (strong) and br (broad). 
Mass spectra were determined with a Waters ACQUITY H-class UPLC/MS ACQ-SQD by electron ionisation (EI positive and negative) or a Finnigan TSQ7000 by electrospray ionization $\left(\mathrm{ESI}^{+}\right)$. The accurate masses were done by the mass spectrometry service of the EPFL by ESI-TOF using a QTOF Ultima from Waters.

When a rigorously inert and dry atmosphere was needed the reaction was carried out in a PureLab HE 4GB 2500 Glove-box System from Innovative Technologies inc.

Melting points were measured using a Stuart SMP30 or Büchi B-540. 


\section{Experimental Procedures and Characterization Data}

\section{(isocyanomethyl)trimethylsilane ${ }^{1}$}

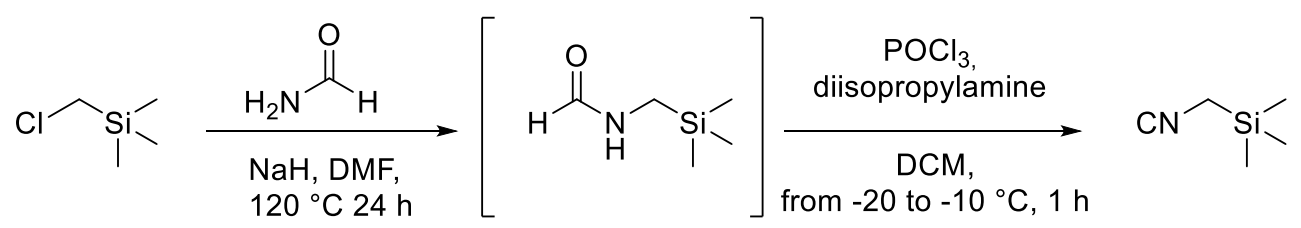

Formamide $(715 \mu \mathrm{l}, 18.0 \mathrm{mmol})$ was added to a stirred suspension of sodium hydride $(840 \mathrm{mg}$, $60 \%$ dispersion in mineral oil) in dry DMF $(18 \mathrm{~mL})$ at room temperature under nitrogen. The resulting mixture was heated at $120{ }^{\circ} \mathrm{C}$ with efficient stirring to control foaming. Around $120{ }^{\circ} \mathrm{C}$, the color of the grey suspension changed to slightly brown and stirring was continued for $45 \mathrm{~min}$. After cooling to $60^{\circ} \mathrm{C}$, chloromethyltrimethylsilane $(2.5 \mathrm{~mL}, 18 \mathrm{mmol})$ was added and the mixture was stirred at $120^{\circ} \mathrm{C}$ for 24 hours. After filtration, DMF was removed under vacuum and the crude product was used directly for the next step.

Phosphoryl chloride $(1.84 \mathrm{~mL}, 19.8 \mathrm{mmol})$ was slowly added dropwise to a solution of $\mathrm{N}$ [(trimethylsilyl)methyl]formamide $(18.0 \mathrm{mmol})$ and diisopropylamine $(6.9 \mathrm{ml}, 48.6 \mathrm{mmol})$ in DCM $(20 \mathrm{~mL})$ at $-30{ }^{\circ} \mathrm{C}$ under nitrogen atmosphere. After the addition was complete, the slightly pink suspension was stirred for an additional hour while allowing the temperature to rise to $0{ }^{\circ} \mathrm{C}$. The reaction was quenched with a solution of $\mathrm{Na}_{2} \mathrm{CO}_{3}$ (sat) $(40 \mathrm{~mL})$ and the resulting mixture was stirred at room temperature for $30 \mathrm{~min}$. The organic layer was separated and the aqueous layer was extracted with DCM $(2 \times 50 \mathrm{~mL})$. The combined organic layers were washed twice with aqueous ammonium chloride, dried over sodium sulfate and concentrated. The residue was purified by flash chromatography (PE: EtOAc 95:5) to give a yellow oil (1.301 g, 64\% yield in two steps). The crude product can be also purified by distillation: bp. $75-77^{\circ} \mathrm{C}(90$ mbar).

${ }^{1} \mathbf{H}$ NMR (400 MHz, CDCl3) $\delta 2.84(\mathrm{t}, J=2.2 \mathrm{~Hz}, 2 \mathrm{H}), 0.15(\mathrm{~s}, 9 \mathrm{H}) .{ }^{13} \mathbf{C}$ NMR (400 MHz, CDCl 3$)$ $\delta 154.4(\mathrm{t}, J=5.9 \mathrm{~Hz}), 31.4(\mathrm{t}, J=4.7 \mathrm{~Hz}),-3.6 . \mathbf{I R}: v\left(\mathrm{~cm}^{-1}\right) 2923(\mathrm{~m}), 2854(\mathrm{w}), 2361(\mathrm{w}), 2137$ (s), 1254 (w), 849 (s). HRMS (ESI) calcd for $\mathrm{C}_{5} \mathrm{H}_{12} \mathrm{NSi}^{+}[\mathrm{M}+\mathrm{H}]^{+}$114.0734; found 114.0739. 
(isocyanomethyl)triphenylphosphonium chloride $^{2}(1)$

$$
\mathrm{CN}_{\mathrm{Si}^{\prime}} \underset{\mathrm{THF}, \mathrm{rt}, 18 \mathrm{~h}}{\stackrel{\mathrm{PPh}_{3}, \mathrm{C}_{2} \mathrm{Cl}_{6}}{\longrightarrow}}\left[\mathrm{CN} \widehat{\mathrm{PPh}_{3}}\right] \mathrm{Cl}^{-}
$$

Triphenylphosphine $(1.46 \mathrm{~g}, 5.56 \mathrm{mmol})$ and $\mathrm{C}_{2} \mathrm{Cl}_{6}(1.32 \mathrm{~g}, 5.56 \mathrm{mmol})$ were weighed in a flask in glovebox and dissolved in dry THF $(40 \mathrm{~mL})$. Trimethylsilylmethyl isocyanide $(1.00 \mathrm{~g}, 8.83$ mmol), was added and the solution was stirred under inert atmosphere at room temperature for 18 h. The pale yellow precipitate was filtered off in the glovebox, washed with THF (3 x $10 \mathrm{~mL})$ and a small amount of dry and cold DCM $(2 \mathrm{~mL})$, dried under vacuum to afford a white solid (1.30 g, $70 \%$ yield based on $\left.\mathrm{PPh}_{3}\right)$. MP: $180-182.7^{\circ} \mathrm{C}$

${ }^{1}$ H NMR (400 MHz, CDCl3) $\delta 8.03(\mathrm{dd}, J=13.3,7.4 \mathrm{~Hz}, 6 \mathrm{H}), 7.84-7.80(\mathrm{~m}, 3 \mathrm{H}), 7.71(\mathrm{td}, J=$ 7.7, 3.7 Hz, 6H), $7.18(\mathrm{~d}, J=10.1 \mathrm{~Hz}, 2 \mathrm{H}) .{ }^{13} \mathbf{C}$ NMR (400 MHz, CDCl3) $\delta 164.7$ (m), 135.7 (d, $J$ $=3.2 \mathrm{~Hz}), 134.5(\mathrm{~d}, J=10.6 \mathrm{~Hz}), 130.5(\mathrm{~d}, J=13.2 \mathrm{~Hz}), 115.3(\mathrm{~d}, J=87.7 \mathrm{~Hz}), 38.2(\mathrm{~d}, J=57.6$ Hz). ${ }^{31}$ P NMR (400 MHz, CDCl 3$) \delta 21.82 . ~ I R: ~ v ~\left(\mathrm{~cm}^{-1}\right) 2973(\mathrm{w}), 2717(\mathrm{w}), 2364(\mathrm{w}), 2142(\mathrm{~m})$, 1681 (w), 1439 (s), 1115 (s). HRMS (ESI) calcd for $\mathrm{C}_{20} \mathrm{H}_{17} \mathrm{NP}^{+}[\mathrm{M}+\mathrm{H}]^{+}$302.1093; found 302.1100 .

\section{General procedure for Wittig reaction}

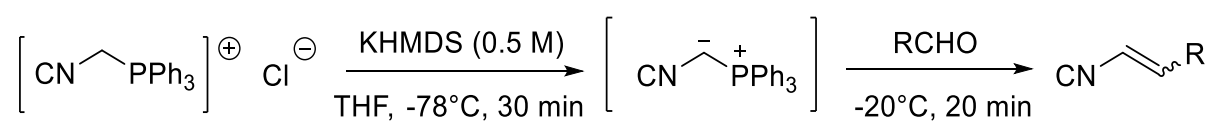

The triphenylphosphonium salt $(40.5 \mathrm{mg}, 0.12 \mathrm{mmol})$ was weighed and suspended in dry THF (1.0 $\mathrm{mL})$ under inert atmosphere in a glovebox. The resulting suspension was stirred under nitrogen atmosphere at $-78{ }^{\circ} \mathrm{C}$ and a solution of KHMDS $(0.5 \mathrm{M}$ in toluene, $240 \mu \mathrm{L})$ was slowly added. After $30 \mathrm{~min}$ the aldehyde $(0.10 \mathrm{mmol})$ was added into the suspension and the reaction mixture was stirred at $-20^{\circ} \mathrm{C}$ for 20 minutes. The reaction was quenched with water $(10 \mathrm{~mL})$ and the organic phase was extracted with ethyl acetate $(2 \times 10 \mathrm{~mL})$, dried over sodium sulfate, and concentrated. The residue was purified by column chromatography on $\mathrm{Al}_{2} \mathrm{O}_{3}$ (activity IV) to give the viniyl isocyanide. 


\section{(E)-(2-isocyanovinyl)benzene (5a)}

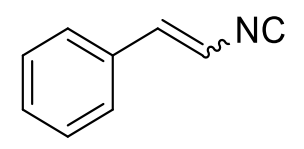

Purification: Column chromatography on $\mathrm{Al}_{2} \mathrm{O}_{3}$ (activity IV) (PE/EtOAc, 10:1) isolated as a yellow oil (9.0 mg, 70\% yield). E/Z: 1:0.4.

${ }^{1}$ H NMR (400 MHz, CDCl $) \delta 7.71(\mathrm{dd}, J=8.3,1.8 \mathrm{~Hz}, 0.8 \mathrm{H}), 7.45-7.30$ (m, 6.2H), 6.69 (dt, $J=$ 14.4, 2.4 Hz, 1H), 6.40 (dt, $J=9.9,5.1 \mathrm{~Hz}, 0.4 \mathrm{H}), 6.29$ (d, $J=14.4 \mathrm{~Hz}, 1 \mathrm{H}), 5.85$ (d, $J=9.9 \mathrm{~Hz}$, 0.4H). ${ }^{13}$ C NMR (400 MHz, CDCl3) $\delta 169.6-169.5(\mathrm{~m}), 165.2(\mathrm{t}, J=6.1 \mathrm{~Hz}), 136.7,132.7,132.5$, 132.1, 129.9, 129.7, 129.3, 129.0, 128.8, 126.7, 110.8 (t, $J=13.4 \mathrm{~Hz}), 108.8$ (t, $J=11.9 \mathrm{~Hz})$. IR:

v $\left(\mathrm{cm}^{-1}\right) 2918(\mathrm{w}), 2850(\mathrm{w}), 2363(\mathrm{w}), 2341(\mathrm{w}), 2121(\mathrm{~s}), 1653$ (w), 1449 (m). HRMS (ESI) calcd for $\mathrm{C}_{9} \mathrm{H}_{8} \mathrm{~N}^{+}[\mathrm{M}+\mathrm{H}]^{+} 130.0651$; found 130.0655 .

(E)-1-(2-isocyanovinyl)-4-nitrobenzene (5b)

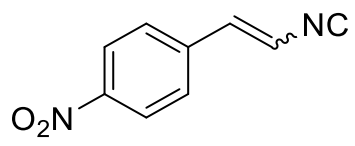

Purification: Column chromatography on $\mathrm{Al}_{2} \mathrm{O}_{3}$ (activity IV) ( $\left.\mathrm{PE}_{2} \mathrm{Et}_{2} \mathrm{O}, 9: 1\right)$ isolated as a yellow oil (14.6 mg, 85\% yield). E/Z: 1:1.

${ }^{1}$ H NMR (400 MHz, CDCl3) $\delta 8.29(\mathrm{~d}, J=8.8 \mathrm{~Hz}, 2 \mathrm{H}), 8.24(\mathrm{~d}, J=8.7 \mathrm{~Hz}, 2 \mathrm{H}), 7.86(\mathrm{~d}, J=8.8$ $\mathrm{Hz}, 2 \mathrm{H}), 7.52(\mathrm{~d}, J=8.7 \mathrm{~Hz}, 2 \mathrm{H}), 7.02(\mathrm{~d}, J=14.4 \mathrm{~Hz}, 1 \mathrm{H}), 6.53-6.50(\mathrm{~m}, 1 \mathrm{H}), 6.43(\mathrm{~d}, J=14.4$

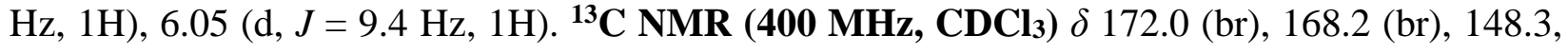
$147.9,138.8,138.4,134.4,130.0,129.8,127.4,124.3,124.0,114.3(\mathrm{t}, J=12.7 \mathrm{~Hz}), 111.2(\mathrm{t}, J=$ 11.0 Hz). IR: v (cm-1) IR 3077 (w), 2924 (w), 2853 (w), 2362 (m), 2342 (m), 2124 (s), $1596(\mathrm{w})$, 1517 (s), 1343 (s). HRMS (ESI) calcd for $\mathrm{C}_{9} \mathrm{H}_{7} \mathrm{~N}_{2} \mathrm{O}_{2}{ }^{+}[\mathrm{M}+\mathrm{H}]^{+}$175.0502; found 175.0501. 


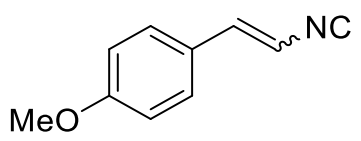

Purification: Column chromatography on $\mathrm{Al}_{2} \mathrm{O}_{3}$ (activity IV) $\left(\mathrm{PE} / \mathrm{Et}_{2} \mathrm{O}, 10: 1\right)$ isolated as a yellow oil (11.2 mg, 70\% yield). E/Z: 1:0.3.

${ }^{1}$ H NMR $\left(400 \mathrm{MHz}, \mathbf{C D C l}_{3}\right) \delta 7.68(\mathrm{~d}, J=8.8 \mathrm{~Hz}, 0.6 \mathrm{H}), 7.28(\mathrm{~d}, J=8.7 \mathrm{~Hz}, 2 \mathrm{H}), 6.95-6.87(\mathrm{~m}$, 3.6H), 6.32-6.30 (m, 0.3H), $6.17(\mathrm{~d}, J=14.4 . \mathrm{Hz}, 1 \mathrm{H}), 5.74(\mathrm{~d}, J=9.3 \mathrm{~Hz}, 0.3 \mathrm{H}), 3.85(\mathrm{~s}, 0.9 \mathrm{H})$, 3.83 (s, 3H). ${ }^{13} \mathbf{C}$ NMR (400 MHz, CDCl$) \delta 168.8$ (t, $\left.J=4.9 \mathrm{~Hz}\right), 164.3(\mathrm{t}, J=5.6 \mathrm{~Hz}), 160.9$, $160.5,136.2,131.5,131.0,128.2,125.4,125.2,114.4,114.1,108.7$ (t, $J=13.3 \mathrm{~Hz}), 106.6$ (t, $J=$ 11.2 Hz), 55.4 (2C). IR: v ( $\left.\mathrm{cm}^{-1}\right)$ IR 2923 (w), 2360 (s), 2336 (m), 2118 (m), 1653 (w), 1509 (s). HRMS (ESI) calcd for $\mathrm{C}_{10} \mathrm{H}_{10} \mathrm{NO}^{+}[\mathrm{M}+\mathrm{H}]^{+}$160.0757; found 160.0769.

\section{(E)-3-(2-isocyanovinyl)furan (5d)}

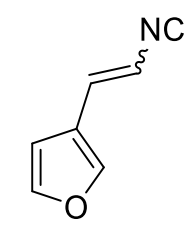

Purification: Column chromatography on $\mathrm{Al}_{2} \mathrm{O}_{3}$ (activity IV) ( $\left.\mathrm{PE} / \mathrm{Et} 2 \mathrm{O}, 9: 1\right)$ isolated as a dark yellow oil (9.5 mg, 81\% yield). E/Z: 1:0.14.

${ }^{1} \mathrm{H}$ NMR (400 MHz, CDCl 3$) \delta 7.82$ (brs, 0.14H), 7.55 (brs, 1H), 7.47 (t, $J=1.4 \mathrm{~Hz}, 0.14 \mathrm{H}$ ), 7.42 (t, $J=1.3 \mathrm{~Hz}, 1 \mathrm{H}), 6.93(\mathrm{dd}, J=2.0,0.9 \mathrm{~Hz}, 0.14 \mathrm{H}), 6.83(\mathrm{~d}, J=14.3 \mathrm{~Hz}, 1 \mathrm{H}), 6.45(\mathrm{dd}, J=1.5$, $0.8 \mathrm{~Hz}, 1 \mathrm{H}), 6.35-6.30(\mathrm{~m}, 0.14 \mathrm{H}), 6.06(\mathrm{~d}, J=14.3 \mathrm{~Hz}, 1 \mathrm{H}), 5.72(\mathrm{~d}, J=8.9 \mathrm{~Hz}, 0.14 \mathrm{H}) .{ }^{13} \mathrm{C}$ NMR (400 MHz, CDCl3) (for the major isomer $E$ ) $\delta 164.8(\mathrm{t}, J=6.2 \mathrm{~Hz}$ ), 144.5, 142.8, 126.9, 120.0, 110.2 (t, $J=13.4 \mathrm{~Hz})$, 106.6. IR: v $\left(\mathrm{cm}^{-1}\right) 2922$ (w), 2853 (w), 2361 (w), 2332 (w), 2120 (m), 1637 (w), 1509 (w), 1023 (s). HRMS (ESI) calcd for $\mathrm{C}_{7} \mathrm{H}_{6} \mathrm{NO}^{+}[\mathrm{M}+\mathrm{H}]^{+}$120.0444; found 120.0448 . 


\section{(E)-3-(2-isocyanovinyl)thiophene (5e)}

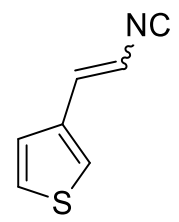

Purification: Column chromatography on $\mathrm{Al}_{2} \mathrm{O}_{3}$ (activity IV) ( $\left.\mathrm{PE} / \mathrm{Et}_{2} \mathrm{O}, 8: 2\right)$ isolated as a dark red oil (9.7 mg, 72\% yield). E/Z: 1:0.15.

${ }^{1}$ H NMR (400 MHz, CDCl3) $\delta 8.02$ (s, 0.15H), 7.76 (brs, 0.15H), 7.55-7.53 (m, 0.15H), 7.34 (s, 2H), 7.14-7.12 (m, 1H), 6.95 (d, $J=14.3 \mathrm{~Hz}, 1 \mathrm{H}), 6.48-6.43(\mathrm{~m}, 0.15 \mathrm{H}), 6.18$ (d, J = 14.4 Hz, 1H), $5.76(\mathrm{~d}, J=9.1 \mathrm{~Hz}, 0.15 \mathrm{H}) .{ }^{13} \mathrm{C}$ NMR (400 MHz, CDCl$) ~ \delta 165.2(\mathrm{t}, J=5.9 \mathrm{~Hz}), 162.5$ (br), 134.8, $134.2,130.7,128.1,127.7,127.4,126.3,126.1,126.0,124.0,110.5$ (t, $J=13.7 \mathrm{~Hz}), 107.4(\mathrm{t}, J=$ $11.8 \mathrm{~Hz})$. IR: v (cm $\left.{ }^{-1}\right) 2925(\mathrm{w}), 2854(\mathrm{w}), 2360$ (s), 2332 (s), 2126 (m), 1653 (m), 1508 (m). HRMS (ESI) calcd for $\mathrm{C}_{7} \mathrm{H}_{6} \mathrm{NS}^{+}[\mathrm{M}+\mathrm{H}]^{+}$136.0215; found 136.0213.

\section{(E)-2-(2-isocyanovinyl)furan (5f)}

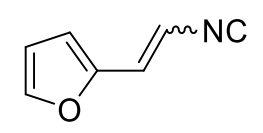

Purification: Column chromatography on $\mathrm{Al}_{2} \mathrm{O}_{3}$ (activity IV) ( $\left.\mathrm{PE} / \mathrm{Et}_{2} \mathrm{O}, 8: 2\right)$ isolated as a red oil (8.1 mg, 69\% yield). E/Z: 1:0.3.

${ }^{1}$ H NMR (400 MHz, CDCl3) $\delta 7.50(\mathrm{~d}, J=1.8 \mathrm{~Hz}, 0.3 \mathrm{H}), 7.41(\mathrm{~s}, 1 \mathrm{H}), 7.02(\mathrm{~d}, J=3.5 \mathrm{~Hz}, 0.3 \mathrm{H})$, $6.71(\mathrm{~d}, J=14.4 \mathrm{~Hz}, 1 \mathrm{H}), 6.53(\mathrm{dd}, J=3.7,1.8 \mathrm{~Hz}, 0.3 \mathrm{H}), 6.47-6.43(\mathrm{~m}, 2 \mathrm{H}), 6.43-6.39$ (m, 0.3H), $6.24(\mathrm{~d}, J=14.4 \mathrm{~Hz}, 1 \mathrm{H}), 5.68(\mathrm{~d}, J=9.1 \mathrm{~Hz}, 0.3 \mathrm{H}) .{ }^{13} \mathbf{C} \mathbf{~ N M R}\left(400 \mathrm{MHz}, \mathbf{C D C l}_{3}\right)$ (for the major isomer $E$ ) $\delta 164.3(\mathrm{t}, J=7.0 \mathrm{~Hz}), 148.3,144.2,124.4,113.3,112.2,108.7(\mathrm{t}, J=13.4 \mathrm{~Hz}) . \mathbf{I R :} v$ $\left(\mathrm{cm}^{-1}\right) 2934(\mathrm{~m}), 2853(\mathrm{~m}), 2361$ (s), $2332(\mathrm{~m}), 2121(\mathrm{w}), 1734$ (m), 1458 (m). HRMS (ESI) calcd for $\mathrm{C}_{7} \mathrm{H}_{6} \mathrm{NO}^{+}[\mathrm{M}+\mathrm{H}]^{+}$120.0444; found 120.0444 . 


\section{(E)-2-(2-isocyanovinyl)-1H-pyrrole (5g)}

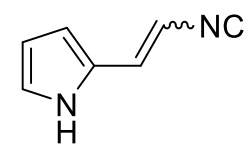

Purification: Column chromatography on $\mathrm{Al}_{2} \mathrm{O}_{3}$ (activity IV) ( $\left.\mathrm{PE} / \mathrm{Et}_{2} \mathrm{O}, 8: 2\right)$ isolated as an orange oil (8.9 mg, 75\% yield). E/Z: 1:0.15.

${ }^{1}$ H NMR (400 MHz, CDCl3) $\delta 8.26$ (brs, 1H), 7.12 (brs, 0.15H), 7.00-6.98 (m, 0.15H), 6.88-6.86 (m, 1H), 6.80 (d, J = 14.3 Hz, 1H), 6.52 (brs, 0.15H), 6.42-6.40 (m, 1H), 6.36-6.28 (m, 0.3H), 6.26 $(\mathrm{q}, J=2.6 \mathrm{~Hz}, 1 \mathrm{H}), 5.95(\mathrm{~d}, J=14.3 \mathrm{~Hz}, 1 \mathrm{H}), 5.54(\mathrm{~d}, J=8.9 \mathrm{~Hz}, 0.15 \mathrm{H}) .{ }^{13} \mathbf{C}$ NMR (400 MHz,

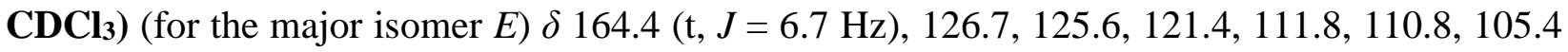
(t, J = 13.4 Hz). IR: v ( $\left.\mathrm{cm}^{-1}\right) 2961$ (w), 2924 (w), 2361 (m), 2336 (w), 2114 (s), 1459 (m), 1260 (m). HRMS (ESI) calcd for $\mathrm{C}_{7} \mathrm{H}_{7} \mathrm{~N}_{2}{ }^{+}[\mathrm{M}+\mathrm{H}]^{+} 119.0604$; found 119.0602.

\section{(E)-3-(2-isocyanovinyl)-1H-indole (5h)}

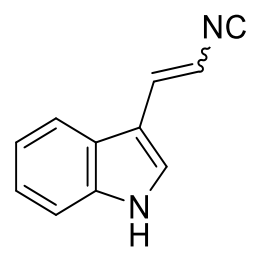

Purification: Column chromatography on $\mathrm{Al}_{2} \mathrm{O}_{3}$ (activity IV) ( $\mathrm{PE} / \mathrm{Et}_{2} \mathrm{O}, 7: 3$ ) isolated as a yellow oil (11.0 mg, 65\% yield). E/Z: 0.7:1.

${ }^{1}$ H NMR (400 MHz, CDCl 3$) \delta 8.61$ (brs, 1H), 8.41 (s, 0.7H), 8.14 (d, J = 2.6 Hz, 1H), 7.68 (d, $J$ $=7.8 \mathrm{~Hz}, 1.7 \mathrm{H}), 7.45-7.41(\mathrm{~m}, 1.7 \mathrm{H}), 7.34(\mathrm{~d}, J=2.5 \mathrm{~Hz}, 0.7 \mathrm{H}), 7.31-7.26(\mathrm{~m}, 2 \mathrm{H}), 7.25-7.21$ $(\mathrm{m}, 1.4 \mathrm{H}), 7.14(\mathrm{~d}, J=14.4 \mathrm{~Hz}, 0.7 \mathrm{H}), 6.81-6.79(\mathrm{~m}, 1 \mathrm{H}), 6.36(\mathrm{~d}, J=14.4 \mathrm{~Hz}, 0.7 \mathrm{H}), 5.75(\mathrm{~d}$, $J=8.8 \mathrm{~Hz}, 1 \mathrm{H}) .{ }^{13} \mathbf{C}$ NMR (400 MHz, CDCl3) $\delta 168.4(\mathrm{br}), 162.8(\mathrm{t}, J=5.1 \mathrm{~Hz}), 136.8,135.0$, 130.2, 126.7, 126.4, 126.3, 124.6, 124.2, 123.4, 123.2, 121.4, 120.9, 119.9, 118.0, 111.8, 111.5, 111.0, 110.1, $107.1(\mathrm{t}, J=13.0 \mathrm{~Hz}), 104.4(\mathrm{t}, J=11.4 \mathrm{~Hz})$. IR: v $\left(\mathrm{cm}^{-1}\right) 3280(\mathrm{w}), 2925(\mathrm{w}), 2854$ (w), 2361 (s), 2341 (s), 2121 (m), 1524 (m), 1459 (m). HRMS (ESI) calcd for $\mathrm{C}_{11} \mathrm{H}_{9} \mathrm{~N}_{2}{ }^{+}[\mathrm{M}+\mathrm{H}]^{+}$169.0760; found 169.0762. 
(E)-2-chloro-3-(2-isocyanovinyl)-1H-indole (5i)

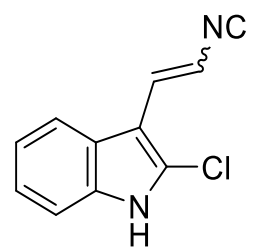

Purification: Column chromatography on $\mathrm{Al}_{2} \mathrm{O}_{3}$ (activity IV) ( $\mathrm{PE} / \mathrm{Et}_{2} \mathrm{O}, 7: 3$ ) isolated as a yellow oil (13.7 mg, 68\% yield). E/Z: 1:0.3.

${ }^{1}$ H NMR (400 MHz, CDCl3) $\delta 8.38(\mathrm{~s}, 1 \mathrm{H}), 7.75(\mathrm{~d}, J=7.0 \mathrm{~Hz}, 0.6 \mathrm{H}), 7.59(\mathrm{~d}, J=7.5 \mathrm{~Hz}, 1 \mathrm{H})$, 7.34-7.22 (m, 5.4H), 7.12 (d, $J=14.4 \mathrm{~Hz}, 1 \mathrm{H}), 6.63-6.60$ (m, 0.6H), 6.46 (d, J=14.4 Hz, 1H), 5.92 $(\mathrm{d}, J=8.9 \mathrm{~Hz}, 0.6 \mathrm{H}) .{ }^{13} \mathrm{C}$ NMR (400 MHz, CDCl3) $\delta 167.5$ (br), 163.9 (br), 134.5, 134.3, 127.7, $125.4,125.3,124.7,124.5,123.6,123.3,122.0,121.2,120.7,119.1,110.1,109.7$ (t, $J=10.6 \mathrm{~Hz})$, $108.5(\mathrm{t}, J=11.4 \mathrm{~Hz}), 106.9,106.8$. IR: $v\left(\mathrm{~cm}^{-1}\right) 3213(\mathrm{w}), 2363(\mathrm{w}), 2120(\mathrm{w}), 1627(\mathrm{~m}), 1428$ (s), 741 (s). HRMS (ESI) calcd for $\mathrm{C}_{11} \mathrm{H}_{8} \mathrm{ClN}_{2}{ }^{+}[\mathrm{M}+\mathrm{H}]^{+}$203.0371; found 203.0369.

((1E,3E)-4-isocyanobuta-1,3-dien-1-yl)benzene (5j)<smiles>CN=CC=Cc1ccccc1</smiles>

Purification: Column chromatography on $\mathrm{Al}_{2} \mathrm{O}_{3}$ (activity IV) ( $\left.\mathrm{PE} / \mathrm{Et}_{2} \mathrm{O}, 10: 1\right)$ isolated as a yellow oil (9.3 mg, 60\% yield). E/Z: 1:0.3.

${ }^{1}$ H NMR (400 MHz, CDCl $) \delta$ 7.52-7.49 (m, 0.6H), 7.43-7.28 (m, 6.2H), 7.17 (ddd, J = 15.7, 11.0, $1.0 \mathrm{~Hz}, 0.3 \mathrm{H}), 6.83-6.66(\mathrm{~m}, 3 \mathrm{H}), 6.38-6.33(\mathrm{~m}, 0.3 \mathrm{H}), 5.91(\mathrm{~d}, J=12.6 \mathrm{~Hz}, 1 \mathrm{H}), 5.66(\mathrm{~d}, J=8.1$ $\mathrm{Hz}, 0.3 \mathrm{H}) .{ }^{13} \mathbf{C}$ NMR (400 MHz, $\mathbf{C D C l}_{3}$ ) (for the major isomer $E$ ) $\delta 165.6(\mathrm{t}, J=5.9 \mathrm{~Hz}), 138.2$, 136.9, 135.8, 129.2, 128.9, 126.9, 123.0, $112.5(\mathrm{t}, J=12.3 \mathrm{~Hz})$. IR: v (cm $\left.{ }^{-1}\right) 2926(\mathrm{w}), 2361(\mathrm{~s})$, $2315(\mathrm{~m}), 2116(\mathrm{~m}), 1653(\mathrm{w}), 1508(\mathrm{~m})$. HRMS (ESI) calcd for $\mathrm{C}_{11} \mathrm{H}_{10} \mathrm{~N}^{+}[\mathrm{M}+\mathrm{H}]^{+} 156.0808$; found 156.0804 . 


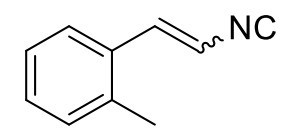

Purification: Column chromatography on $\mathrm{Al}_{2} \mathrm{O}_{3}$ (activity IV) ( $\mathrm{PE} / \mathrm{Et}_{2} \mathrm{O}$, 9:1) isolated as a brown oil (11.2 mg, 78\% yield). E/Z: 0.5:1.

${ }^{1} \mathbf{H}$ NMR (400 MHz, CDCl 3$) \delta$ 7.80-7.77 (m, 1H), $7.33(\mathrm{~d}, J=7.6 \mathrm{~Hz}, 0.5 \mathrm{H}), 7.30-7.16(\mathrm{~m}, 5 \mathrm{H})$, $6.63(\mathrm{dt}, J=9.3,4.6 \mathrm{~Hz}, 1 \mathrm{H}), 6.18(\mathrm{~d}, J=14.2 \mathrm{~Hz}, 0.5 \mathrm{H}), 5.92(\mathrm{~d}, J=9.3 \mathrm{~Hz}, 1 \mathrm{H}), 2.36(\mathrm{~s}, 1.5 \mathrm{H})$, 2.32 (s, 3H). ${ }^{13} \mathbf{C}$ NMR (400 MHz, CDCl $) \delta 168.4$ (t, $J=5.4$ Hz), $164.7(\mathrm{t}, J=6.0 \mathrm{~Hz}), 136.7$, 136.5, 134.8, 131.6, 131.3, 131.1, 130.8, 130.3, 129.7, 129.4, 128.5, 126.4, 126.1, 125.5, 111.5 (t, $J=13.0 \mathrm{~Hz}), 110.1$ (t, $J=11.8 \mathrm{~Hz}), 19.7(2 \mathrm{C}) . \mathbf{I R}: v\left(\mathrm{~cm}^{-1}\right) 2960(\mathrm{w}), 2911(\mathrm{w}), 2360(\mathrm{~m}), 2327$ (w), 2114 (s), 1699 (w), 1459 (m), 1259 (s). HRMS (ESI) calcd for $\mathrm{C}_{10} \mathrm{H}_{10} \mathrm{~N}^{+}[\mathrm{M}+\mathrm{H}]^{+}$144.0808; found 144.0818 .

(Z)-1-bromo-2-(2-isocyanovinyl)benzene (5l)

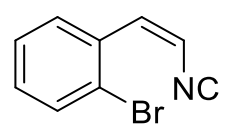

Purification: Column chromatography on $\mathrm{Al}_{2} \mathrm{O}_{3}$ (activity IV) ( $\mathrm{PE} / \mathrm{Et}_{2} \mathrm{O}$, 9:1) isolated as a yellow oil (83.2 mg, $0.40 \mathrm{mmol}, 80 \%$ yield). ${ }^{1} \mathbf{H}$ NMR (400 $\left.\mathbf{~ M H z}, \mathbf{C D C l}_{3}\right) \delta 7.92(\mathrm{dd}, J=7.8,1.7 \mathrm{~Hz}$, $1 \mathrm{H}), 7.64(\mathrm{dd}, J=7.8,1.3 \mathrm{~Hz}, 1 \mathrm{H}), 7.40(\mathrm{t}, J=7.6 \mathrm{~Hz}, 1 \mathrm{H}), 7.24(\mathrm{t}, J=7.6 \mathrm{~Hz}, 1 \mathrm{H}), 6.78-6.76(\mathrm{~m}$, 1H), $5.97(\mathrm{~d}, J=9.3 \mathrm{~Hz}, 1 \mathrm{H}) .{ }^{13} \mathbf{C}$ NMR (400 MHz, $\left.\mathbf{C D C l}_{3}\right) \delta 168.5(\mathrm{br}), 132.0,131.2,130.5$, 129.8, 129.1, 126.6, 123.1, 110.0 (t, $J=12.2 \mathrm{~Hz})$. IR: $v\left(\mathrm{~cm}^{-1}\right) 2925$ (w), 2854 (w), 2361 (s), 2124 (m), 1542 (w), 1260 (w). HRMS (ESI) calcd for $\mathrm{C}_{9} \mathrm{H}_{7} \mathrm{BrN}^{+}[\mathrm{M}+\mathrm{H}]^{+}$207.9756; found 207.9776. 
(Z)-1-(2-isocyanovinyl)-2-nitrobenzene (5m)

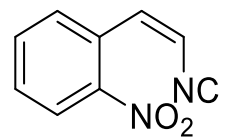

Purification: Column chromatography on $\mathrm{Al}_{2} \mathrm{O}_{3}$ (activity IV) ( $\left.\mathrm{PE} / \mathrm{Et}_{2} \mathrm{O}, 8: 2\right)$ isolated as a yellow oil (13.2 mg, $78 \%$ yield). ${ }^{1} \mathbf{H}$ NMR (400 MHz, $\left.\mathbf{C D C l}_{3}\right) \delta 8.18$ (dd, $\left.J=8.2,1.3 \mathrm{~Hz}, 1 \mathrm{H}\right), 7.83-7.81$ (m, 1H), $7.73(\mathrm{td}, J=7.6,1.3 \mathrm{~Hz}, 1 \mathrm{H}), 7.60-7.56$ (m, 1H), 7.04-7.01 (m 1H), 6.06 (d, $J=9.2 \mathrm{~Hz})$. ${ }^{13}$ C NMR (400 MHz, CDCl3) $\delta 169.7$ (br), 147.3, 133.9, 131.2, 130.1, 129.8, 127.8, 125.1, 112.3 (t, $J=12.5$ Hz). IR: v (cm $\left.{ }^{-1}\right) 2922(w), 2853(w), 2361$ (s), 2342 (s), 2120 (m), 1522 (s), 1019 (m). HRMS (ESI) calcd for $\mathrm{C}_{9} \mathrm{H}_{7} \mathrm{~N}_{2} \mathrm{O}_{2}{ }^{+}[\mathrm{M}+\mathrm{H}]^{+}$175.0502; found 175.0503 .

\section{(Z)-1-(2-isocyanovinyl)-2-methoxybenzene (5n)}

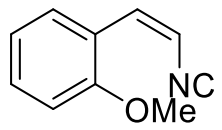

Purification: Column chromatography on $\mathrm{Al}_{2} \mathrm{O}_{3}$ (activity IV) ( $\left.\mathrm{PE}_{2} \mathrm{Et}_{2} \mathrm{O}, 9: 1\right)$ isolated as a brown oil (12.4 mg, 78\% yield). E/Z: $0.06: 1$

${ }^{1}$ H NMR (400 MHz, CDCl $) \delta 7.98(\mathrm{dd}, J=7.8,1.7 \mathrm{~Hz}, 1 \mathrm{H}), 7.38-7.33(\mathrm{~m}, 1 \mathrm{H}), 7.33-731(\mathrm{~m}$, $0.06 \mathrm{H}), 7.30-7.27(\mathrm{~m}, 0.06 \mathrm{H}), 7.10(\mathrm{~d}, J=14.7 \mathrm{~Hz}, 0.06 \mathrm{H}), 7.03(\mathrm{t}, J=7.4 \mathrm{~Hz}, 1 \mathrm{H}), 6.96(\mathrm{dd}, J=$ $7.5,1.0 \mathrm{~Hz}, 0.06 \mathrm{H}), 6.91(\mathrm{~d}, J=8.4 \mathrm{~Hz}, 1 \mathrm{H}), 6.83(\mathrm{dt}, J=9.7,4.6 \mathrm{~Hz}, 1 \mathrm{H}), 6.53(\mathrm{~d}, J=14.4 \mathrm{~Hz}$, 0.06H), $5.85(\mathrm{~d}, J=9.5 \mathrm{~Hz}, 1 \mathrm{H}), 3.98(\mathrm{~s}, 0.18 \mathrm{H}), 3.85(\mathrm{~s}, 3 \mathrm{H}) \cdot{ }^{13} \mathbf{C}$ NMR (400 MHz, CDCl3) for the major isomer (Z) $\delta 168.6$ (br), 157.1, 130.9, 129.3, 126.9, 121.5, 120.6, 110.6, 108.8 (t, $J=$ 11.9 Hz), 55.5. IR: v (cm-1) 2935 (w), 2363 (w), 2112 (w), 1599 (m), 1463 (s), 1249 (s). HRMS (ESI) calcd for $\mathrm{C}_{10} \mathrm{H}_{10} \mathrm{NO}^{+}[\mathrm{M}+\mathrm{H}]^{+} 160.0757$; found 160.0761 .

\section{(Z)-1-(2-isocyanovinyl)-2-(trifluoromethyl)benzene (5o)}




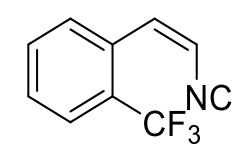

Purification: Column chromatography on $\mathrm{Al}_{2} \mathrm{O}_{3}$ (activity IV) $\left(\mathrm{PE} / \mathrm{Et}_{2} \mathrm{O}, 10: 1\right)$ isolated as a orange oil (16.3 mg, 83\% yield). ${ }^{1} \mathbf{H}$ NMR (400 MHz, $\left.\mathbf{C D C l}_{3}\right) \delta 7.91$ (d, $\left.J=7.8 \mathrm{~Hz}, 1 \mathrm{H}\right), 7.73$ (d, $J=7.9$ $\mathrm{Hz}, 1 \mathrm{H}), 7.63(\mathrm{t}, J=7.7 \mathrm{~Hz}, 1 \mathrm{H}), 7.49(\mathrm{t}, J=7.7 \mathrm{~Hz}, 1 \mathrm{H}), 6.85-6.81(\mathrm{~m}, 1 \mathrm{H}), 6.01(\mathrm{~d}, J=9.3 \mathrm{~Hz}$, 1H). ${ }^{13}$ C NMR (400 MHz, CDCl $) \delta 169.6$ (br), 132.0, 130.5, 130.3, 129.2, 128.5 (q, $J=30.2 \mathrm{~Hz}$ ), $126.1(\mathrm{q}, J=5.5 \mathrm{~Hz}), 123.8(\mathrm{q}, J=273.6 \mathrm{~Hz}), 122.4,112.2(\mathrm{t}, J=12.1 \mathrm{~Hz}) . \mathbf{I R}: \boldsymbol{v}\left(\mathbf{c m}^{-1}\right) 2363(\mathrm{w})$, 2115 (m), 1573 (w), 1456 (w), 1314 (s), 1164 (s), 1109 (s), 1036 (s). HRMS (ESI) calcd for $\mathrm{C}_{10} \mathrm{H}_{7} \mathrm{~F}_{3} \mathrm{~N}^{+}[\mathrm{M}+\mathrm{H}]^{+}$198.0525; found 198.0534 .

(Z)-2-bromo-1-(2-isocyanovinyl)-4-methylbenzene (5p)

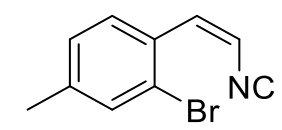

Purification: Column chromatography on $\mathrm{Al}_{2} \mathrm{O}_{3}$ (activity IV) ( $\mathrm{PE} / \mathrm{Et} t_{2} \mathrm{O}$, 9:1) isolated as a yellow foam (17.6 mg, $80 \%$ yield). ${ }^{1} \mathbf{H}$ NMR (400 MHz, $\left.\mathbf{C D C l}_{3}\right) \delta 7.84(\mathrm{~d}, J=8.0 \mathrm{~Hz}, 1 \mathrm{H}), 7.47$ (s, 1H), $7.20(\mathrm{~d}, J=8.0 \mathrm{~Hz}, 1 \mathrm{H}), 6.75-6.72(\mathrm{~m}, 1 \mathrm{H}), 5.92(\mathrm{~d}, J=9.3 \mathrm{~Hz}, 1 \mathrm{H}), 2.36(\mathrm{~s}, 3 \mathrm{H}) .{ }^{13} \mathbf{C}$ NMR $(400$ MHz, CDCl 3 ) $\delta 169.3$ (br), 141.5, 133.4, 131.3, 129.7, 129.3, 128.4, 124.1, 110.3 (t, $J=11.4 \mathrm{~Hz}$ ), 21.0. IR: $v\left(\mathrm{~cm}^{-1}\right) 2920$ (w), 2851 (w), 2358 (m), 2332 (m), 2109 (m), 1653 (w), $1458(\mathrm{~m}), 1027$ (w). HRMS (ESI) calcd for $\mathrm{C}_{10} \mathrm{H}_{9} \mathrm{BrN}^{+}[\mathrm{M}+\mathrm{H}]^{+} 221.9913$; found 221.9916 .

\section{(Z)-1-bromo-2-(2-isocyanovinyl)-4,5-dimethoxybenzene (5q)}

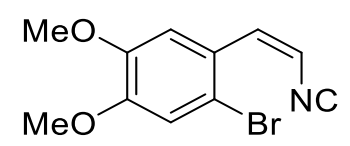

Purification: Column chromatography on $\mathrm{Al}_{2} \mathrm{O}_{3}$ (activity IV) ( $\mathrm{PE} / \mathrm{Et} t_{2} \mathrm{O}$, 9:1) isolated as a yellow oil (20.2 mg, 76\% yield). ${ }^{1} \mathbf{H}$ NMR (400 MHz, CDCl3) $\delta 7.62$ (s, 1H), 7.08 (s, 1H), 6.72 (brd, $J=$ 
$9.4 \mathrm{~Hz}, 1 \mathrm{H}), 5.87(\mathrm{~d}, J=9.4 \mathrm{~Hz}, 1 \mathrm{H}), 3.93(\mathrm{~s}, 3 \mathrm{H}), 3.91(\mathrm{~s}, 3 \mathrm{H}) .{ }^{13} \mathbf{C ~ N M R}\left(400 \mathrm{MHz}, \mathbf{C D C l}_{3}\right) \delta$ 169.6 (bs), 150.4, 148.2, 130.9, 124.3, 115.8, 115.4, 111.9, 109.0 (t, $J=11.2 \mathrm{~Hz}), 56.2$ (2C). IR: v ( $\left.\mathbf{c m}^{-1}\right) 3092$ (w), 2837 (w), 2363 (w), 2105 (m), 1597 (m), 1505 (s), 1436 (m), 1027 (s). HRMS (ESI) calcd for $\mathrm{C}_{11} \mathrm{H}_{11} \mathrm{BrNO}_{2}{ }^{+}[\mathrm{M}+\mathrm{H}]^{+}$267.9968; found 267.9978 .

\section{(Z)-4-bromo-3-(2-isocyanovinyl)-1H-indole (5r)}<smiles>C/C=C\c1c[nH]c2cccc(Br)c12</smiles>

Purification: Column chromatography on $\mathrm{Al}_{2} \mathrm{O}_{3}$ (activity IV) ( $\mathrm{PE} / \mathrm{Et} t_{2} \mathrm{O}, 7: 3$ ) isolated as a yellow oil (17.2 mg, 70\% yield).

${ }^{1} \mathbf{H}$ NMR (400 MHz, CDCl$) \delta 8.63$ (brs, 1H), $8.22(\mathrm{~d}, J=2.9 \mathrm{~Hz}, 1 \mathrm{H}), 7.74-7.72(\mathrm{~m}, 1 \mathrm{H}), 7.40-$ $7.37(\mathrm{~m}, 2 \mathrm{H}), 7.09(\mathrm{t}, J=7.9 \mathrm{~Hz}, 1 \mathrm{H}), 5.78(\mathrm{~d}, J=9.2 \mathrm{~Hz}, 1 \mathrm{H}) .{ }^{13} \mathbf{C} \mathbf{N M R}\left(400 \mathrm{MHz}, \mathbf{C D C l}_{3}\right) \delta$ $168.2(\mathrm{t}, J=5.8 \mathrm{~Hz}), 136.5,127.8,125.9,124.9,124.1,123.8,114.0,111.0,110.7,104.3$ (t, $J=$ $11.2 \mathrm{~Hz})$. IR: v (cm-1) 3276 (w), 2924 (w), 2361 (s), 2341 (s), 2123 (w), 1521 (m), 1069 (m). HRMS (ESI) calcd for $\mathrm{C}_{11} \mathrm{H}_{8} \mathrm{BrN}_{2}{ }^{+}[\mathrm{M}+\mathrm{H}]^{+} 246.9865$; found 246.9871 .

\section{(E)-2-(2-isocyanovinyl)-1,3-dimethylbenzene (5s)}

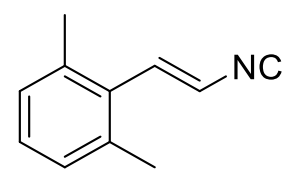

Purification: Column chromatography on $\mathrm{Al}_{2} \mathrm{O}_{3}$ (activity IV) ( $\mathrm{PE} / \mathrm{Et}_{2} \mathrm{O}$, 9:1) isolated as a yellow oil (12.0 mg, 77\% yield).

${ }^{1} \mathbf{H}$ NMR (400 MHz, CDCl$) \delta 7.14(\mathrm{dd}, J=8.5,6.5 \mathrm{~Hz}, 1 \mathrm{H}), 7.07-7.02(\mathrm{~m}, 3 \mathrm{H}), 5.90(\mathrm{dt}, J=14.7$, $1.9 \mathrm{~Hz}, 1 \mathrm{H}), 2.31(\mathrm{~s}, 6 \mathrm{H}) .{ }^{13} \mathbf{C} \mathbf{~ N M R}\left(\mathbf{4 0 0} \mathbf{~ M H z}, \mathbf{C D C l}_{3}\right) \delta 164.5(\mathrm{t}, J=5.6 \mathrm{~Hz}), 136.4,135.0,131.5$, 128.5, 128.2, 115.3 (t, $J=12.6 \mathrm{~Hz}), 21.0$. IR: $v\left(\mathrm{~cm}^{-1}\right) 2928(\mathrm{w}), 2853(\mathrm{w}), 2361(\mathrm{~m}), 2343(\mathrm{~m})$, $2108(\mathrm{~m}), 1512(\mathrm{~m}), 1211(\mathrm{~m})$. HRMS (ESI) calcd for $\mathrm{C}_{11} \mathrm{H}_{12} \mathrm{~N}^{+}[\mathrm{M}+\mathrm{H}]^{+}$158.0964; found 158.0969. 


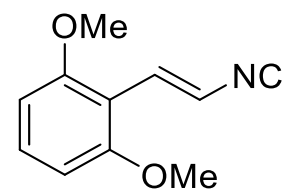

Purification: Column chromatography on $\mathrm{Al}_{2} \mathrm{O}_{3}$ (activity IV) ( $\mathrm{PE} / \mathrm{Et} t_{2} \mathrm{O}$, 9:1) isolated as a yellow foam $(16.3 \mathrm{mg}, 86 \%$ yield).

${ }^{1}$ H NMR (400 MHz, CDCl3) $\delta 7.34(\mathrm{~d}, J=14.6 \mathrm{~Hz}, 1 \mathrm{H}), 7.24(\mathrm{t}, J=8.4 \mathrm{~Hz}, 1 \mathrm{H}), 6.81(\mathrm{~d}, J=14.6$ $\mathrm{Hz}, 1 \mathrm{H}), 6.55$ (d, $J=8.4 \mathrm{~Hz}, 2 \mathrm{H}), 3.87$ (s, 6H). ${ }^{13} \mathbf{C}$ NMR (400 MHz, CDCl$) \delta 162.8$ (br), 158.9, 130.5, 127.8, 113.4 (t, $J=12.0 \mathrm{~Hz}), 110.5,103.7,55.8$. IR: $v\left(\mathrm{~cm}^{-1}\right) 2840(\mathrm{w}), 2121(\mathrm{w}), 1596$ (m), 1477 (m), $1256(\mathrm{~m}), 1113$ (s). HRMS (ESI) calcd for $\mathrm{C}_{11} \mathrm{H}_{12} \mathrm{NO}_{2}{ }^{+}[\mathrm{M}+\mathrm{H}]^{+}$190.0863; found 190.0862 .

(E)-1,3-dichloro-2-(2-isocyanovinyl)benzene (5u)<smiles>[Na]/N=C/c1c(Cl)cccc1Cl</smiles>

Purification: Column chromatography on $\mathrm{Al}_{2} \mathrm{O}_{3}$ (activity IV) ( $\left.\mathrm{PE} / \mathrm{Et}_{2} \mathrm{O}, 9: 1\right)$ isolated as a dark yellow oil (16.1 mg, $82 \%$ yield).

${ }^{1} \mathbf{H}$ NMR $\left(400 \mathrm{MHz}, \mathbf{C D C l}_{3}\right) \delta 7.36(\mathrm{~d}, J=8.1 \mathrm{~Hz}, 2 \mathrm{H}), 7.22-7.18(\mathrm{~m}, 1 \mathrm{H}), 7.07(\mathrm{dt}, J=14.8,2.9$ $\mathrm{Hz}, 1 \mathrm{H}), 6.51(\mathrm{~d}, J=14.8 \mathrm{~Hz}) .{ }^{13} \mathrm{C}$ NMR $\left(400 \mathrm{MHz}, \mathbf{C D C l}_{3}\right) \delta 167.1(\mathrm{t}, J=5.8 \mathrm{~Hz}), 134.7,130.5$, 130.0, 129.7, 128.9, $118.2(\mathrm{t}, J=12.7 \mathrm{~Hz})$. IR: $v\left(\mathrm{~cm}^{-1}\right) 2911(\mathrm{w}), 2363(\mathrm{~s}), 2343(\mathrm{~s}), 2121(\mathrm{~m})$, 1559 (m). HRMS (ESI) calcd for $\mathrm{C}_{9} \mathrm{H}_{6} \mathrm{Cl}_{2} \mathrm{~N}^{+}[\mathrm{M}+\mathrm{H}]^{+}$197.9872; found 197.9870 .

(E)-(4-isocyanobut-3-en-1-yl)benzene (5v)<smiles>CN=CCCCc1ccccc1</smiles> 
Purification: Column chromatography on $\mathrm{Al}_{2} \mathrm{O}_{3}$ (activity IV) ( $\left.\mathrm{PE} / \mathrm{Et}_{2} \mathrm{O}, 9: 1\right)$. Yield: $45 \%$, isolated as a yellow oil (7.1 mg, $45 \%$ yield). E/Z: 1:0.35.

${ }^{1}$ H NMR (400 MHz, CDCl $) \delta$ 7.32-7.29 (m, 3H), 7.24-7.19 (m, 2.1H), 7.16-7.14 (m, 2H), 6.19$6.11(\mathrm{~m}, 1 \mathrm{H}), 5.76-5.71(\mathrm{~m}, 0.35 \mathrm{H}), 5.68(\mathrm{~d}, J=14.1 \mathrm{~Hz}, 1 \mathrm{H}), 2.78-2.71(\mathrm{~m}, 2.7 \mathrm{H}), 2.67-2.61(\mathrm{~m}$, $0.7 \mathrm{H}), 2.43-2.37(\mathrm{~m}, 2 \mathrm{H}) .{ }^{13} \mathbf{C} \mathbf{~ N M R}\left(400 \mathbf{~ M H z}, \mathbf{C D C l}_{3}\right)$ (for the major isomer $\left.E\right) \delta 162.1(\mathrm{t}, \mathrm{J}=$ 6.5 Hz), 140.1, 137.9, 128.6, 128.3, 126.4, 112.9 (t, $J=12.7 \mathrm{~Hz}), 34.6,31.3$. IR: $v\left(\mathrm{~cm}^{-1}\right) 2923(\mathrm{w})$, 2853 (w), 2361 (s), 2323 (w), 2118 (s), 1508 (m), 1458 (s), 1270 (m). HRMS (ESI) calcd for $\mathrm{C}_{11} \mathrm{H}_{12} \mathrm{~N}^{+}[\mathrm{M}+\mathrm{H}]^{+}$158.0964; found 158.0972.

\section{(E)-(((6-isocyanohex-5-en-1-yl)oxy)methyl)benzene (5w)}

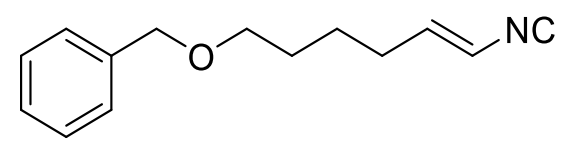

Purification: Column chromatography on $\mathrm{Al}_{2} \mathrm{O}_{3}$ (activity IV) (PE/Et $\left.2 \mathrm{O}, 10: 1\right)$ isolated as a yellow oil (14.4 mg, 67\% yield).

${ }^{1} \mathrm{H}$ NMR (400 MHz, CDCl $) \delta$ 7.35-7.30 (m, 5H), 6.15-6.10 (m, 1H), $5.67(\mathrm{~d}, J=14.4 \mathrm{~Hz}, 1 \mathrm{H})$, 4.50 (s, 2H), 3.49-3.45 (m, 2H), 2.12-2.06 (m, 2H), 1.67-1.58 (m, 2H), $1.54-1.50(\mathrm{~m}, 2 \mathrm{H}) .{ }^{13} \mathrm{C}$ NMR (400 MHz, CDCl $) \delta 161.8(\mathrm{t}, J=6.5 \mathrm{~Hz}), 138.8,138.4,137.0,128.4,127.6,112.5(\mathrm{t}, J=$ 12.8 Hz), 73.0, 69.7, 29.3, 29.1, 25.1. IR: v ( $\left.\mathrm{cm}^{-1}\right) 2924$ (w), 2854 (w), 2363 (m), 2342 (w), 2120 (m), 1457 (m), 1260 (m), 1099 (s), 1028 (s). HRMS (ESI) calcd for $\mathrm{C}_{14} \mathrm{H}_{18} \mathrm{NO}^{+}[\mathrm{M}+\mathrm{H}]^{+}$216.1383; found 216.1392 .

(E)-2-(5-isocyanopent-4-en-1-yl)isoindoline-1,3-dione (5x)

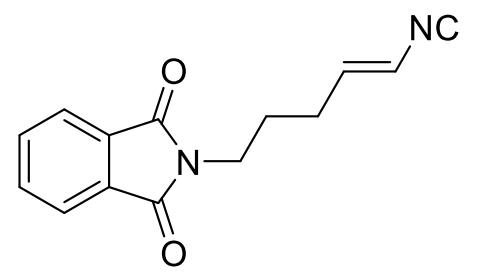


Purification: Column chromatography on $\mathrm{Al}_{2} \mathrm{O}_{3}$ (activity IV) $\left(\mathrm{PE} / \mathrm{Et}_{2} \mathrm{O}, 8: 2\right)$ isolated as a yellow oil (13.9 mg, 58\% yield).

${ }^{1} \mathbf{H}$ NMR (400 MHz, CDCl $) \delta 7.85(\mathrm{dd}, J=5.4,3.1 \mathrm{~Hz}, 2 \mathrm{H}), 7.73(\mathrm{dd}, J=5.5,3.0 \mathrm{~Hz}, 2 \mathrm{H}), 6.17$ $-6.09(\mathrm{~m}, 1 \mathrm{H}), 5.75(\mathrm{~d}, J=14.2 \mathrm{~Hz}, 1 \mathrm{H}), 3.70(\mathrm{t}, J=7.0 \mathrm{~Hz}, 2 \mathrm{H}), 2.14(\mathrm{qd}, J=7.4,1.6 \mathrm{~Hz}, 2 \mathrm{H})$, 1.80 - 1.79 (m, 2H). ${ }^{13} \mathbf{C}$ NMR (400 MHz, CDCl $) \delta$ 168.3, $162.3(\mathrm{t}, J=6.0 \mathrm{~Hz}), 137.2,134.0$, 131.9, 123.3, 113.2 (t, $J=12.3 \mathrm{~Hz}), 37.0,27.2$, 26.9. IR: v $\left(\mathrm{cm}^{-1}\right) 2942(\mathrm{w}), 2363(\mathrm{w}), 2124(\mathrm{~m})$, 1771 (w), 1705 (s), 1437 (w), 1396 (m). HRMS (ESI) calcd for $\mathrm{C}_{14} \mathrm{H}_{13} \mathrm{~N}_{2} \mathrm{O}_{2}{ }^{+}[\mathrm{M}+\mathrm{H}]^{+}$241.0972; found 241.0978. 


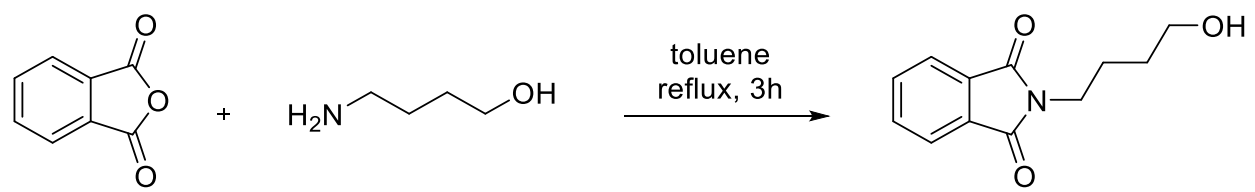

A mixture of 4-amino-1-butanol (445 mg, $5.0 \mathrm{mmol})$ and phthalic anhydride $(740 \mathrm{mg}, 5.0 \mathrm{mmol})$ in toluene $(20 \mathrm{~mL})$ was heated to reflux under Dean-Stark conditions for $3 \mathrm{~h}$. After cooling, removal of solvent under vacuum produced the crude product which was purified by flash chromatography eluting with EtOAc:PE (10:1) to afford a colorless oil, which upon standing became a white crystalline solid (1.070 g, $98 \%$ yield). MP: $49-50{ }^{\circ} \mathrm{C}$.

${ }^{1} \mathbf{H}$ NMR (400 MHz, CDCl$) \delta 7.82(\mathrm{dd}, J=5.4,3.0 \mathrm{~Hz}, 2 \mathrm{H}), 7.70(\mathrm{dd}, J=5.4,3.0 \mathrm{~Hz}, 2 \mathrm{H}), 3.72$ $(\mathrm{t}, J=7.0 \mathrm{~Hz}, 2 \mathrm{H}), 3.68(\mathrm{t}, J=6.4 \mathrm{~Hz}, 2 \mathrm{H}), 1.81-1.73(\mathrm{~m}, 3 \mathrm{H}), 1.64-1.59(\mathrm{~m}, 2 \mathrm{H}) .{ }^{13} \mathbf{C}$ NMR $(400$ MHz, CDCl 3$) \delta$ 168.5, 133.9, 132.0, 123.2, 62.3, 37.7, 29.7, 25.1. IR: $v\left(\mathrm{~cm}^{-1}\right) 3415$ (w), 2931 (w), $2364 \quad(\mathrm{w}), \quad 1679 \quad(\mathrm{~s}), 1399 \quad(\mathrm{~m}), 1042 \quad$ (s). HRMS (ESI) calcd for $\mathrm{C}_{12} \mathrm{H}_{14} \mathrm{NO}_{3}{ }^{+}[\mathrm{M}+\mathrm{H}]^{+}$220.0968; found 220.0968 .

\section{4-(1,3-dioxoisoindolin-2-yl)butanal was prepared according to the literature procedure. ${ }^{3}$}
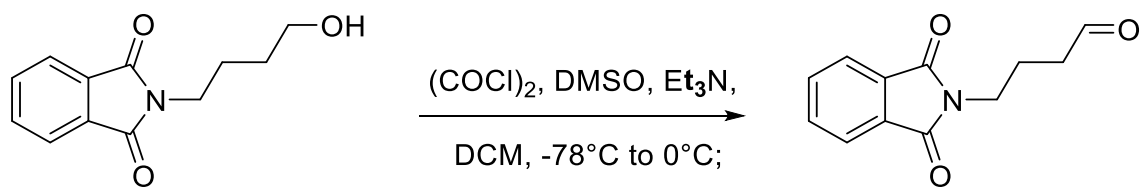

A solution of DMSO $(568 \mu \mathrm{L}, 8.0 \mathrm{mmol})$ in DCM $(4 \mathrm{~mL})$ was added over $30 \mathrm{~min}$ to a stirred solution of oxalyl chloride $(350 \mu \mathrm{L}, 4.0 \mathrm{mmol})$ in DCM $(15 \mathrm{~mL})$ at $-78{ }^{\circ} \mathrm{C}$. Upon completion of the addition, the mixture was stirred at $-78^{\circ} \mathrm{C}$ for $5 \mathrm{~min}$, followed by addition of a solution of the alcohol (440 mg, $2.0 \mathrm{mmol}$ ) in DCM (4 mL) over $30 \mathrm{~min}$ at $-78^{\circ} \mathrm{C}$ and the resulting mixture was stirred for $40 \mathrm{~min}$. Then $\mathrm{Et}_{3} \mathrm{~N}(1.67 \mathrm{~mL}, 12.0 \mathrm{mmol})$ was added dropwise over $10 \mathrm{~min}$. The resulting 
mixture was allowed to warm to $0{ }^{\circ} \mathrm{C}$ and stirred at $0{ }^{\circ} \mathrm{C}$ for $1 \mathrm{~h}$. Water $(10 \mathrm{~mL})$ was added to quench the reaction. The organic layer was then separated and further washed with water $(2 \times 15$ $\mathrm{mL}$ ) and brine. The thus obtained organic layer was dried over anhydrous sodium sulfate and concentrated. The residue was subjected to flash chromatography, eluting with PE: EtOAc (7:3), yielding a white solid (415 mg, yield 96\%). MP: $60-62{ }^{\circ} \mathrm{C}$.

${ }^{1}$ H NMR (400 MHz, CDCl $) \delta 9.76(\mathrm{t}, J=1.2 \mathrm{~Hz}, 1 \mathrm{H}), 7.83(\mathrm{dd}, J=5.5,3.1 \mathrm{~Hz}, 2 \mathrm{H}), 7.71(\mathrm{dd}, J$ $=5.5,3.1 \mathrm{~Hz}, 2 \mathrm{H}), 3.73(\mathrm{t}, J=6.8 \mathrm{~Hz}, 2 \mathrm{H}), 2.53(\mathrm{td}, J=7.3,1.2 \mathrm{~Hz}, 2 \mathrm{H}), 2.01(\mathrm{p}, J=7.0 \mathrm{~Hz}, 2 \mathrm{H})$. ${ }^{13}$ C NMR (400 MHz, CDCl3) $\delta$ 200.8, 168.3, 134.0, 132.0, 123.2, 41.1, 37.1, 21.1. IR: v (cm $\left.{ }^{-1}\right)$ 2853 (w), 2363 (w), 1703 (s), 1396 (m), 1131 (w). HRMS (ESI) calcd for $\mathrm{C}_{12} \mathrm{H}_{12} \mathrm{NO}_{3}{ }^{+}[\mathrm{M}+\mathrm{H}]^{+} 218.0812$; found 218.0813

\section{5-(benzyloxy)pentan-1-ol was prepared according to the literature procedure. ${ }^{4}$}

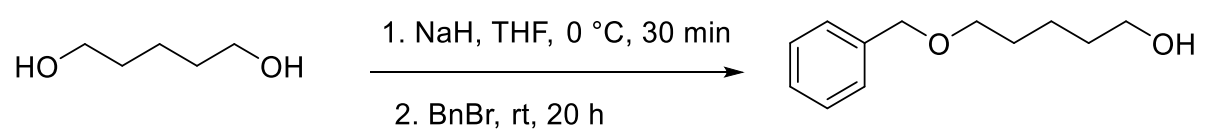

A solution of 1,5 pentanediol $(6.2 \mathrm{~mL}, 58.8 \mathrm{mmol})$ in THF $(25 \mathrm{~mL})$ was slowly added to a slurry of $\mathrm{NaH}(1.11 \mathrm{~g}$ of $60 \%$ suspension in mineral oil, $10.0 \mathrm{mmol})$ in $\mathrm{THF}(12.5 \mathrm{~mL})$ at $0{ }^{\circ} \mathrm{C}$ under nitrogen. After stirring for $30 \mathrm{~min}$ at $0{ }^{\circ} \mathrm{C}$ a solution of benzyl bromide $(1.0 \mathrm{~mL}, 8.4 \mathrm{mmol})$ in THF $(12.5 \mathrm{~mL})$ was slowly added over $10 \mathrm{~min}$. The reaction was warmed to room temperature and stirred for $20 \mathrm{~h}$. Water was added $(5 \mathrm{~mL})$ and THF was removed under reduced pressure to yield an oil that was divided between water $(100 \mathrm{~mL})$ and EtOAc $(25 \mathrm{~mL})$. The layers were separated and the aqueous phase was extracted with EtOAc $(3 \times 25 \mathrm{~mL})$. The combined organic layers were washed with water $(5 \times 10 \mathrm{~mL})$ and finally with brine, dried over sodium sulfate and concentrated to afford a colorless oil (1.59 $\mathrm{g}, 98 \%$ yield $)$.

${ }^{1}$ H NMR (400 MHz, CDCl $) \delta 7.37-7.28(\mathrm{~m}, 5 \mathrm{H}), 4.50(\mathrm{~s}, 2 \mathrm{H}), 3.64(\mathrm{t}, J=6.5 \mathrm{~Hz}, 2 \mathrm{H}), 3.48(\mathrm{t}$, $J=6.5 \mathrm{~Hz}, 2 \mathrm{H}), 1.69-1.55(\mathrm{~m}, 4 \mathrm{H}), 1.49-1.42$ (m, 3H). ${ }^{13} \mathbf{C}$ NMR (400 MHz, CDCl3) $\delta 138.5$, 128.3, 127.6, 127.5, 72.9, 70.3, 62.8, 32.5, 29.5, 22.4. IR: v(cm $\mathrm{cm}^{-1} 3341(\mathrm{w}), 2934(\mathrm{~s}), 2858(\mathrm{~s})$, 2363 (m), 1704 (w), 1364 (m), 1054 (s). HRMS (ESI) calcd for $\mathrm{C}_{12} \mathrm{H}_{18} \mathrm{O}_{2}$ [M+] 194.1307; found 194.1314. 


\section{5-(benzyloxy)pentanal}

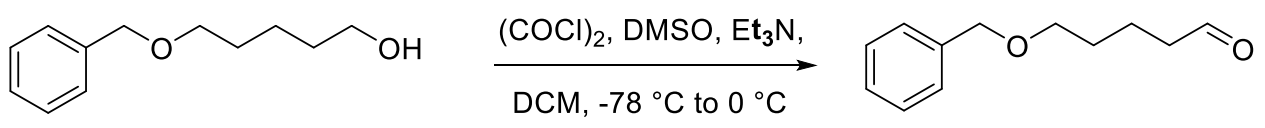

A solution of DMSO $(1.40 \mathrm{~mL}, 19.7 \mathrm{mmol})$ in DCM $(8 \mathrm{~mL})$ was added over $30 \mathrm{~min}$ to a stirred solution of oxalyl chloride $(844 \mu \mathrm{L}, 9.84 \mathrm{mmol})$ in DCM $(32 \mathrm{~mL})$ at $-78{ }^{\circ} \mathrm{C}$. Upon completion of the addition, the mixture was stirred at $-78^{\circ} \mathrm{C}$ for $5 \mathrm{~min}$, followed by addition of a solution of the alcohol $(1.590 \mathrm{~g}, 8.2 \mathrm{mmol})$ in DCM $(8 \mathrm{~mL})$ over $30 \mathrm{~min}$ at $-78{ }^{\circ} \mathrm{C}$ and the resulting mixture was stirred for $20 \mathrm{~min}$. Then $\mathrm{Et}_{3} \mathrm{~N}(4.8 \mathrm{~mL}, 34.4 \mathrm{mmol})$ was added dropwise over $10 \mathrm{~min}$. The resulting mixture was allowed to warm to $0{ }^{\circ} \mathrm{C}$ and stirred at $0{ }^{\circ} \mathrm{C}$ for $30 \mathrm{~min}$. The reaction was quenched by addition of a solution of $\mathrm{NaHCO}_{3}$ (sat) $(20 \mathrm{~mL})$. The layers were separated and the aqueous phase was extracted with DCM $(2 \times 25 \mathrm{~mL})$. The combined organic layers were washed with water ( $2 \times 10 \mathrm{~mL})$ and finally with brine, dried over sodium sulfate and concentrated. The residue was purified by flash chromatography eluting with $\mathrm{Et}_{2} \mathrm{O}: \mathrm{PE} 1: 1$ to afford a yellow oil $(1.51 \mathrm{~g}, 96 \%$ yield).

${ }^{1}$ H NMR (400 MHz, CDCl $) \delta 9.76$ (t, $\left.J=1.7 \mathrm{~Hz}, 1 \mathrm{H}\right), 7.39-7.26(\mathrm{~m}, 5 \mathrm{H}), 4.50$ (s, 2H), 3.49 (t, $J=6.1 \mathrm{~Hz}, 2 \mathrm{H}), 2.46(\mathrm{td}, J=7.2,1.7 \mathrm{~Hz}, 2 \mathrm{H}), 1.79-1.71(\mathrm{~m}, 2 \mathrm{H}), 1.69-1.62(\mathrm{~m}, 2 \mathrm{H}) .{ }^{13} \mathbf{C ~ N M R}$ (400 MHz, CDCl3) $\delta 202.5,138.4,128.4,127.6,127.6,73.0,69.7,43.6,29.1,19.0$. IR: $v\left(\mathrm{~cm}^{-1}\right)$ 2935 (w), 2856 (w), 2362 (w), 1723 (w), 1455 (w), 1360 (w), 1095 (s). HRMS (ESI) calcd for $\mathrm{C}_{12} \mathrm{H}_{16} \mathrm{O}_{2}[\mathrm{M}+]$ 192.1150; found 192.1157. 


\section{General procedure for Ugi reaction 5}

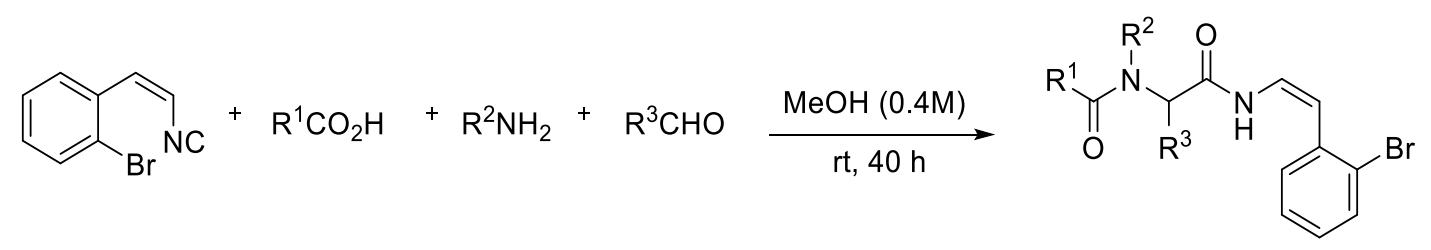

The carboxylic acid $(0.23 \mathrm{mmol})$, amine $(0.23 \mathrm{mmol})$, and aldehyde $(0.19 \mathrm{mmol})$ were dissolved in methanol $(1 \mathrm{M})$. The solution was allowed to stand for $10 \mathrm{~min}$, and then was added in one portion to a flask containing the isocyanide $(0.19 \mathrm{mmol})$. The resulting solution $(0.4 \mathrm{M})$ was allowed to stir at room temperature for $40 \mathrm{~h}$. When the reaction was complete (monitored by TLC), the solvent was removed in vacuo, and the residue was purified by flash column chromatography on silica gel.

\section{(Z)-2-(N-benzylpropionamido)-N-(2-bromostyryl)-4-phenylbutanamide (18)}

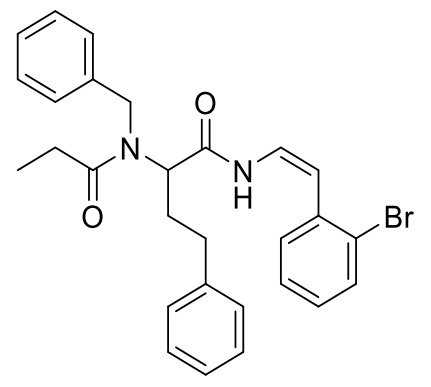

Purification: Flash chromatography (PE/EtOAc, 7:3) isolated as a yellow foam (75 mg, 79\% yield).

${ }^{1}$ H NMR (400 MHz, CDCl3) $\delta 8.63(\mathrm{~d}, J=11.5 \mathrm{~Hz}, 1 \mathrm{H}), 7.64(\mathrm{dd}, J=8.1,1.2 \mathrm{~Hz}, 1 \mathrm{H}), 7.40-7.33$ $(\mathrm{m}, 2 \mathrm{H}), 7.30-7.21(\mathrm{~m}, 5 \mathrm{H}) 7.18-714(\mathrm{~m}, 2 \mathrm{H}), 7.05(\mathrm{t}, J=6.5 \mathrm{~Hz}, 4 \mathrm{H}), 6.94(\mathrm{dd}, J=11.5,9.6 \mathrm{~Hz}$, $1 \mathrm{H}), 5.81(\mathrm{~d}, J=9.6 \mathrm{~Hz}, 1 \mathrm{H}), 4.80(\mathrm{t}, J=7.2 \mathrm{~Hz}, 1 \mathrm{H}), 4.54(\mathrm{~d}, J=17.4 \mathrm{~Hz}, 1 \mathrm{H}), 4.46(\mathrm{~d}, J=17.4$ $\mathrm{Hz}, 1 \mathrm{H}), 2.66-2.59(\mathrm{~m}, 1 \mathrm{H}), 2.55-2.48(\mathrm{~m}, 1 \mathrm{H}), 2.41-2.29(\mathrm{~m}, 3 \mathrm{H}), 1.96-1.87(\mathrm{~m}, 1 \mathrm{H}), 1.07(\mathrm{t}, J=$ $7.2 \mathrm{~Hz}, 3 \mathrm{H}) .{ }^{13} \mathbf{C}$ NMR (400 MHz, CDCl3) $\delta 176.2,168.4,140.6,136.6,135.2,133.1,129.5,128.9$, 128.6, 128.4, 128.3, 127.7, 127.6, 126.2, 126.1, 123.8, 122.5, 109.8, 57.8, 48.9, 32.6, 29.5, 27.1, 9.3. IR: v ( $\left.\mathrm{cm}^{-1}\right) 3265(\mathrm{w}), 2936(\mathrm{w}), 2364(\mathrm{w}), 1698$ (m), 1650 (s), 1496 (s), 1456 (s), 1257 (m), 1027 (m). HRMS (ESI) calcd for $\mathrm{C}_{28} \mathrm{H}_{30} \mathrm{BrN}_{2} \mathrm{O}_{2}{ }^{+}[\mathrm{M}+\mathrm{H}]^{+}$505.1485; found 505.1489. 


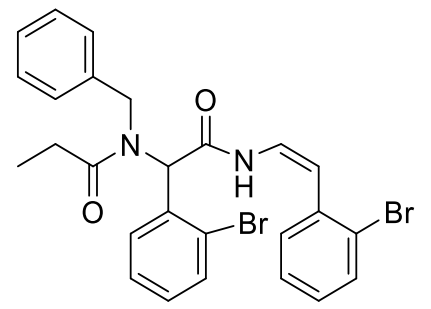

Purification: Flash chromatography (PE/EtOAc, 8:2) isolated as a pale yellow foam (73 mg, 70\% yield).

${ }^{1}$ H NMR (400 MHz, CDCl 3$) \delta$ 7.65-7.62 (m, 1H), 7.56-7.53 (m, 2H), $7.46(\mathrm{~d}, J=7.8 \mathrm{~Hz}, 1 \mathrm{H})$, 7.24-7.16 (m, 4H), 7.13-7.09 (m, 5H), 7.06-7.01 (m, 2H), $5.92(\mathrm{~s}, 1 \mathrm{H}), 5.80(\mathrm{~d}, J=9.5 \mathrm{~Hz}, 1 \mathrm{H})$, $4.65(\mathrm{~d}, J=17.3 \mathrm{~Hz}, 1 \mathrm{H}), 4.45(\mathrm{~d}, J=17.3 \mathrm{~Hz}, 1 \mathrm{H}), 2.50-2.34(\mathrm{~m}, 2 \mathrm{H}), 1.16(\mathrm{t}, J=7.4 \mathrm{~Hz}, 3 \mathrm{H})$. ${ }^{13}$ C NMR (400 MHz, CDCl3) $\delta$ 175.4, 166.4, 136.2, 135.1, 133.6, 133.3, 133.2, 131.1, 130.4, $128.9,128.5,128.4,127.9,127.5,127.4,126.5,126.2,126.1,125.9,123.8,122.6,110.1,63.8,50.8$, 27.1, 9.3. IR: $v\left(\mathrm{~cm}^{-1}\right), 3275(\mathrm{w}), 2976(\mathrm{w}), 2363$ (s), 2319 (w), 1699 (m), 1652 (s), 1508 (m), 1458 (s), 1419 (m), 1258 (m), 1026 (m). HRMS (ESI) calcd for $\mathrm{C}_{26} \mathrm{H}_{25} \mathrm{Br}_{2} \mathrm{~N}_{2} \mathrm{O}_{2}{ }^{+}[\mathrm{M}+\mathrm{H}]^{+} 555.0277$; found 555.0275.

\section{N-(1-(1H-indol-1-yl)-1-oxo-4-phenylbutan-2-yl)-N-benzylpropionamide (21)}

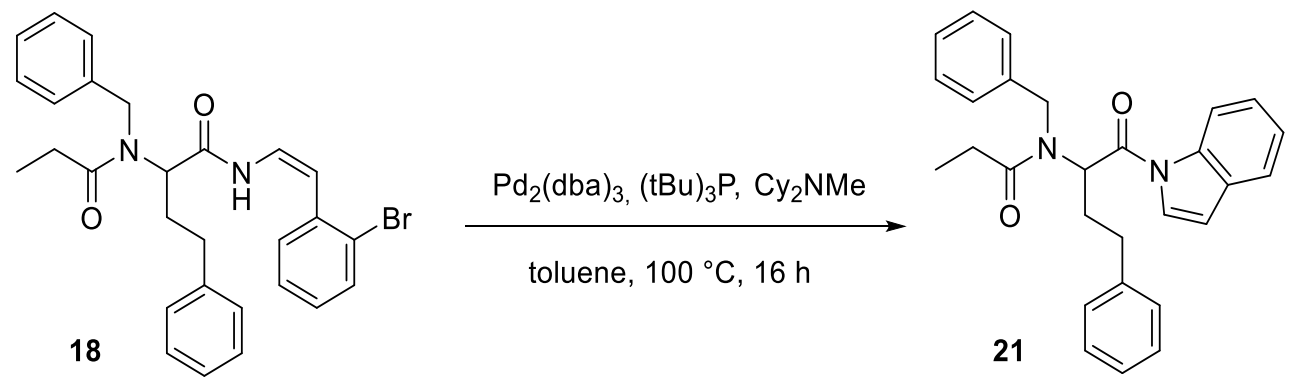

Tris(dibenzylideneacetone)dipalladium(0) $(5.0 \mathrm{mg}, 5.5 \mu \mathrm{mol}),(t \mathrm{Bu})_{3} \mathrm{P}(5.3 \mu \mathrm{L}, 0.02 \mathrm{mmol})$ and $\mathrm{Cy}_{2} \mathrm{NMe}(28 \mu \mathrm{L}, 0.13 \mathrm{mmol})$ were added sequentially to a solution of bromide $18(61.2 \mathrm{mg}, 0.11$ mmol) in toluene $(700 \mu \mathrm{L})$ under inert atmosphere in a glovebox. The resulting suspension was stirred at $100{ }^{\circ} \mathrm{C}$ for 16 hours. The reaction mixture was cooled to room temperature, quenched with water and the organic phase was extracted with ethyl acetate $(2 \times 10 \mathrm{~mL})$, the organic layers were dried over anhydrous sodium sulfate, and concentrated. The residue was purified by flash chromatography to give the product 21 as a white foam $(36.1 \mathrm{mg}, 85 \%$ yield). 
${ }^{1} \mathrm{H}$ NMR (400 MHz, CDCl$) \delta 8.20-8.18(\mathrm{~m}, 1 \mathrm{H}), 7.74(\mathrm{~d}, J=3.9 \mathrm{~Hz}, 1 \mathrm{H}), 7.53-7.51(\mathrm{~m}, 1 \mathrm{H})$, 7.29-7.15 (m, 8H), 7.00-6.97 (m, 2H), 6.90-6.88 (m, 2H), $6.62(\mathrm{~d}, J=3.8 \mathrm{~Hz}, 1 \mathrm{H}), 6.25(\mathrm{t}, J=7.3$ $\mathrm{Hz}, 1 \mathrm{H}), 4.63(\mathrm{~d}, J=17.9 \mathrm{~Hz}, 1 \mathrm{H}), 4.58(\mathrm{~d}, J=17.9 \mathrm{~Hz}, 1 \mathrm{H}), 2.78-2.71(\mathrm{~m}, 1 \mathrm{H}), 2.65-2.57(\mathrm{~m}$, 1H), 2.44-2.23 (m, 3H), 2.16-2.07 (m, 1H), 1.14 (t, $J=7.3 \mathrm{~Hz}, 3 \mathrm{H}) .{ }^{13} \mathbf{C}$ NMR (400 MHz, CDCl$)$ $\delta$ 175.0, 169.3, 140.8, 136.5, 135.5, 130.7, 128.5, 128.4, 128.3, 127.0, 126.2, 125.4, 125.3, 124.9, 124.0, 120.6, 116.6, 109.9, 54.5, 47.4, 32.2, 30.9, 27.0, 9.5. IR: $v\left(\mathrm{~cm}^{-1}\right) 2936$ (w), 2364 (m), 1702 (m), 1652 (s), 1454 (s). HRMS (ESI) calcd for $\mathrm{C}_{28} \mathrm{H}_{29} \mathrm{~N}_{2} \mathrm{O}_{2}{ }^{+}[\mathrm{M}+\mathrm{H}]^{+} 425.2224$; found 425.2234 .

\section{N-benzyl-N-(6-oxo-5,6-dihydroindolo[2,1-a]isoquinolin-5-yl)propionamide (23)}

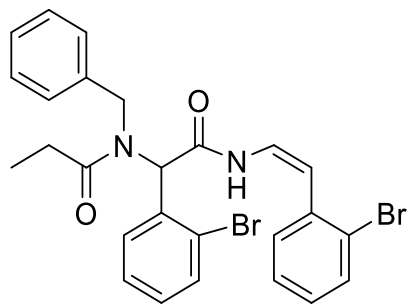

25

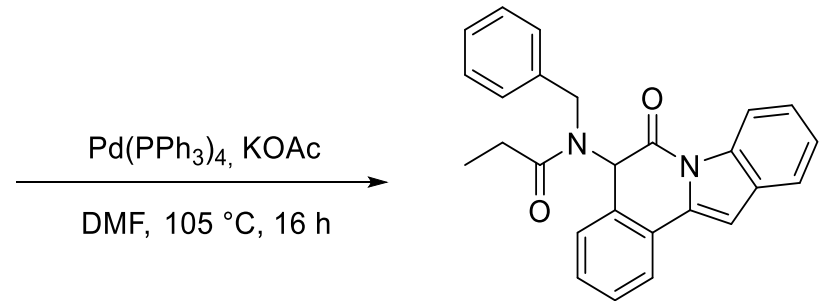

23

Potassium acetate $(24.0 \mathrm{mg}, 0.25 \mathrm{mmol})$ and tetrakis(triphenylphosphine)palladium(0) (12.0 mg, $0.01 \mathrm{mmol})$ were added sequentially to a solution of bromide 25 (56.0 $\mathrm{mg}, 0.1 \mathrm{mmol})$ in DMF (1.6 $\mathrm{mL}$ ) under inert atmosphere in a glovebox. The resulting suspension was stirred at $105{ }^{\circ} \mathrm{C}$ for 16 hours. The reaction mixture was cooled to room temperature, quenched with water and the organic phase was extracted with ethyl acetate $(2 \times 10 \mathrm{~mL})$, dried over anhydrous sodium sulfate, and concentrated. The residue was purified by flash chromatography to give the product $\mathbf{2 3}$ as a white foam (32.2 $\mathrm{mg}, 82 \%$ yield).

${ }^{1}$ H NMR (400 MHz, CDCl3) $\delta$ 8.13-8.11 (m, 1H), 7.58 (d, $J=7.9$ Hz, 1H), 7.51-7.44 (m, 3H), 7.22-7.07 (m, 8H), $6.39-6.33(\mathrm{~m}, 2 \mathrm{H}), 4.67(\mathrm{~d}, J=17.5 \mathrm{~Hz}, 1 \mathrm{H}), 4.57$ (d, J=17.5 Hz, 1H), 2.50$2.37(\mathrm{~m}, 2 \mathrm{H}), 1.15(\mathrm{t}, J=7.0 \mathrm{~Hz}, 3 \mathrm{H}) .{ }^{13} \mathbf{C}$ NMR (400 MHz, CDCl3) $\delta$ 175.9, 167.4, 136.9, 135.9, $133.5,133.4,132.9,131.3,130.5,128.4,127.9,127.5,127.1,126.6,126.2,126.0,124.5,123.1$, 112.2, 62.6, 49.8, 27.3, 9.4. IR: v (cm-1) 3284 (w), 2923 (w), 2360 (s), 1648 (s), 1464 (w). HRMS (ESI) calcd for $\mathrm{C}_{26} \mathrm{H}_{22} \mathrm{~N}_{2} \mathrm{NaO}_{2}{ }^{+}[\mathrm{M}+\mathrm{Na}]^{+}$417.1573; found 417.1575. 
methyl 2-(N-benzylpropionamido)-4-phenylbutanoate ${ }^{5}(20)$

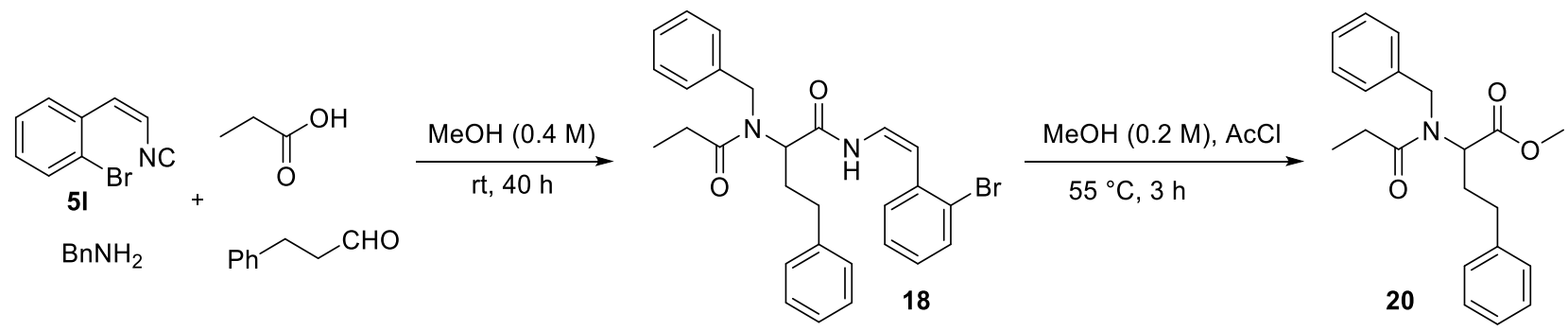

Propionic acid (17 $\mu \mathrm{L}, 0.23 \mathrm{mmol})$, benzylamine $(25 \mu \mathrm{L}, 0.23 \mathrm{mmol})$, and hydrocinnamaldehyde $(25 \mu \mathrm{L}, 0.19 \mathrm{mmol})$ were dissolved in methanol $(0.5 \mathrm{~mL})$. The solution was allowed to stand for $10 \mathrm{~min}$, and then was added in one portion to a flask containing isonitrile $\mathbf{5 l}$ (40 $\mathrm{mg}, 0.19 \mathrm{mmol})$. The resulting solution was allowed to stir at room temperature for $40 \mathrm{~h}$. When the reaction was complete (monitored by TLC) the methanol volume is doubled and acetyl chloride (135 $\mu \mathrm{L}, 1.9$ mmol) was added in one portion. The flask was heated to $55{ }^{\circ} \mathrm{C}$ for $3 \mathrm{~h}$. When TLC showed complete conversion to methyl ester, the reaction mixture was cooled to room temperature, the solvent was removed under vacuum, and the residue was taken up in $\mathrm{CH}_{2} \mathrm{Cl}_{2}$ and filtered. Purification by flash column chromatography on silica gel, eluting with PE/ EtOAc (7:3) gave compound 20 as a colorless oil (45 mg, yield $72 \%$ in two steps).
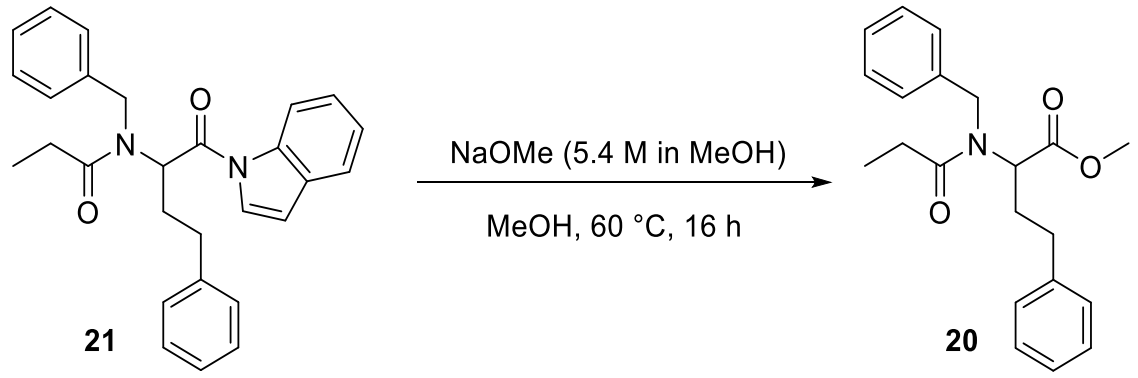

To a solution of indole 21 (30 mg, $0.07 \mathrm{mmol})$ in $\mathrm{MeOH}(1.0 \mathrm{~mL})$ at room temperature was added a solution of $\mathrm{NaOMe}$ in $\mathrm{MeOH}(5.4 \mathrm{M})(70 \mu \mathrm{L}, 0.35 \mathrm{mmol})$ and the mixture was stirred overnight at $60{ }^{\circ} \mathrm{C}$. The reaction was quenched with $\mathrm{NH}_{4} \mathrm{Cl}(10 \mathrm{~mL})$ and extracted with EtOAc $(2 \times 15 \mathrm{~mL})$. The combined organic layers were dried over anhydrous $\mathrm{Na}_{2} \mathrm{SO}_{4}$ and concentrated in vacuum. Purification by flash column chromatography on silica gel, eluting with PE: EtOAc (8:2) gave the methyl ester 20 as a colorless oil (18 mg, yield: $82 \%)$. 
${ }^{1} \mathbf{H}$ NMR (400 MHz, $\mathbf{C D C l}_{3}$ ) (two rotamers in ratio of 1:0.4) $\delta$ 7.36-7.33 (m, 2H), 7.30-7.28 (m, 2.6H), 7.25-7.21 (m, 5.4H), 7.18-7.14 (m, 1.2H), $7.04(\mathrm{~d}, J=7.2 \mathrm{~Hz}, 2 \mathrm{H}), 6.95(\mathrm{~d}, J=7.2 \mathrm{~Hz}$, $0.8 \mathrm{H}), 4.72-4.70(\mathrm{~m}, 1.4 \mathrm{H}), 4.63(\mathrm{~d}, J=17.3 \mathrm{~Hz}, 1 \mathrm{H}), 4.49$ (d, $J=15.3 \mathrm{~Hz}, 0.4 \mathrm{H}), 4.44$ (d, $J=17.3$ $\mathrm{Hz}, 1 \mathrm{H}), 4.38-4.34(\mathrm{~m}, 0.4 \mathrm{H}), 3.62$ (s, 3H), 3.48 (s, 1.2H), 2.69-2.42 (m, 3H), 2.41-2.30 (m, 3.6H), 2.17-2.10 (m, 0.4H), 2.03-1.92 (m, 1.4H), 1.17-1.12 (m, 4.2H). ${ }^{13}$ C NMR (400 MHz, CDCl3) $\delta$ 175.0, 174.9, 171.7, 171.2, 141.0, 140.1, 138.3, 137.0, 128.7, 128.5, 128.4, 128.3, 128.2, 127.6, 127.1, 126.6, 126.3, 126.0, 58.7, 57.9, 52.2, 52.0, 50.4, 46.4, 32.9, 32.0, 31.2, 29.7, 26.9, 26.6, 9.4, 9.3. IR: v ( $\left.\mathrm{cm}^{-1}\right) 2935$ (w), 2856 (w), 2364 (w), 1738 (s), 1651 (s), 1455 (m), 1216 (s). HRMS (ESI) calcd for $\mathrm{C}_{21} \mathrm{H}_{25} \mathrm{NNaO}_{3}{ }^{+}[\mathrm{M}+\mathrm{Na}]^{+}$362.1727; found 362.1737.

\section{S-ethyl 2-(N-benzylpropionamido)-4-phenylbutanethioate (19)}
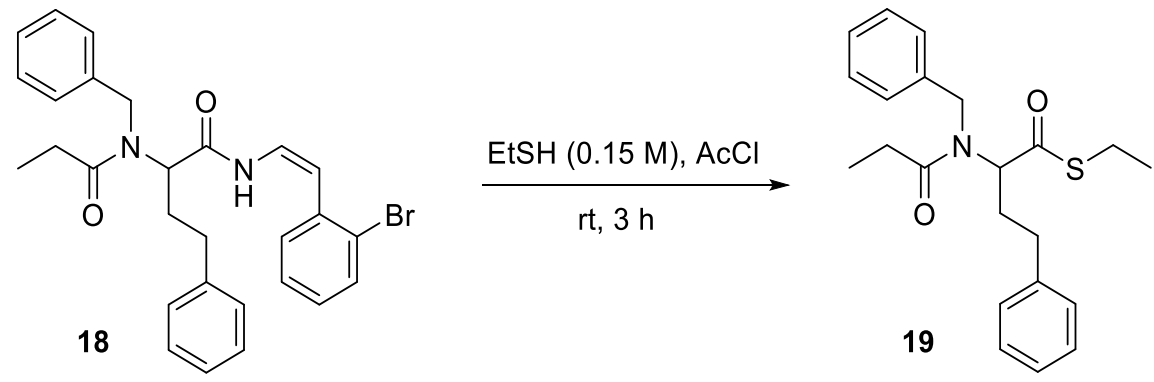

To a solution of the Ugi adduct $18(75.6 \mathrm{mg}, 0.15 \mathrm{mmol})$ in EtSH $(1.0 \mathrm{~mL})$ at room temperature was added acetyl chloride (108 $\mu \mathrm{L}, 1.5 \mathrm{mmol})$ in one portion and the solution was stirred at room temperature for $3 \mathrm{~h}$. When TLC showed complete conversion to the thioester, the reaction was quenched with $\mathrm{NaHCO}_{3}(10 \mathrm{~mL})$ and extracted with EtOAc $(3 \times 15 \mathrm{~mL})$. The combined organic layers were dried over anhydrous $\mathrm{Na}_{2} \mathrm{SO}_{4}$ and concentrated in vacuum. Purification by flash column chromatography on silica gel, eluting with PE: EtOAc (7:3) gave thioester 19 as a colorless oil (60 mg, yield: $80 \%)$.

${ }^{1}$ H NMR (400 MHz, CDCl3) (two rotamers) $\delta$ 7.36-7.28 (m, 4H), 7.22-7.14 (m, 4H), $6.98(\mathrm{~d}, J=$ $7.4 \mathrm{~Hz}, 1 \mathrm{H}), 6.83(\mathrm{~d}, J=7.3 \mathrm{~Hz}, 1 \mathrm{H}), 5.13(\mathrm{t}, J=7.5 \mathrm{~Hz}, 1 \mathrm{H}), 4.70(\mathrm{~d}, J=17.6 \mathrm{~Hz}, 1 \mathrm{H}), 4.43$ (d, $J=17.6 \mathrm{~Hz}, 1 \mathrm{H}), 2.85(\mathrm{q}, J=7.5 \mathrm{~Hz}, 2 \mathrm{H}), 2.62-2.54(\mathrm{~m}, 1 \mathrm{H}), 2.41-2.35(\mathrm{~m}, 4 \mathrm{H}), 1.90-1.82(\mathrm{~m}$, 1H), 1.26-1.15 (m, 6H). ${ }^{13} \mathbf{C}$ NMR (400 MHz, CDCl 3$) \delta 199.6,199.0,175.5,175.3,141.0,140.1$, $138.8,137.3,128.8,128.5,128.4,128.3,128.2$, 127.6, 126.4, 126.3, 126.0, 66.8, 64.1, 49.8, 33.0, 32.3, 31.3, 31.1, 27.3, 26.9, 23.7, 23.4, 14.5, 14.4, 9.5, 9.4. IR: v $\left(\mathrm{cm}^{-1}\right) 2933(\mathrm{w}), 2856(\mathrm{w}), 2364$ 
(m), 2330 (w), 1684 (s), 1660 (s), 1456 (m), 1262 (m), 1023 (m). HRMS (ESI) calcd for $\mathrm{C}_{22} \mathrm{H}_{27} \mathrm{NNaO}_{2} \mathrm{~S}^{+}[\mathrm{M}+\mathrm{Na}]^{+}$392.1655; found 392.1660.

\section{2-(N-benzylpropionamido)-4-phenylbutanoic acid ${ }^{6}$ (22)}
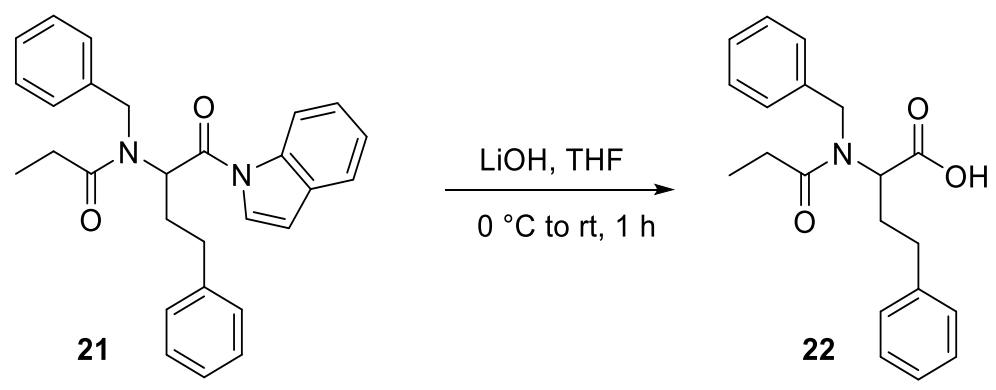

To a solution of indole $21(45.4 \mathrm{mg}, 0.1 \mathrm{mmol})$ in THF $(1.2 \mathrm{~mL})$ at $0{ }^{\circ} \mathrm{C}$ was added $1.0 \mathrm{M} \mathrm{LiOH}$ (aq) $(230 \mu \mathrm{L}, 0.2 \mathrm{mmol})$ and the mixture was warmed to room temperature. After completion of the reaction (60 min, monitored by TLC), the reaction mixture was basified with an aqueous solution of $\mathrm{NaOH}(1 \mathrm{M}, 10 \mathrm{~mL})$. The aqueous phase was washed with EtOAc $(3 \times 10 \mathrm{~mL})$. The combined organic layers were back-extracted with $1.0 \mathrm{M} \mathrm{NaOH}(2 \times 15 \mathrm{~mL})$. The combined aqueous phase was carefully acidified to $\mathrm{pH}=2$ using $6 \mathrm{M} \mathrm{HCl}$, and extracted with diethyl ether $(6 \times 10 \mathrm{~mL})$. The combined organic layers were dried over anhydrous $\mathrm{Na}_{2} \mathrm{SO}_{4}$ and concentrated under vacuum to give acid 22 as a yellow oil (27.5 $\mathrm{mg}, 85 \%$ yield).

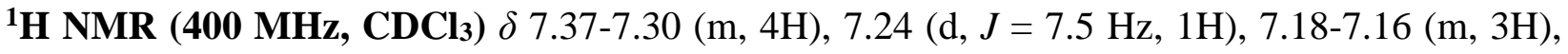
$7.05(\mathrm{~d}, J=7.2 \mathrm{~Hz}, 2 \mathrm{H}), 4.61(\mathrm{~d}, J=16.8 \mathrm{~Hz}, 2 \mathrm{H}), 4.38(\mathrm{~d}, J=16.8 \mathrm{~Hz}, 1 \mathrm{H}), 4.20(\mathrm{t}, J=6.9 \mathrm{~Hz}$, $1 \mathrm{H}), 2.71-2.64(\mathrm{~m}, 1 \mathrm{H}), 2.60-2.49(\mathrm{~m}, 1 \mathrm{H}), 2.42(\mathrm{q}, J=7.3 \mathrm{~Hz}, 2 \mathrm{H}), 2.35-2.29(\mathrm{~m}, 1 \mathrm{H}), 2.09-2.00$ $(\mathrm{m}, 1 \mathrm{H}), 1.16(\mathrm{t}, J=7.3 \mathrm{~Hz}, 3 \mathrm{H}) .{ }^{13} \mathbf{C}$ NMR (400 MHz, CDCl$) \delta$ 176.7, 173.5, 140.5, 135.8, 129.0, 128.5, 128.3, 128.0, 126.8, 126.2, 61.2, 52.5, 32.7, 30.7, 9.2. IR: v ( $\left.\mathrm{cm}^{-1}\right) 3026(\mathrm{w}), 2924$ (s), 2343 (w), 1733 (s), 1601 (s), 1455 (s). HRMS (ESI) calcd for $\mathrm{C}_{20} \mathrm{H}_{23} \mathrm{NNaO}_{3}{ }^{+}[\mathrm{M}+\mathrm{Na}]^{+}$348.1570; found 348.1580 . 


\section{References}

1. A. C. Brouwer, A. M. Van Leuse, Synth. Commun. 1986, 16, 865-869.

2. G. Zinner, W. P. Fehlhammer, Angew. Chem. Int. Ed. 1985, 24, 979-980.

3. X. Xiao, S. Antony, G. Kohlhagen, Y. Pommierb, M. Cushmana, Bioorg. Med. Chem. 2004, 12, 5147-5160.

4. I. S. Young, M. A. Kerr, J. Am. Chem. Soc. 2007, 129, 1465-1469.

5. T. A. Keating, R. W. Armstrong, J. Am. Chem. Soc. 1996, 118, 2574-2583.

6. M. Rubinshtein, C. R. James, J. L. Young, Y. J. Ma, Y. Kobayashi, N. C. Gianneschi, J. Yang, Org. Lett. 2010, 12, 3560-3563. 


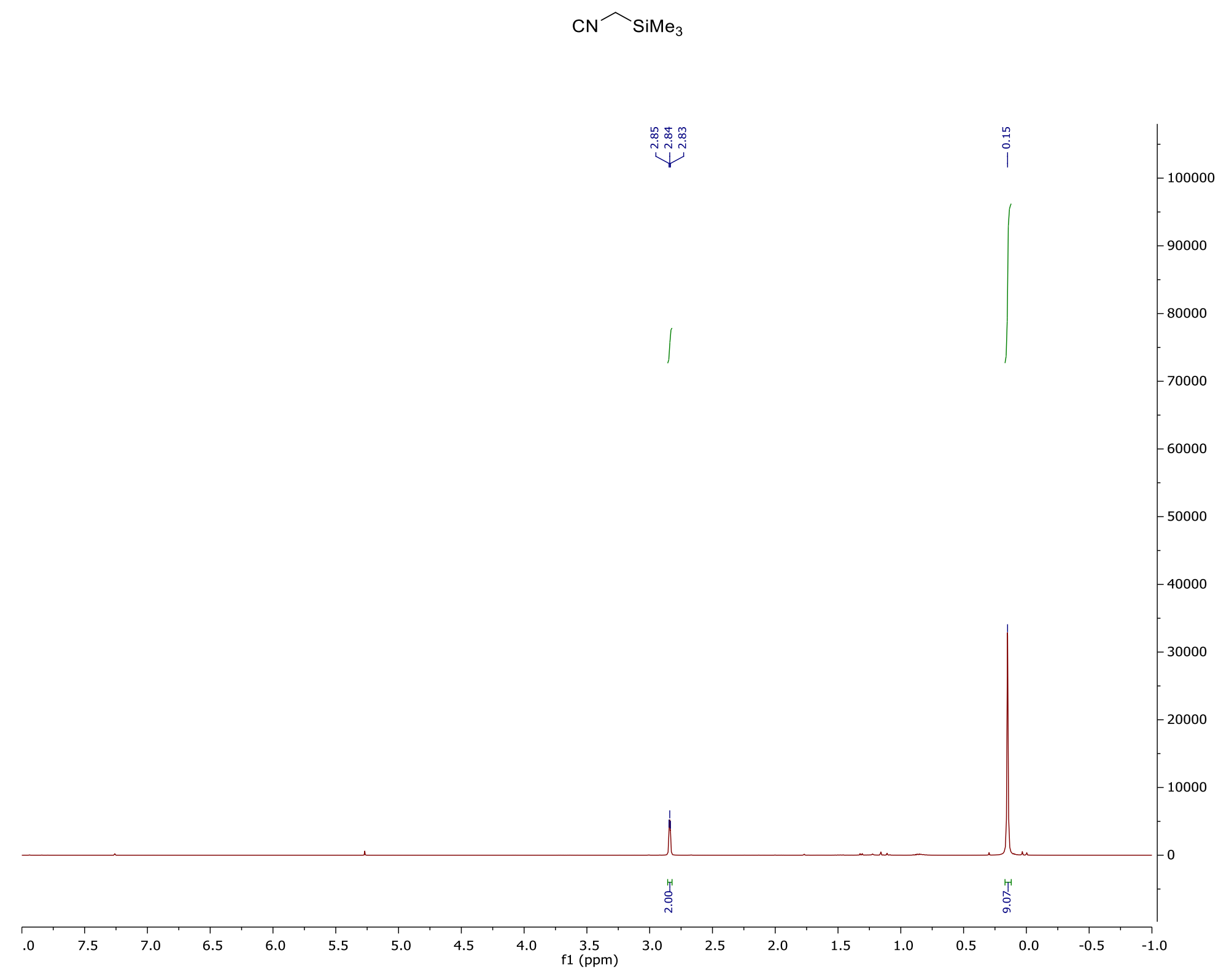




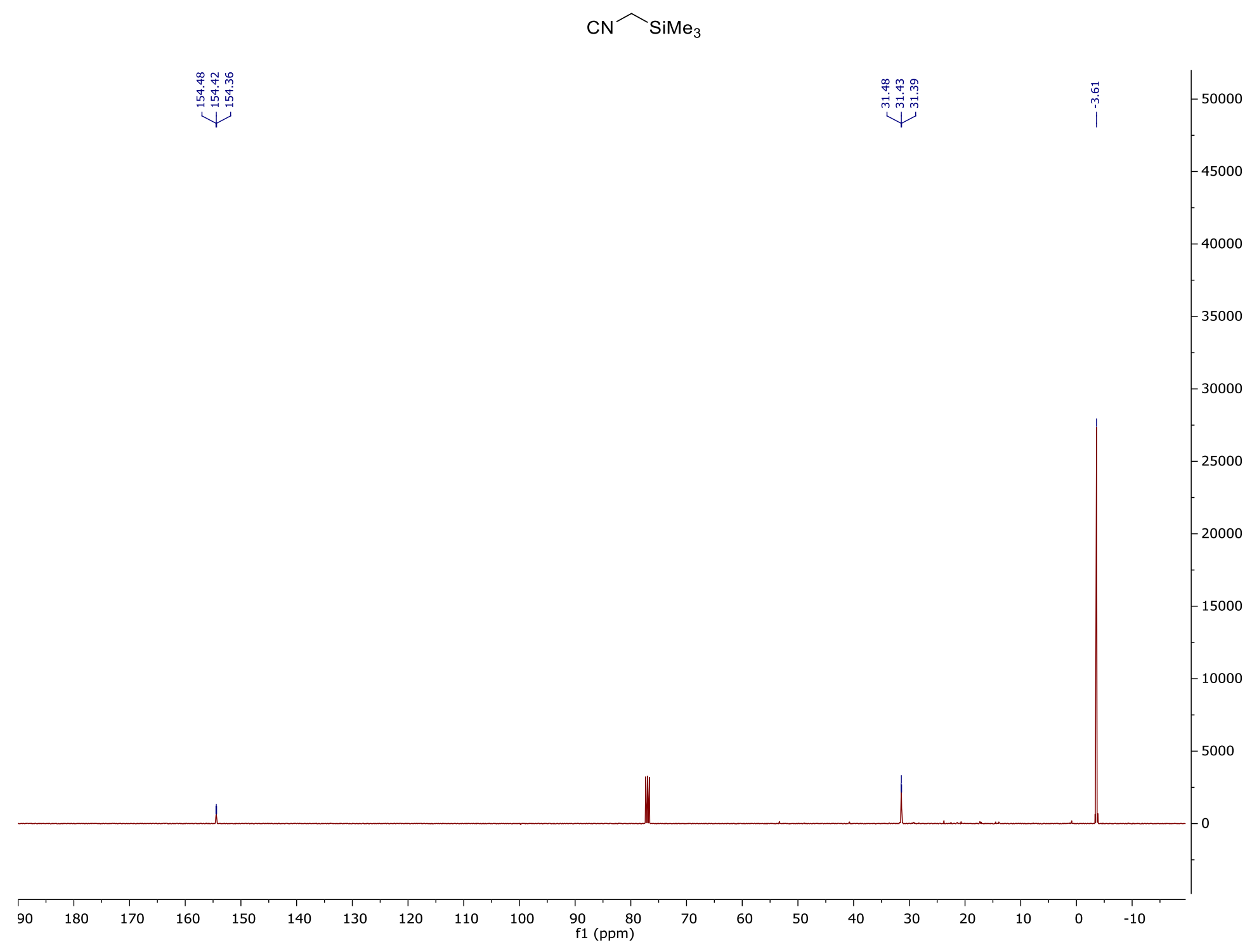




$$
\left[\underset{1}{\mathrm{CN}} \underset{\mathrm{PPh}_{3}}{\sim} \mathrm{Cl}^{-}\right.
$$

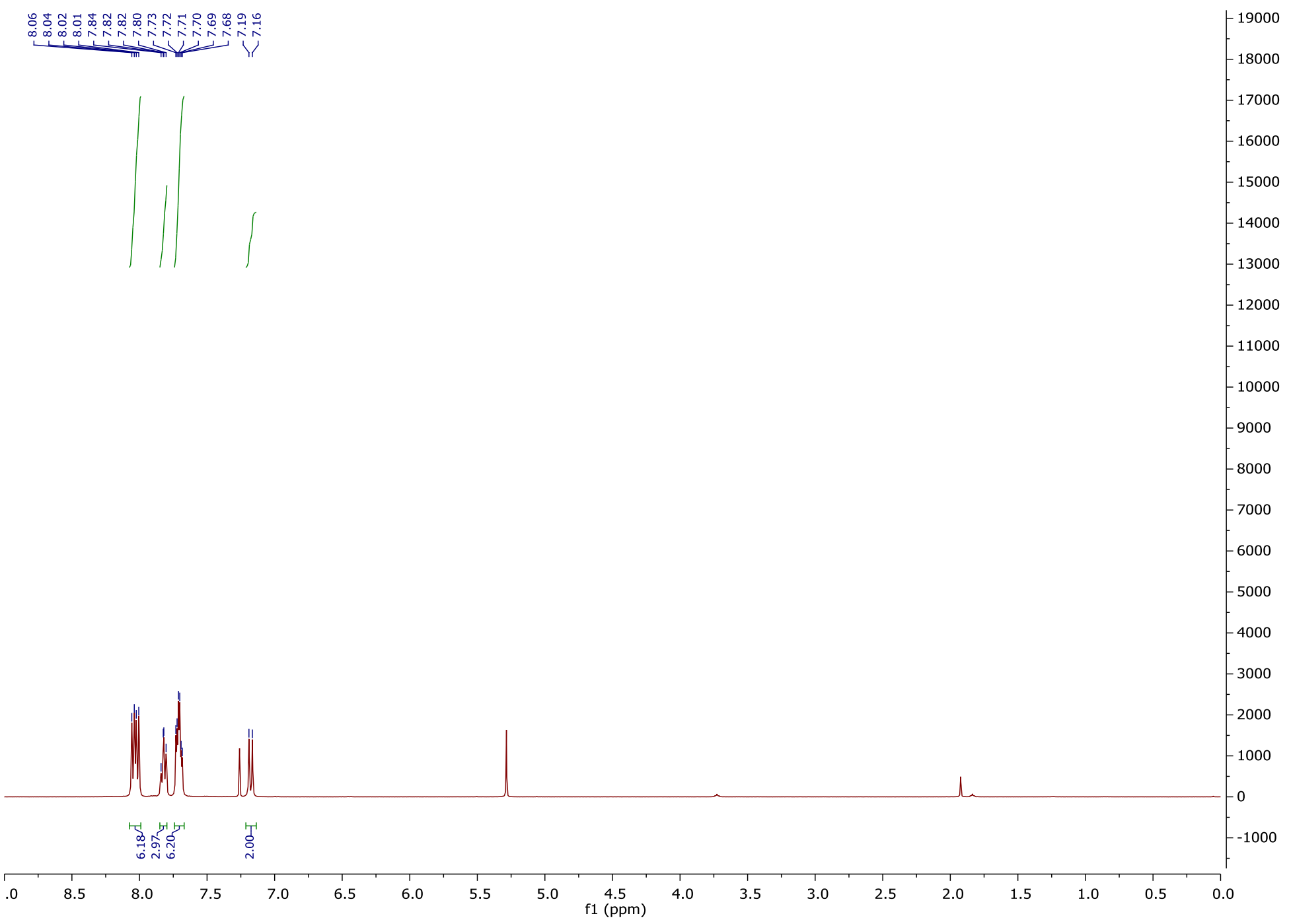




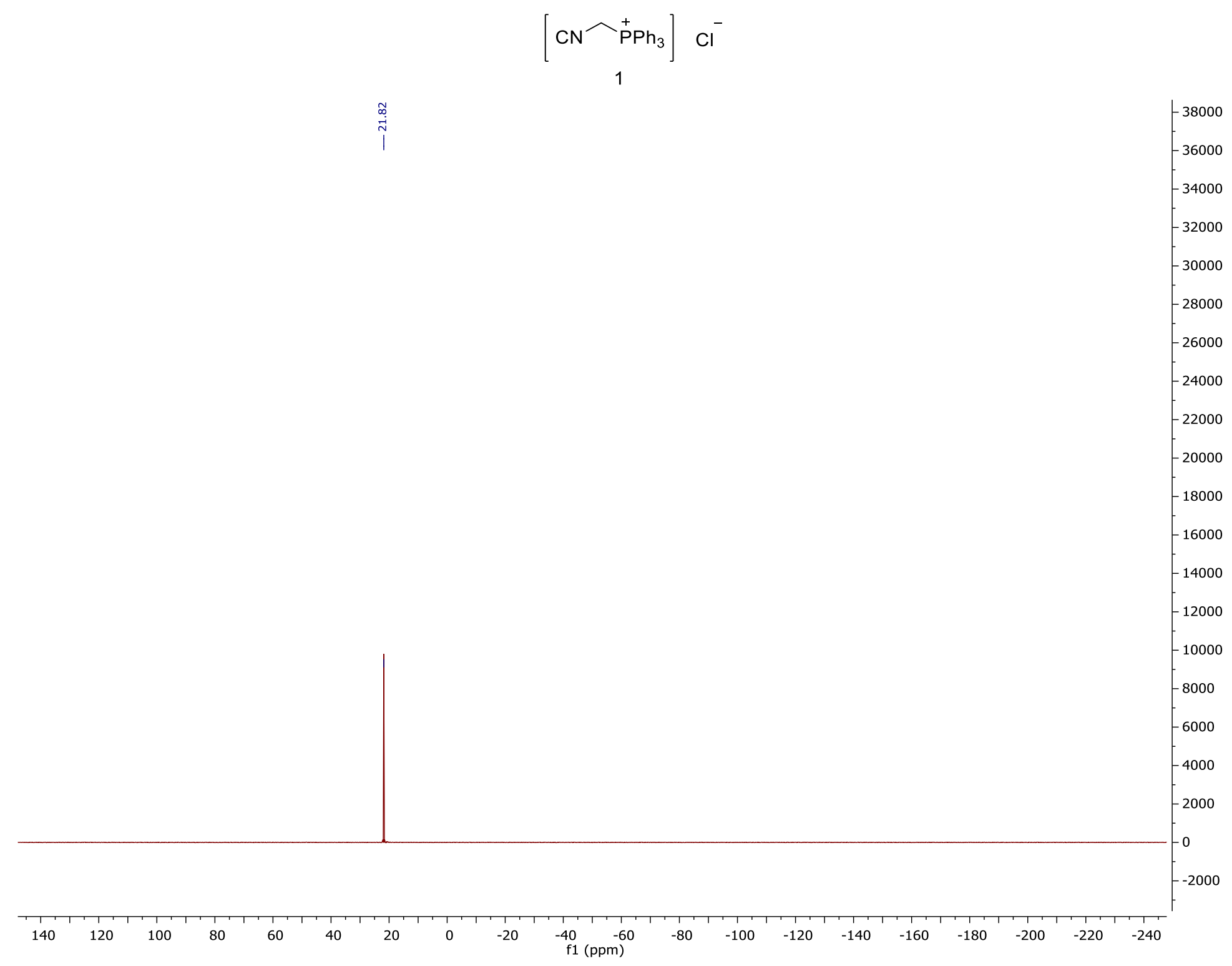




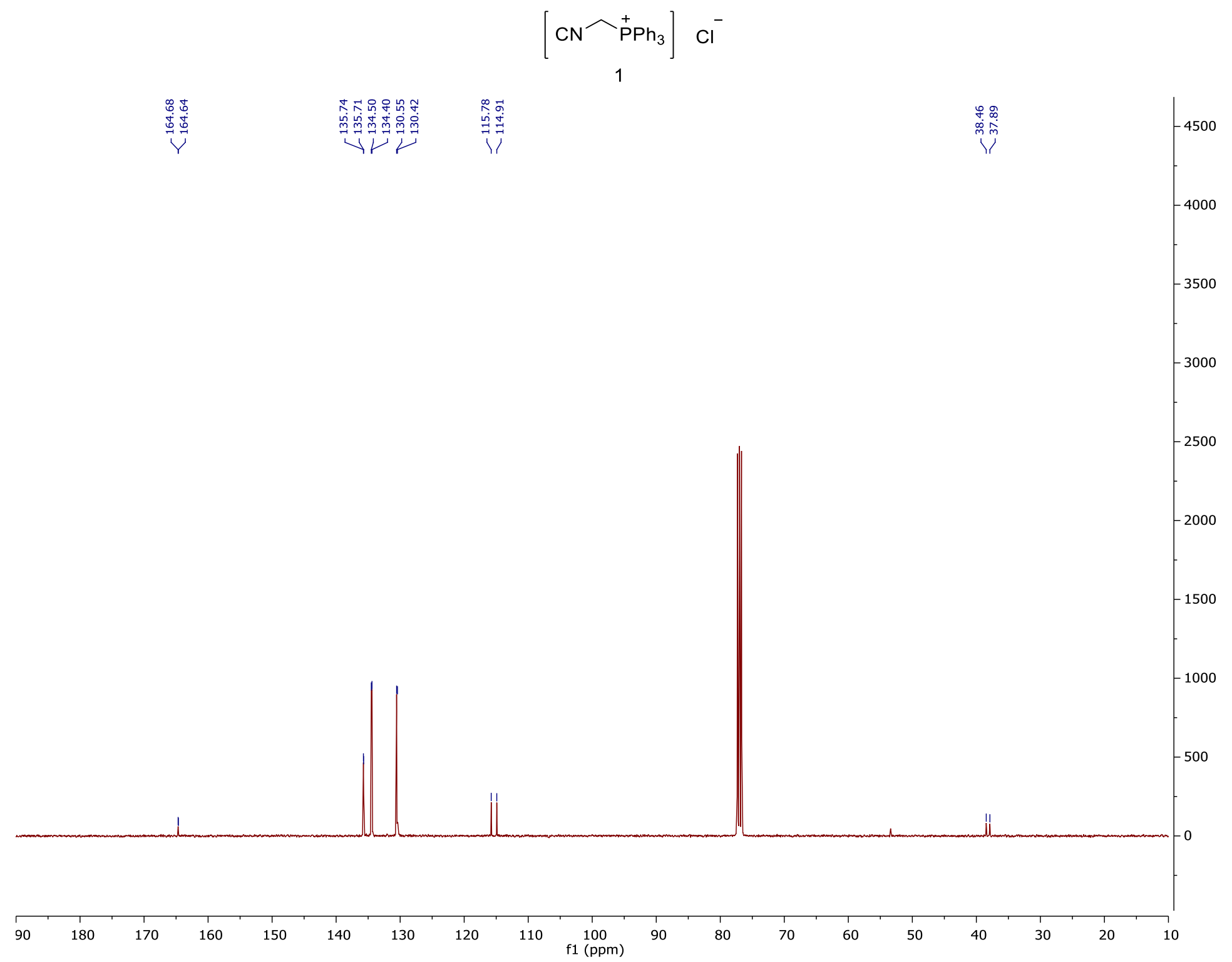




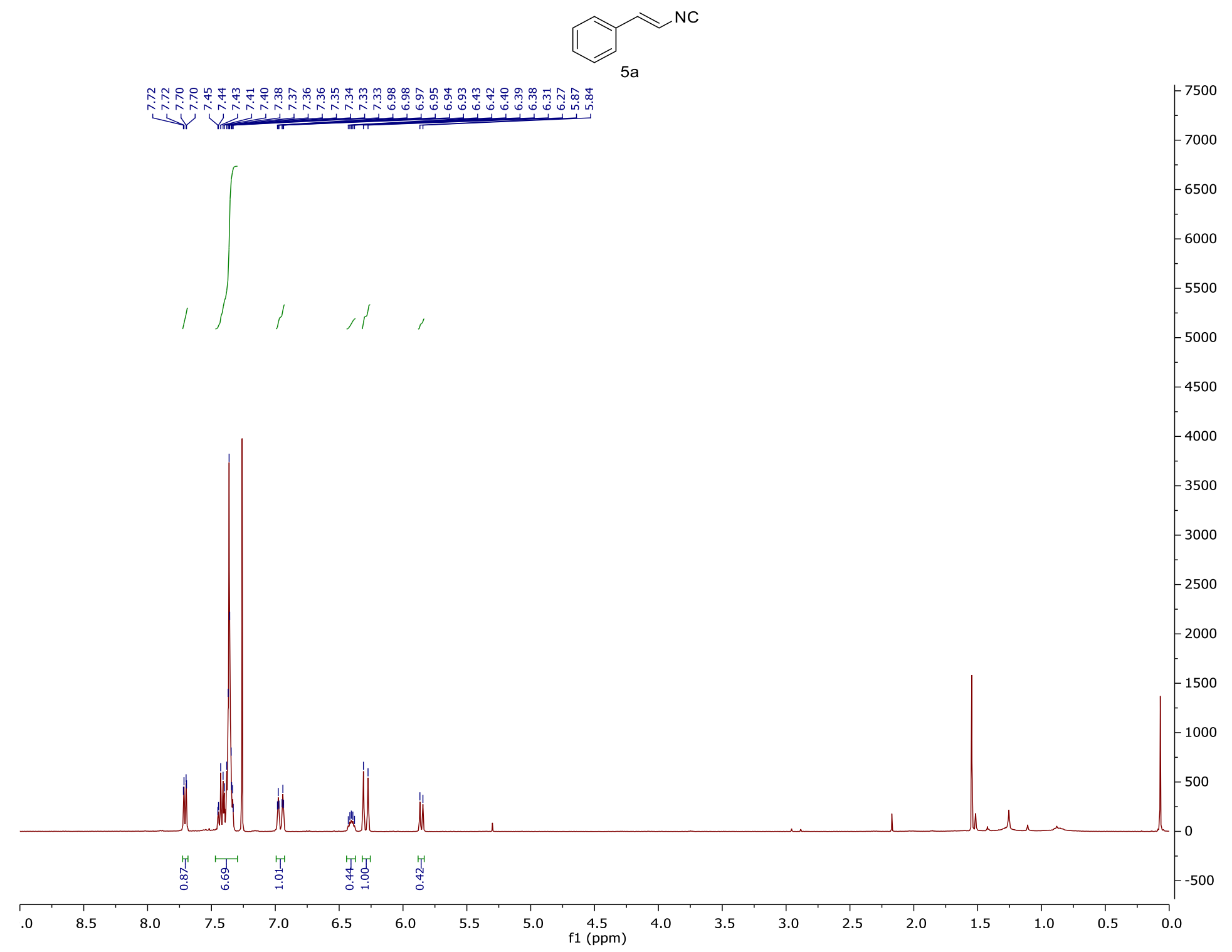




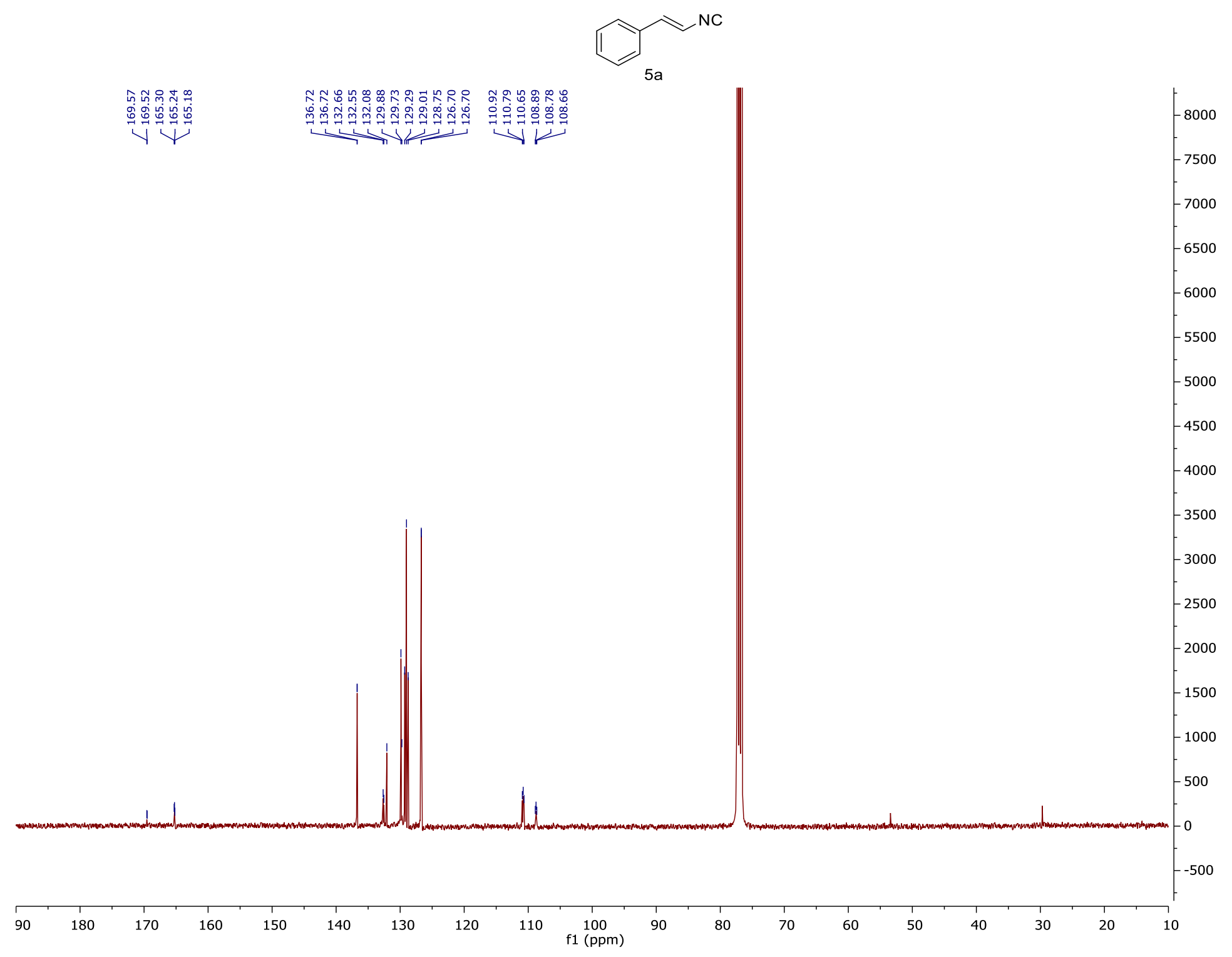




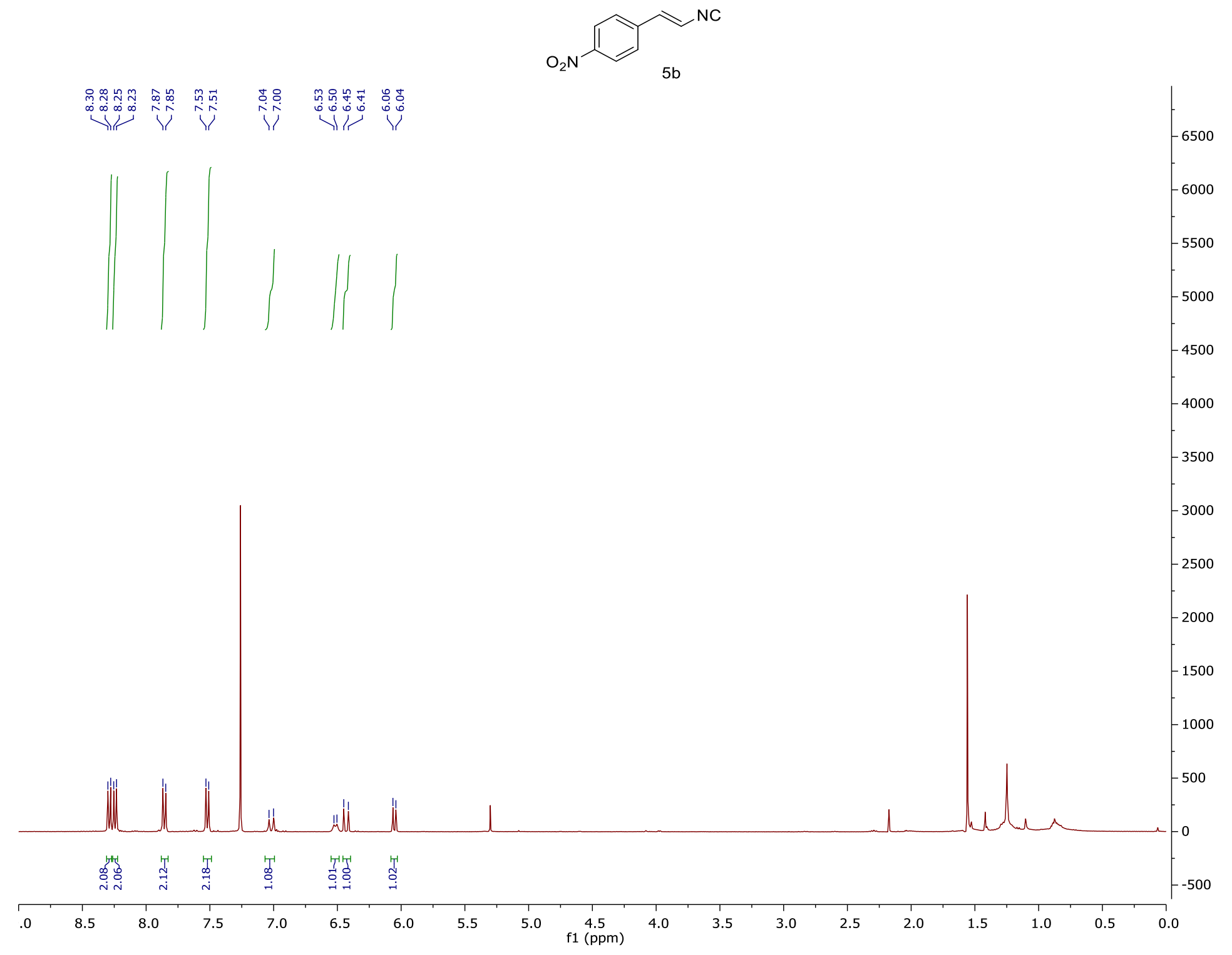




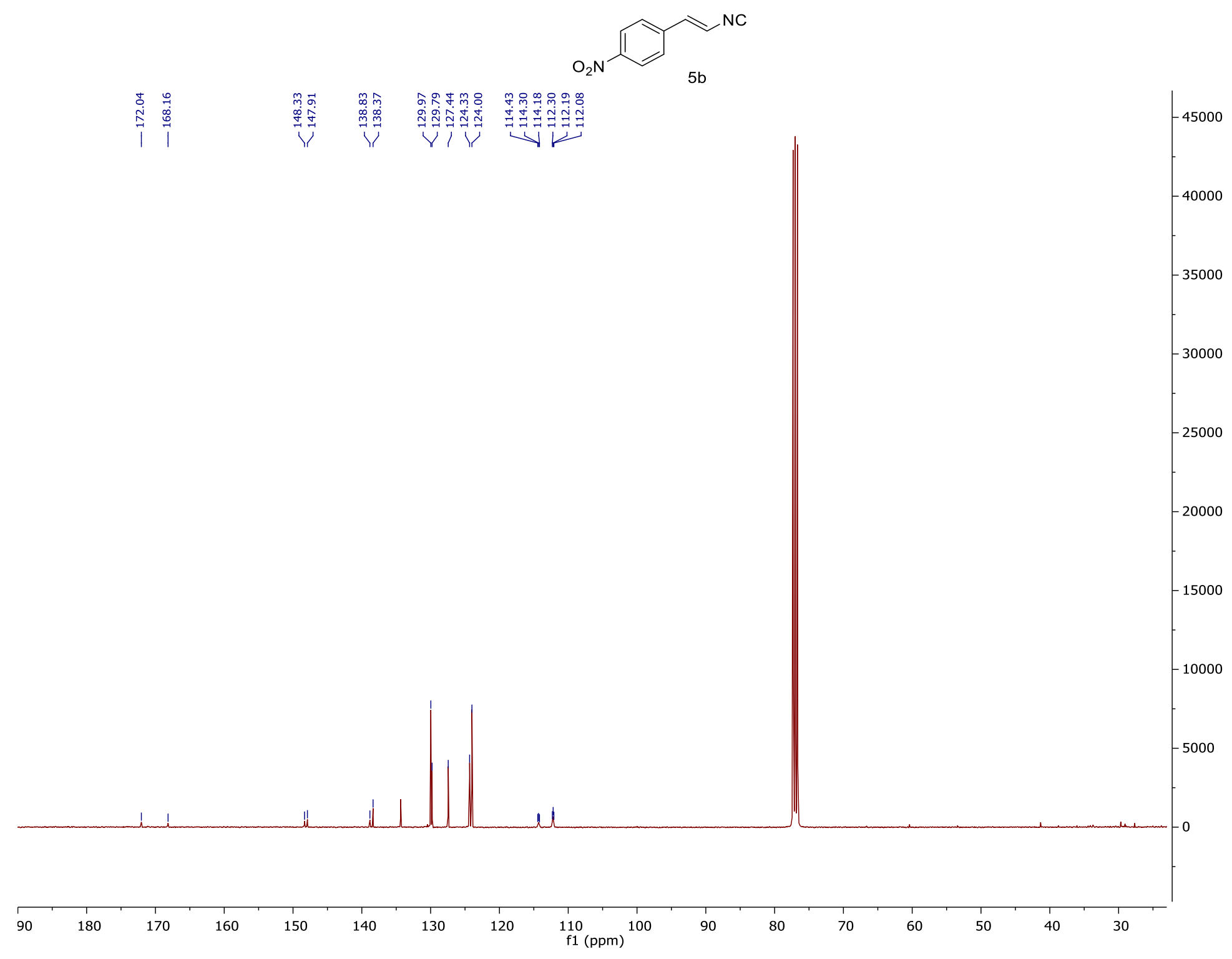




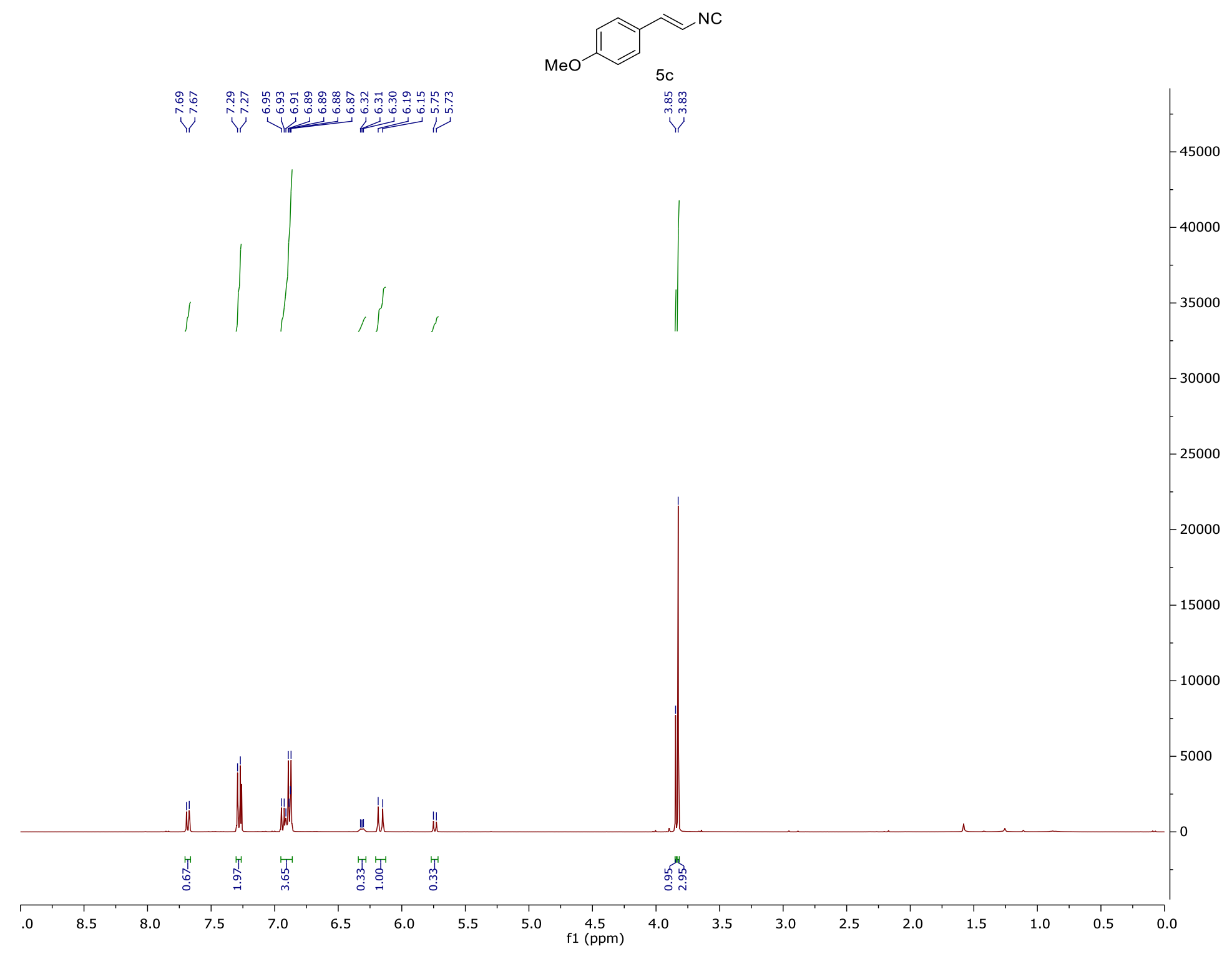




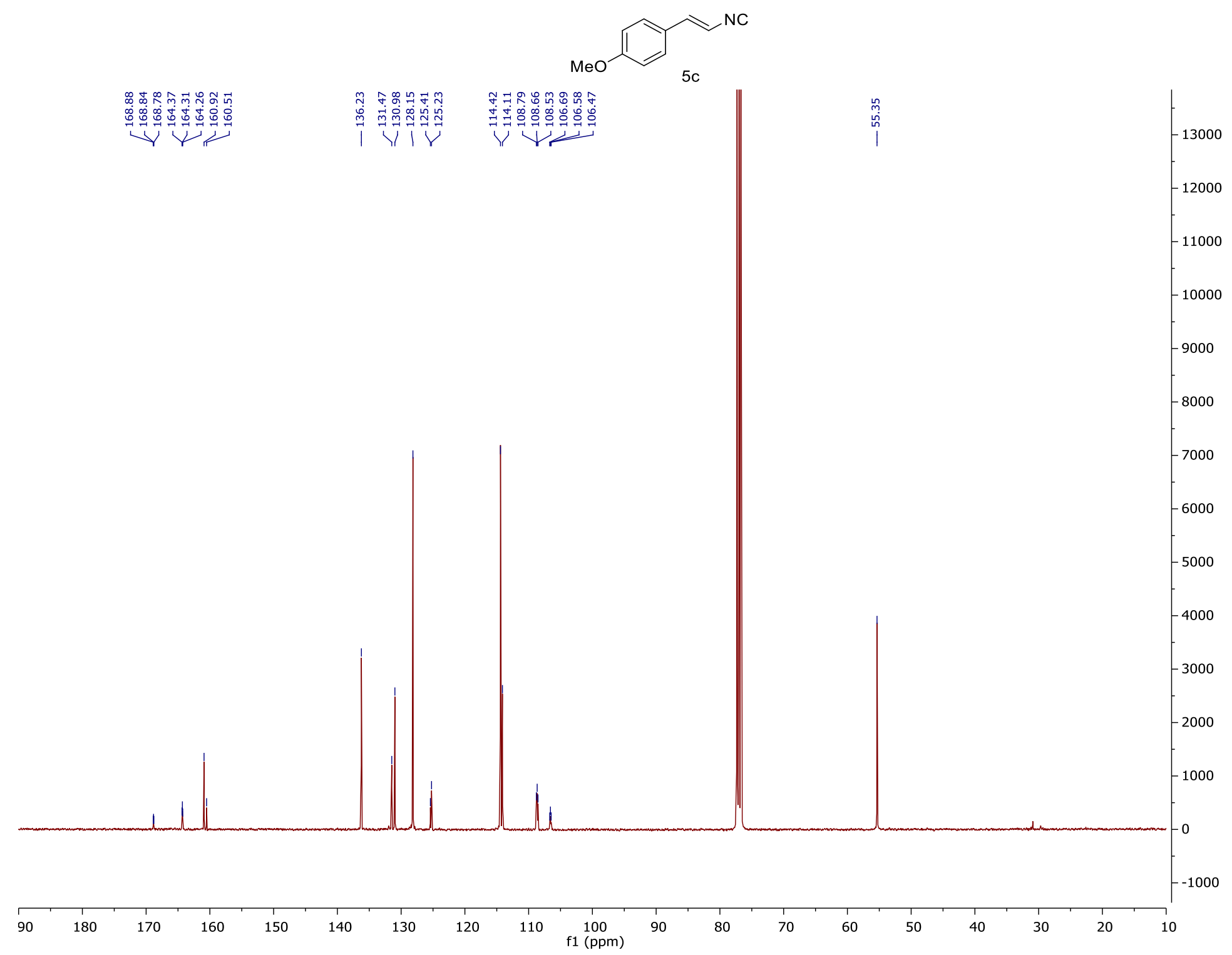




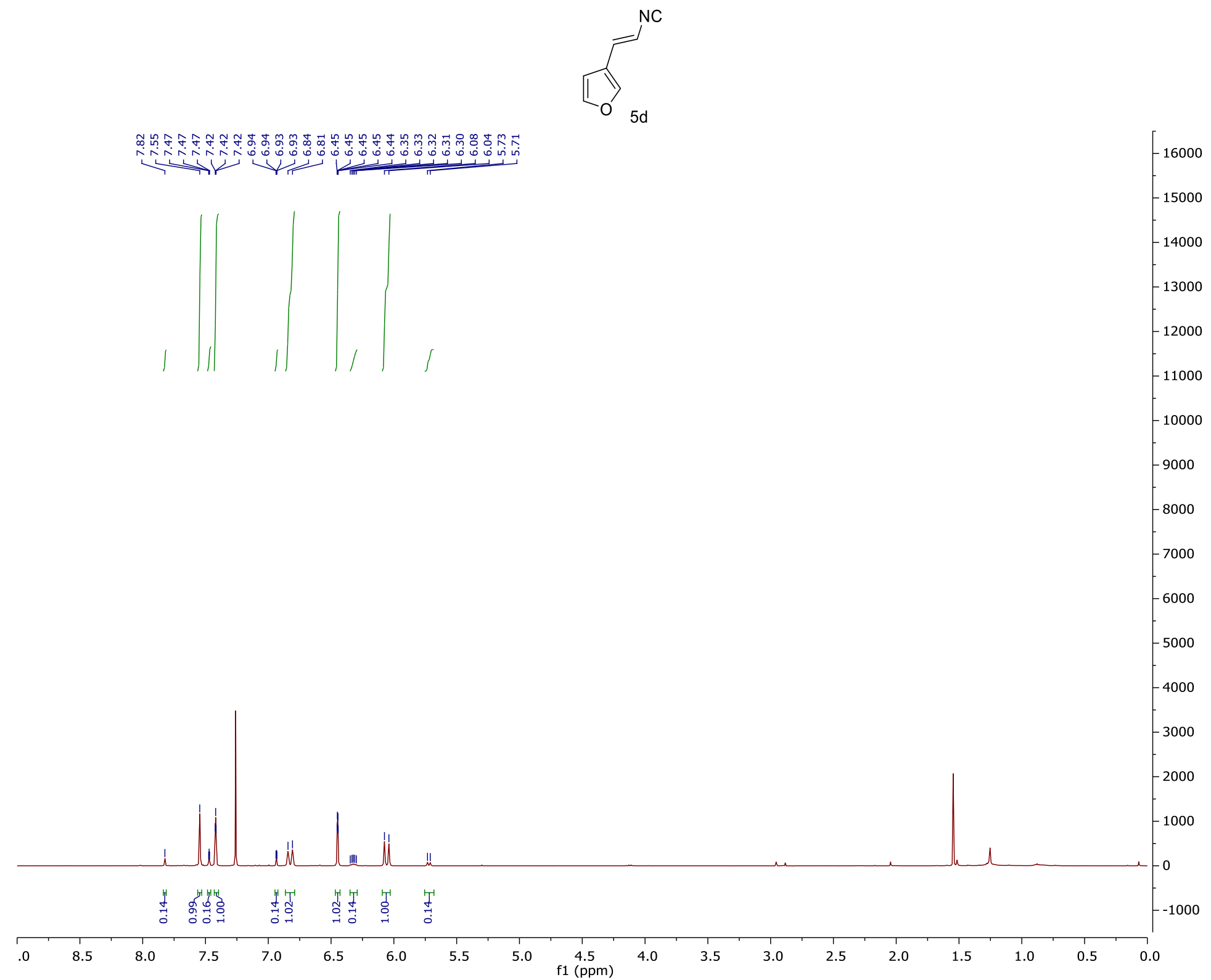




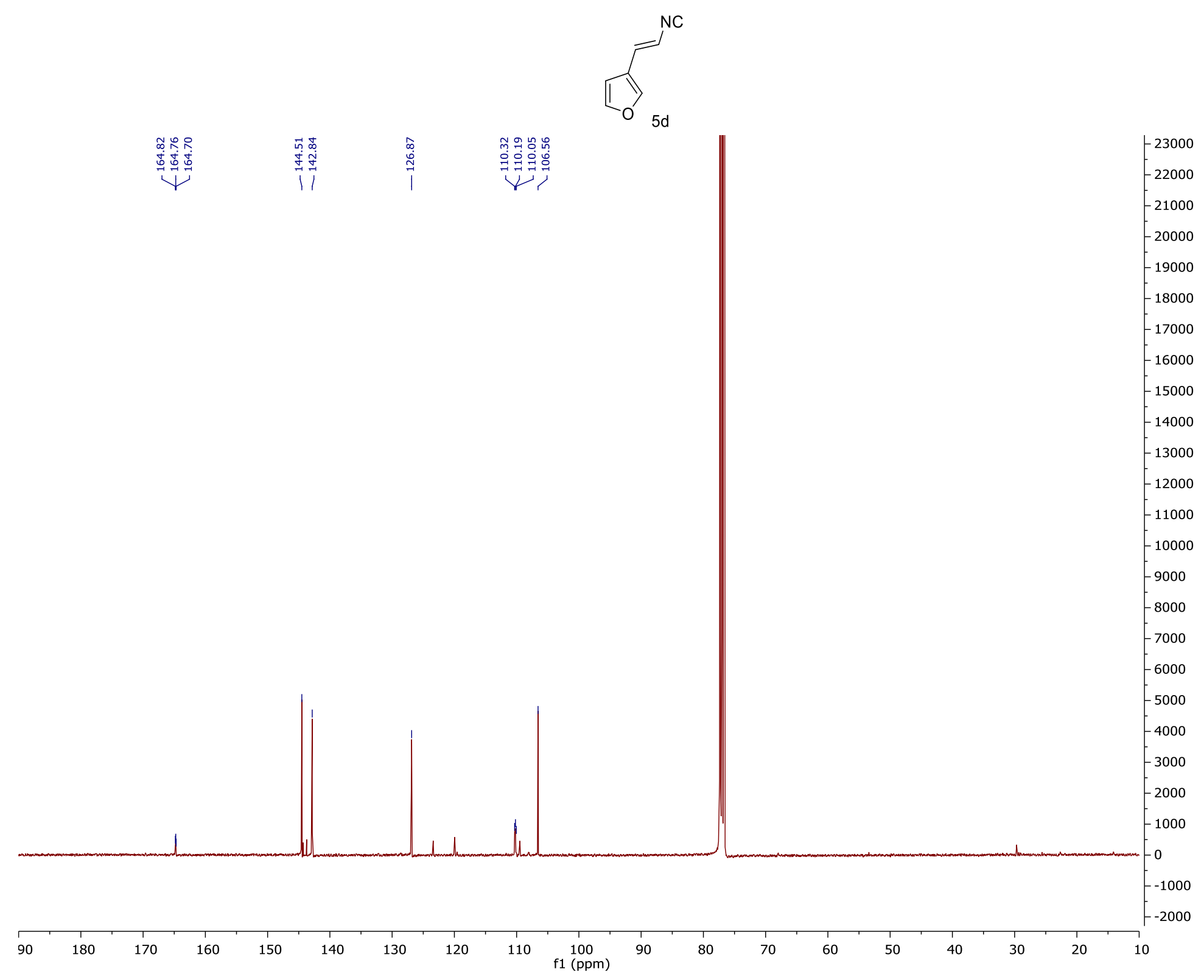




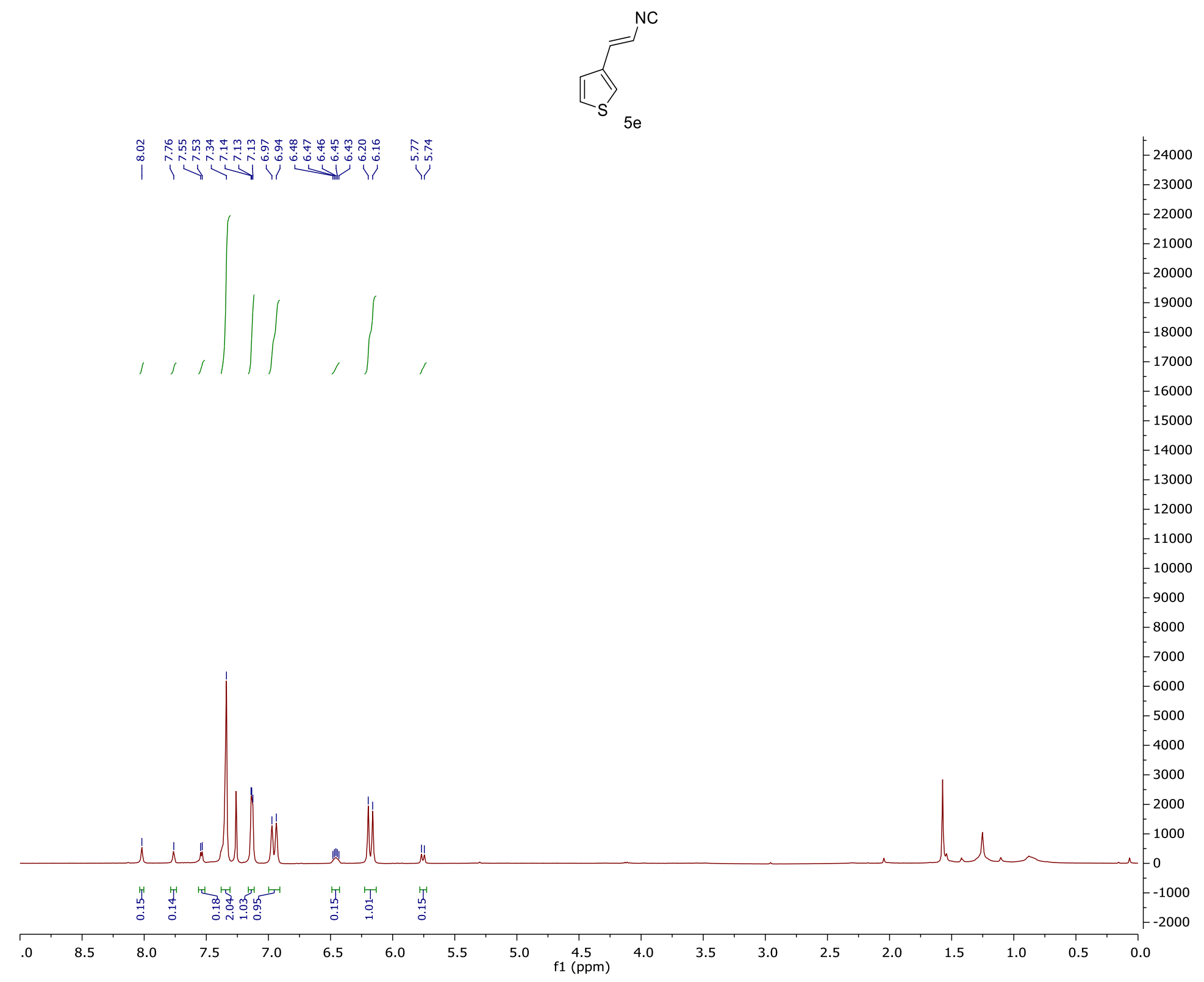




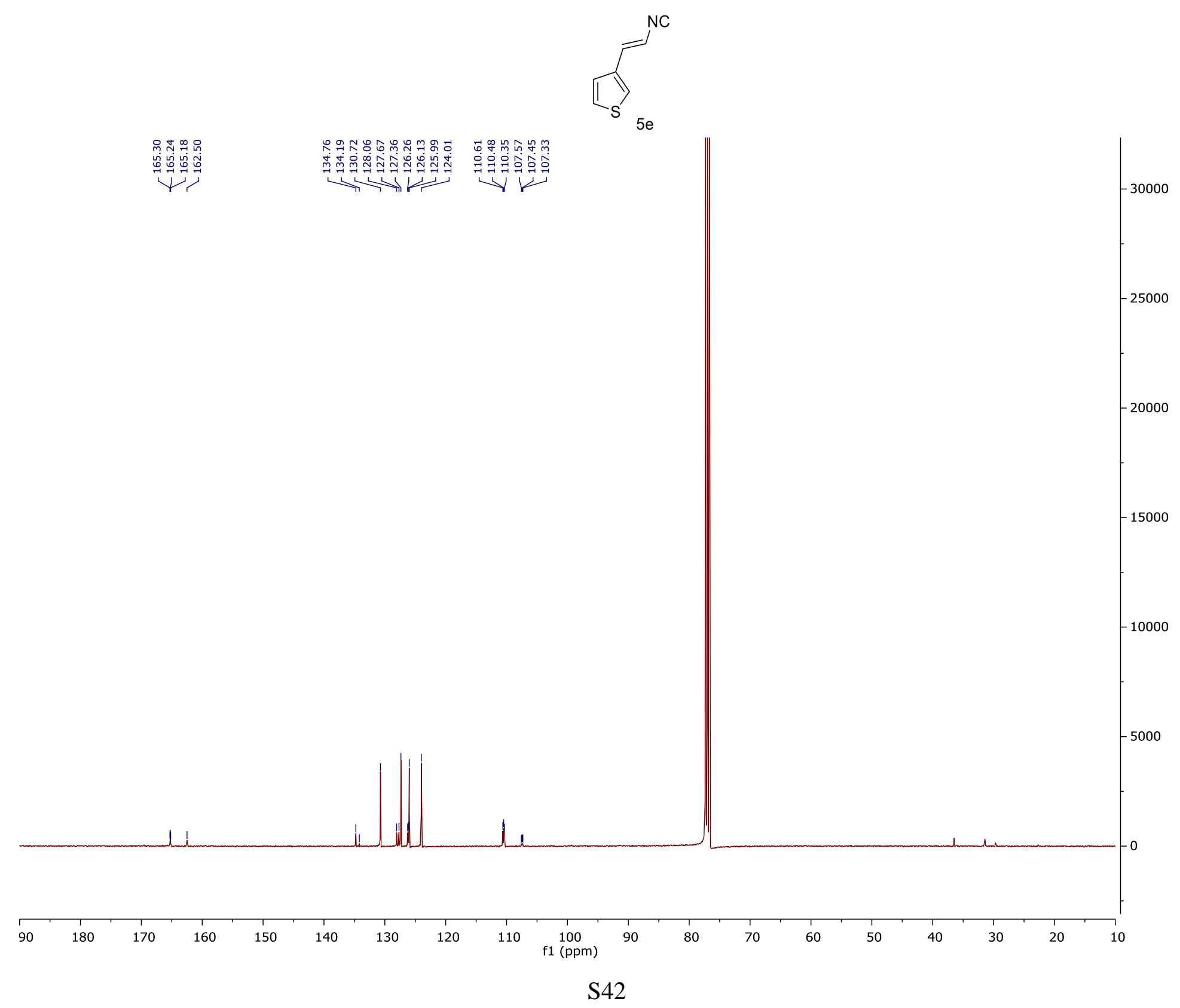




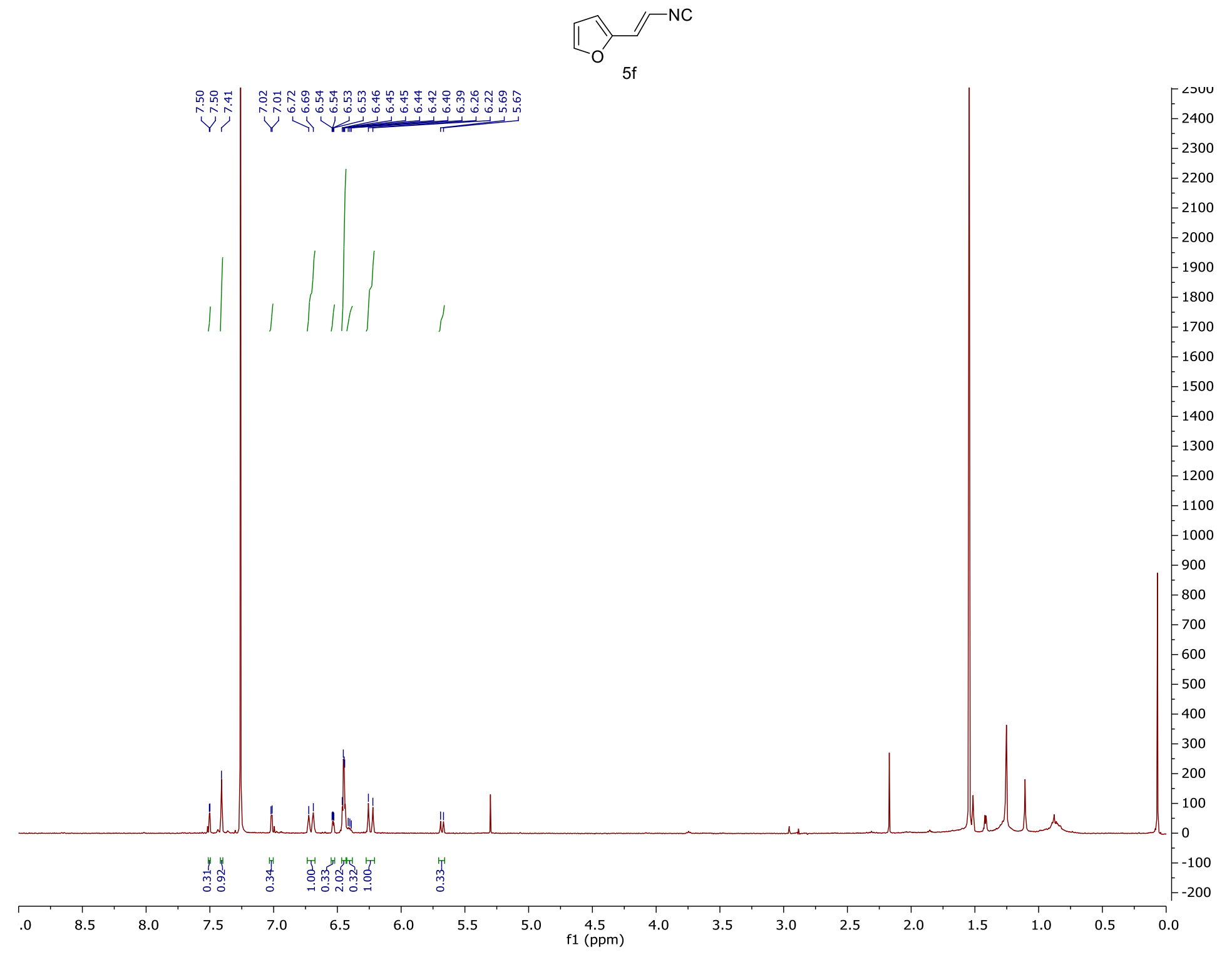




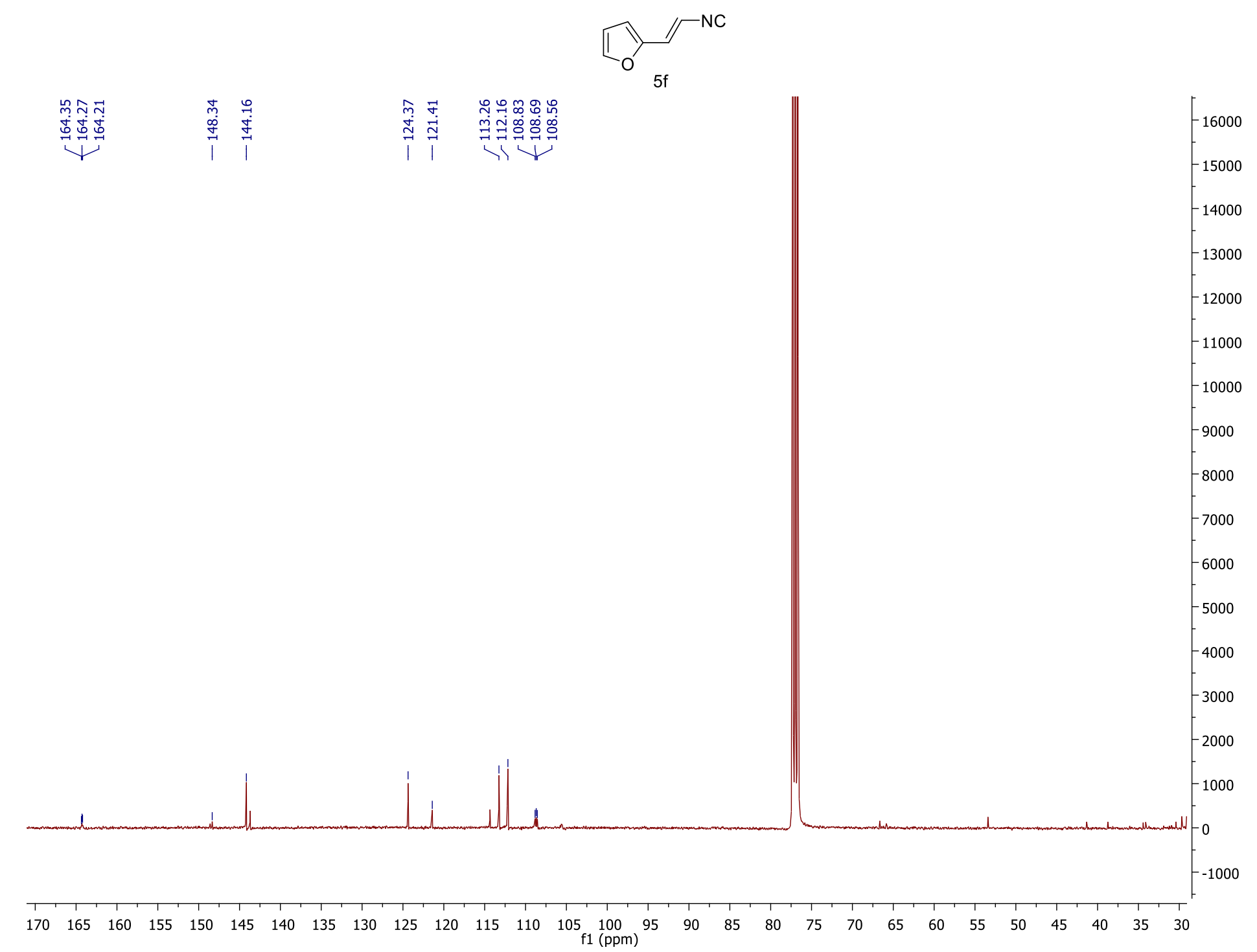




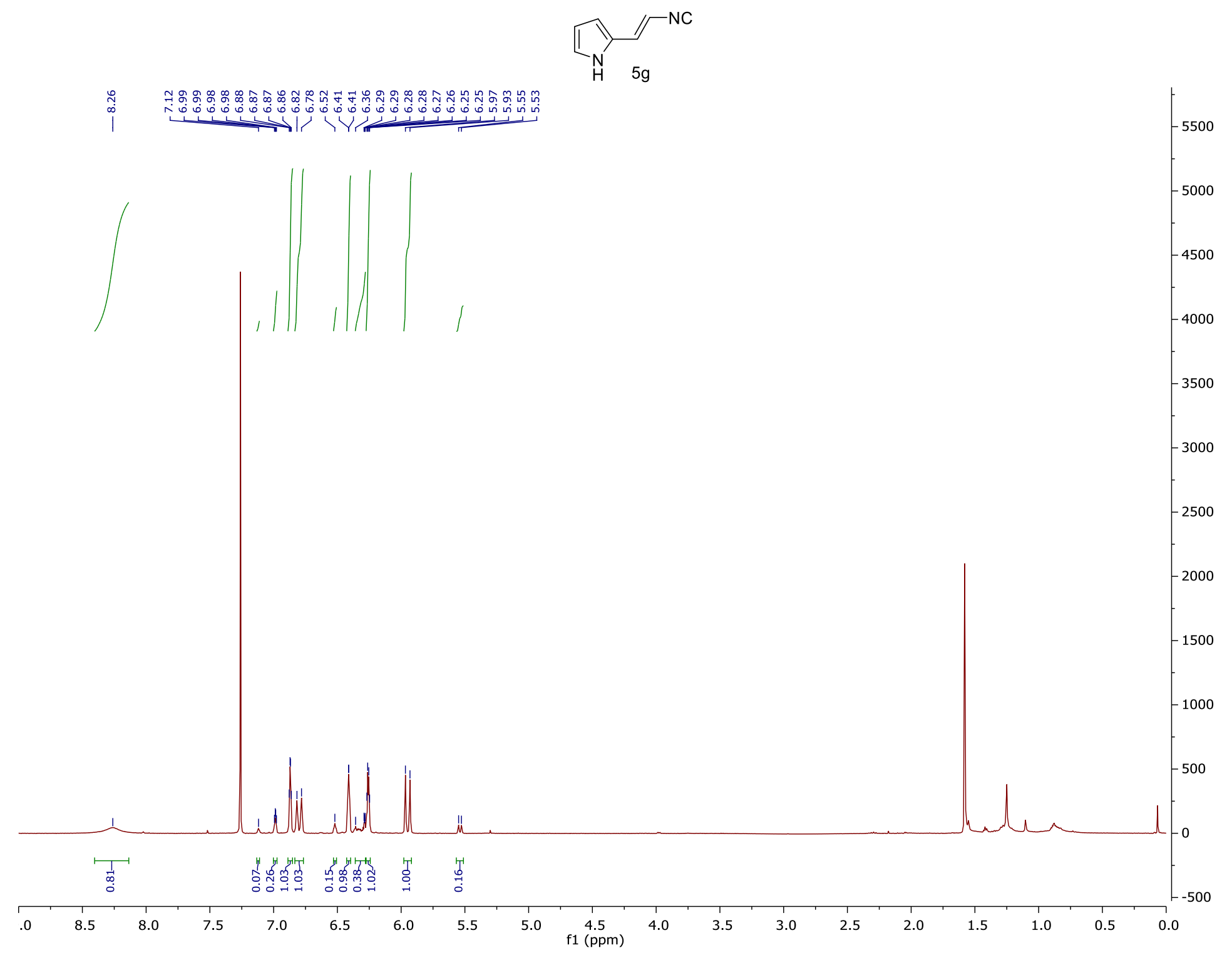




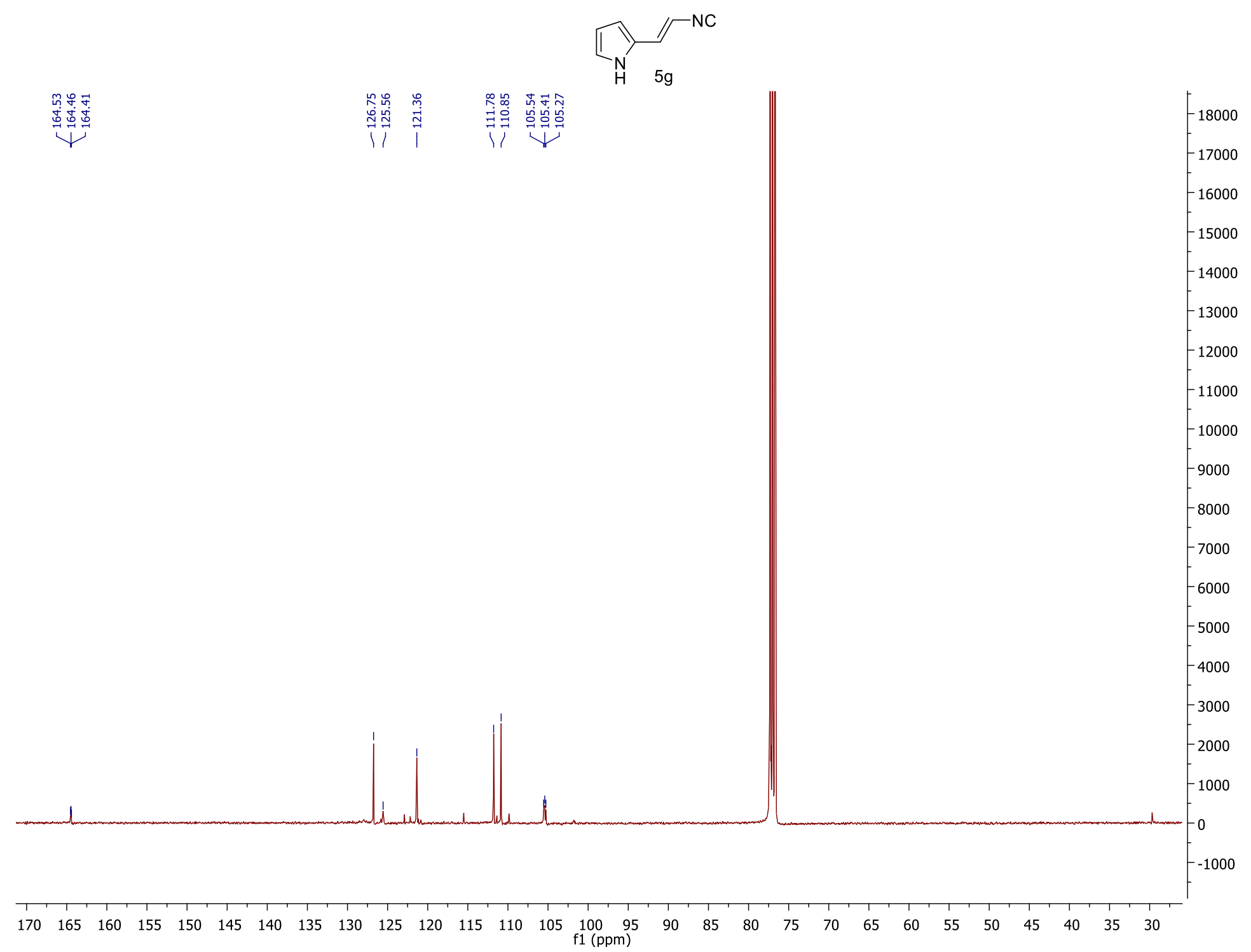




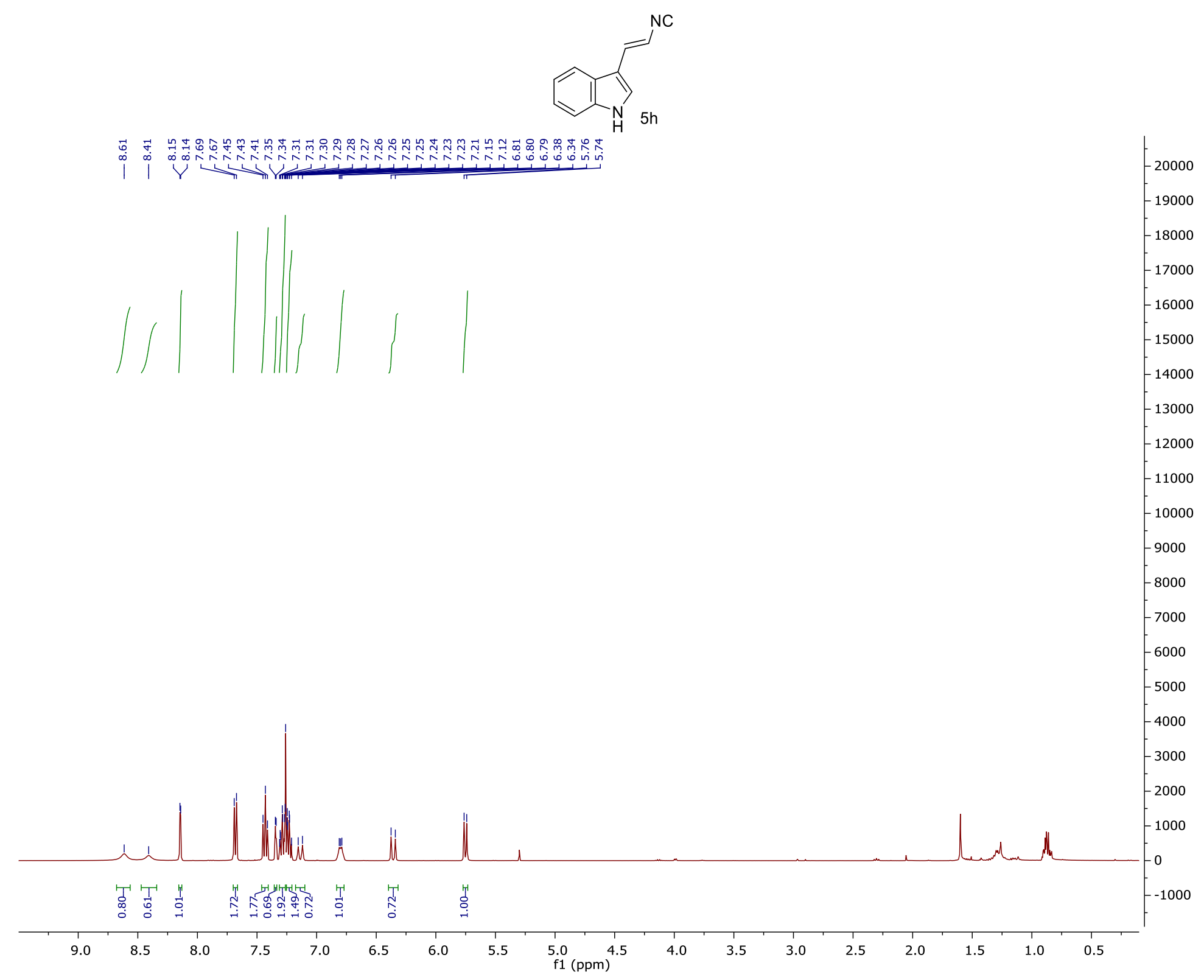




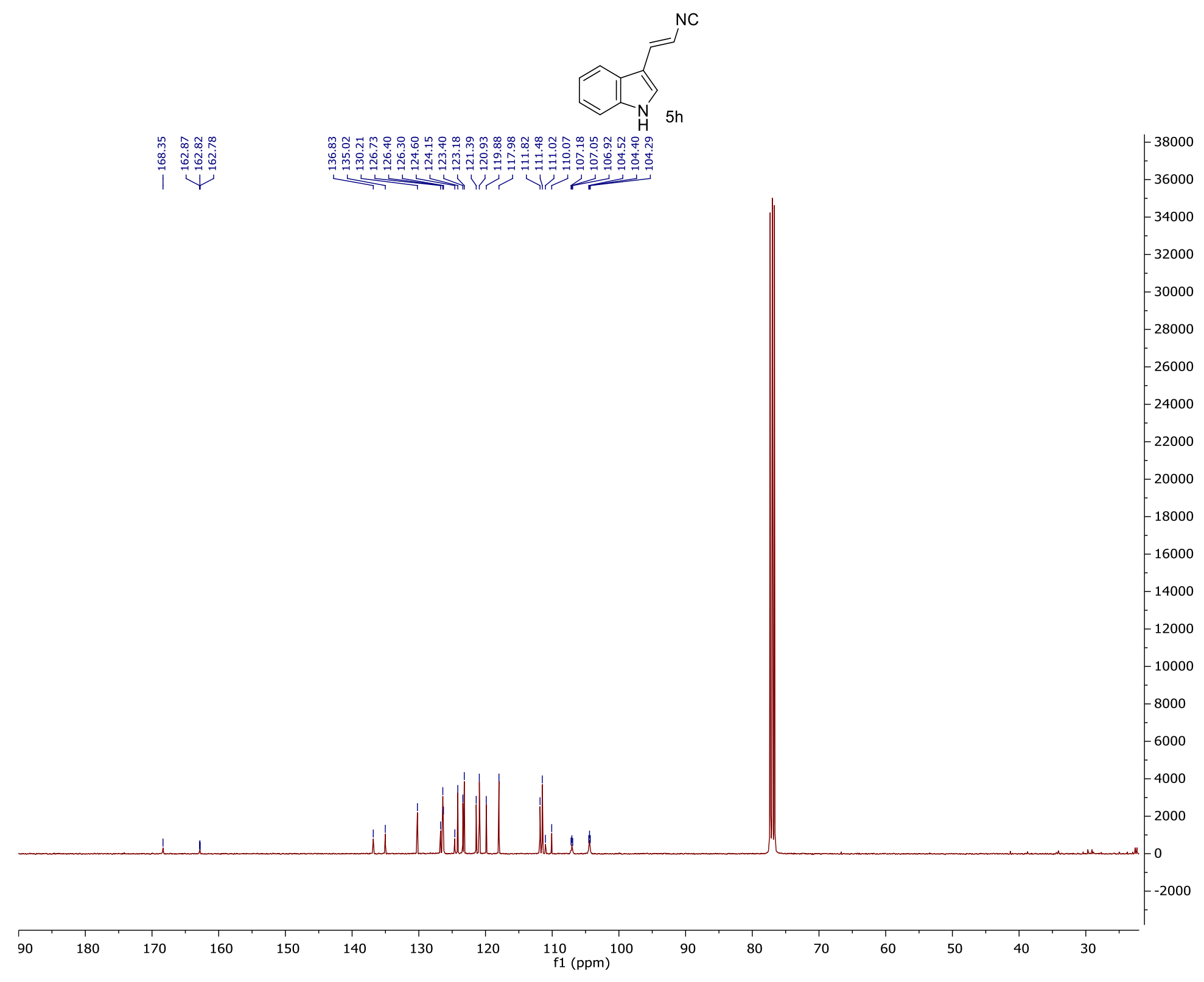




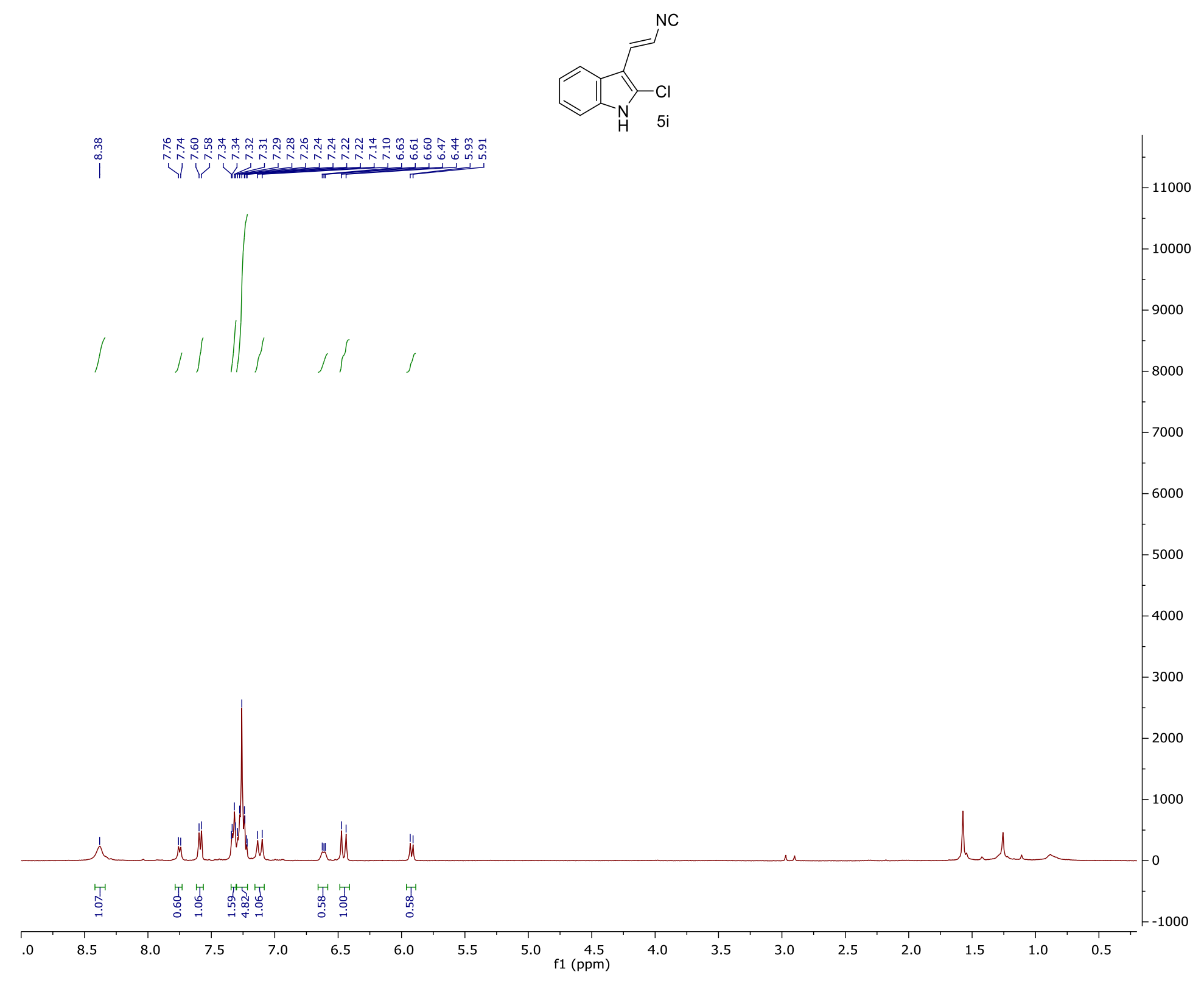




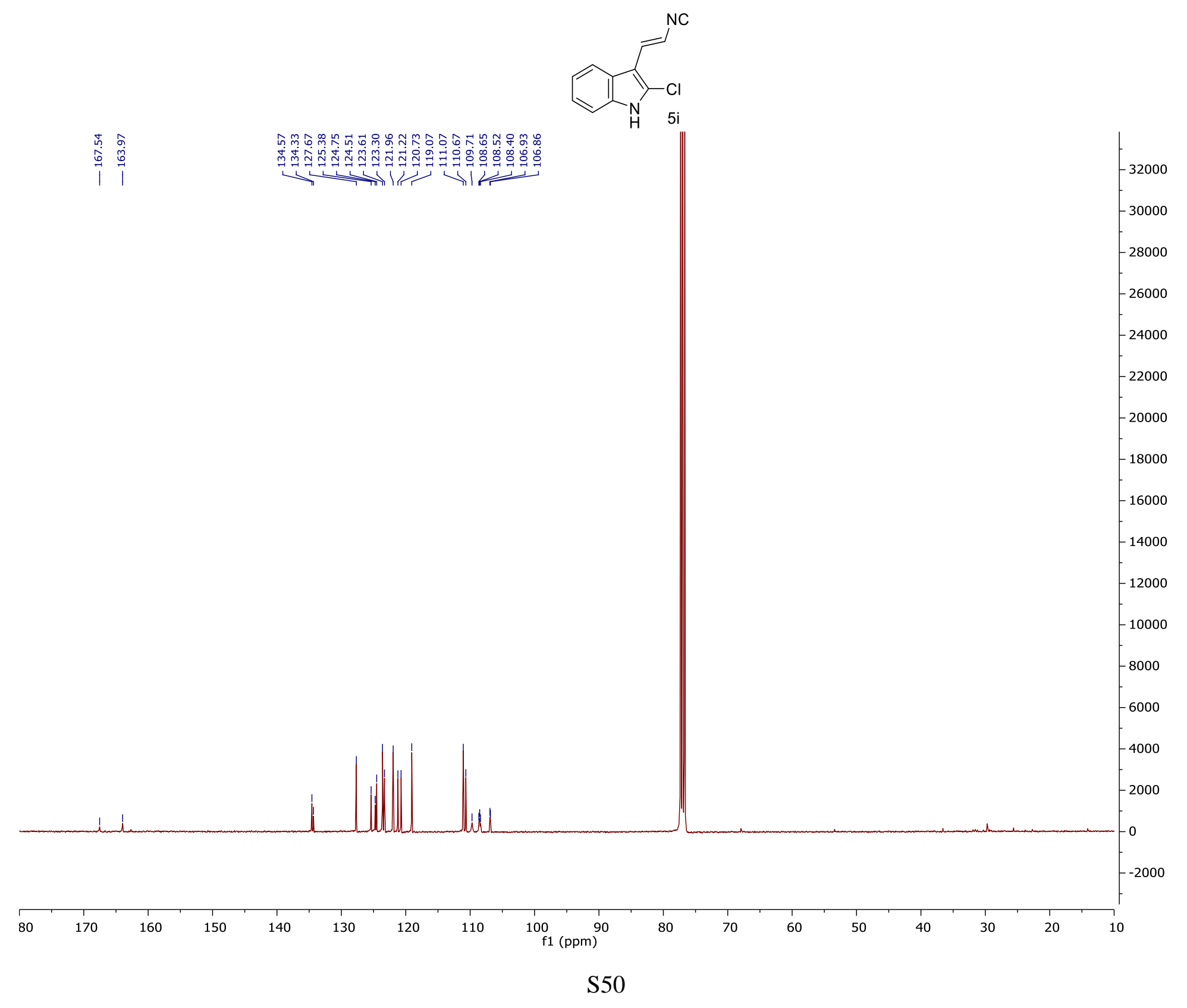




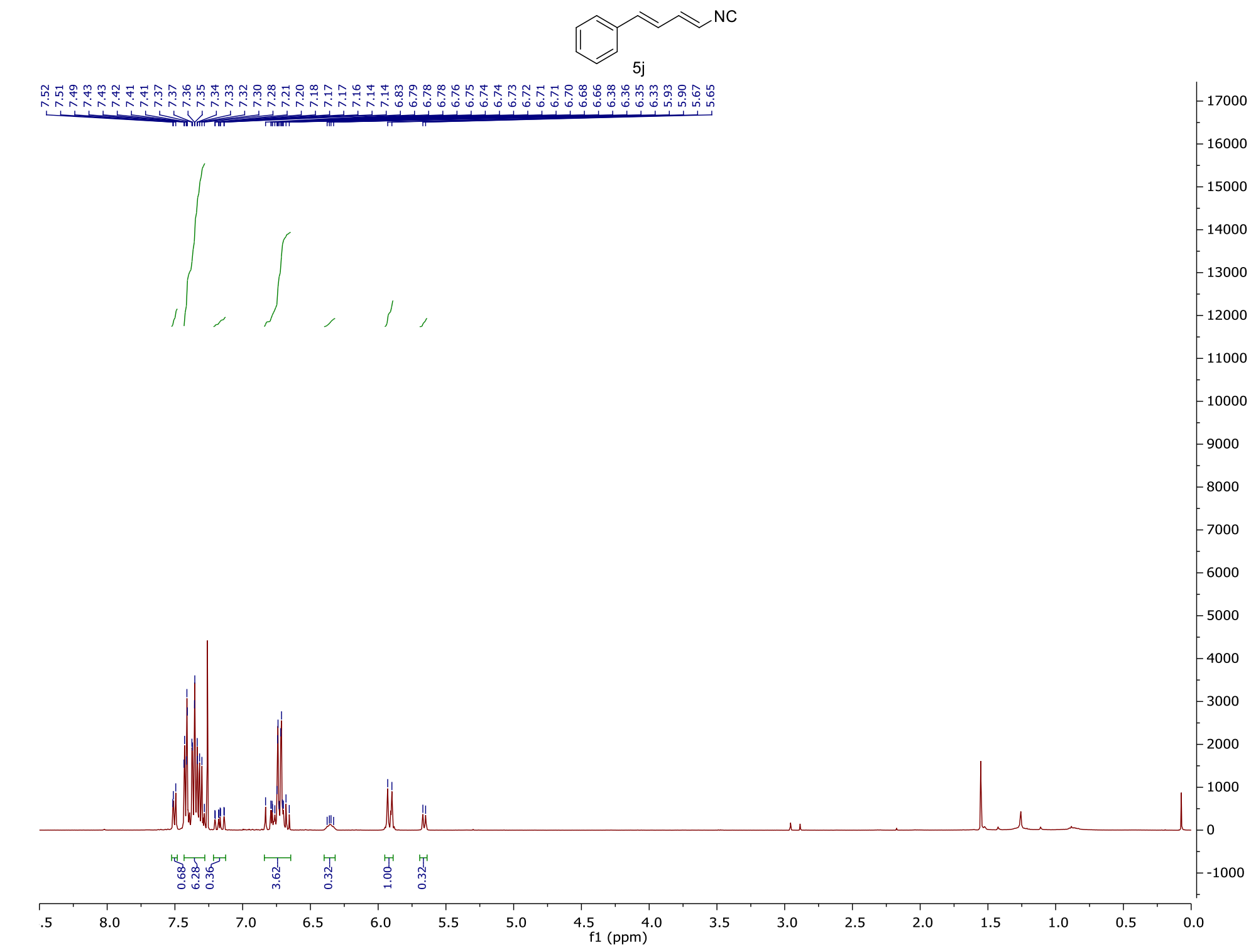




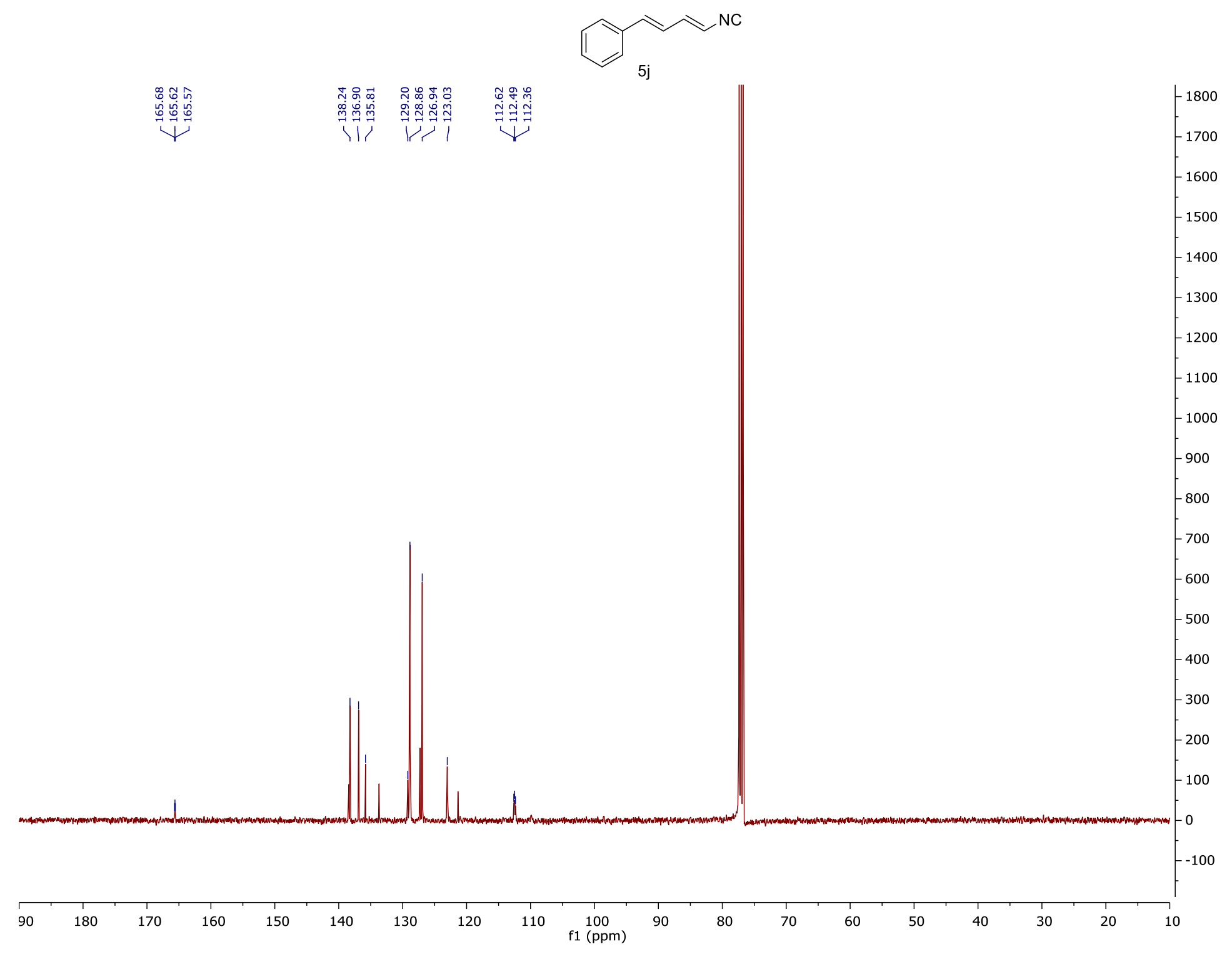




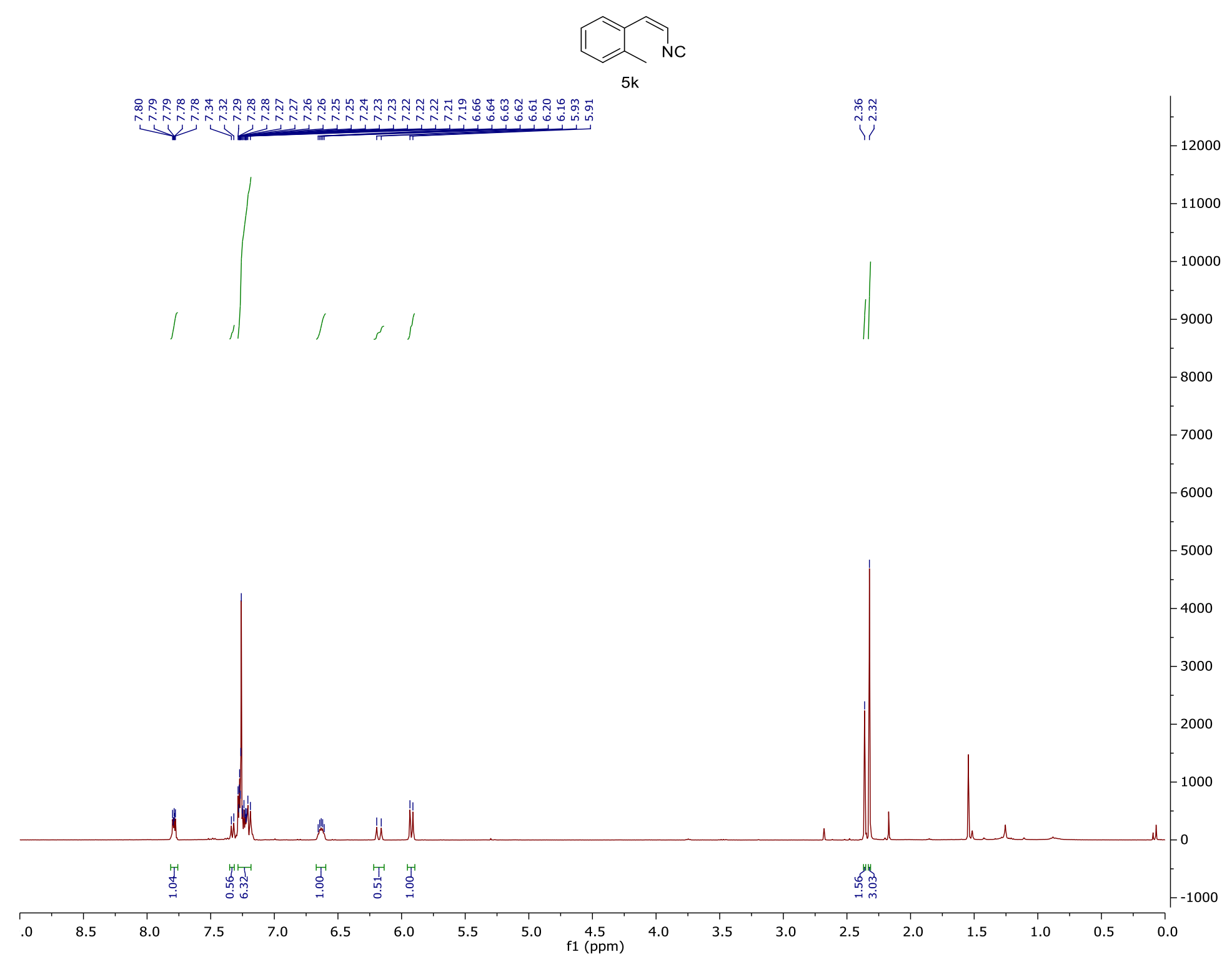




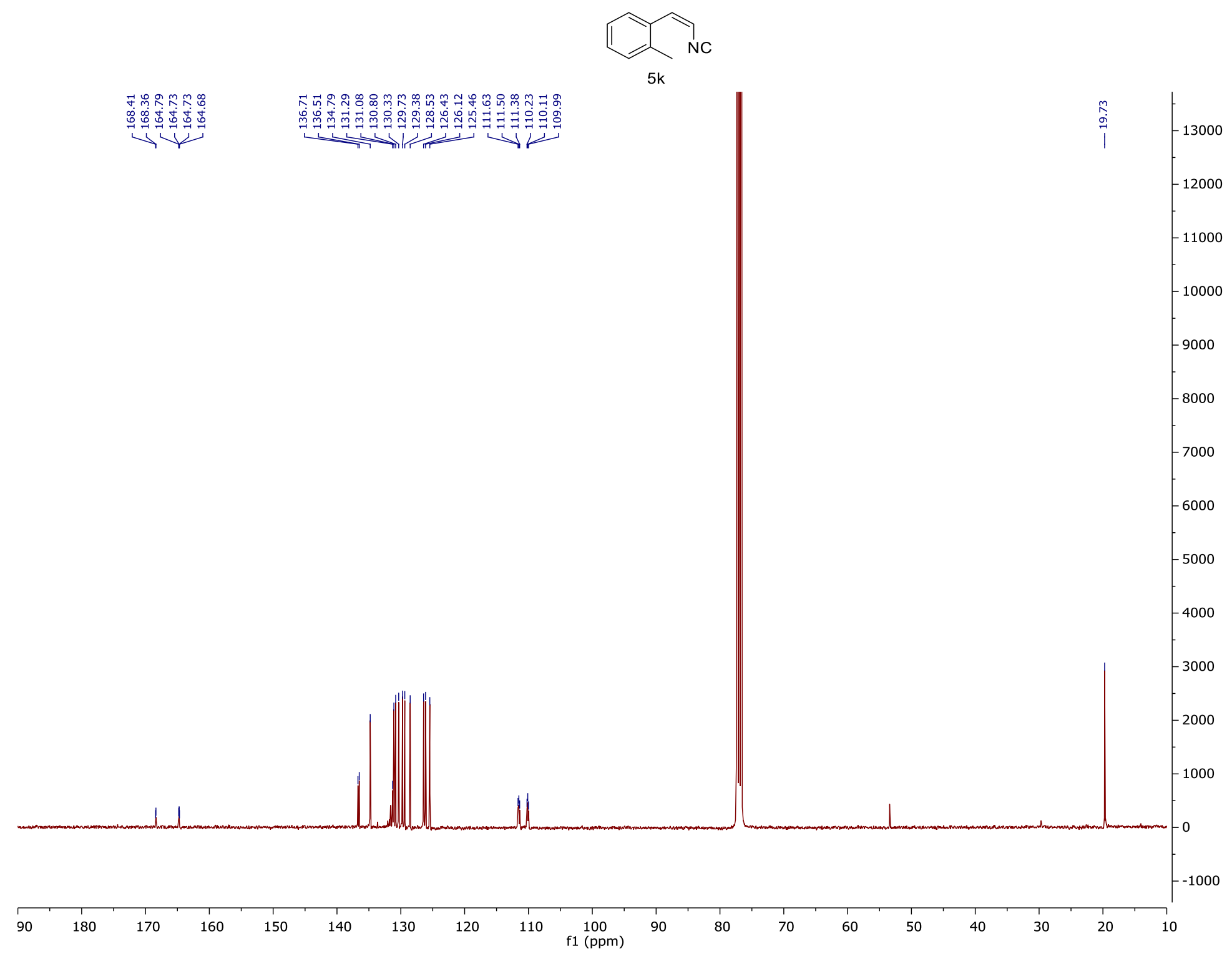




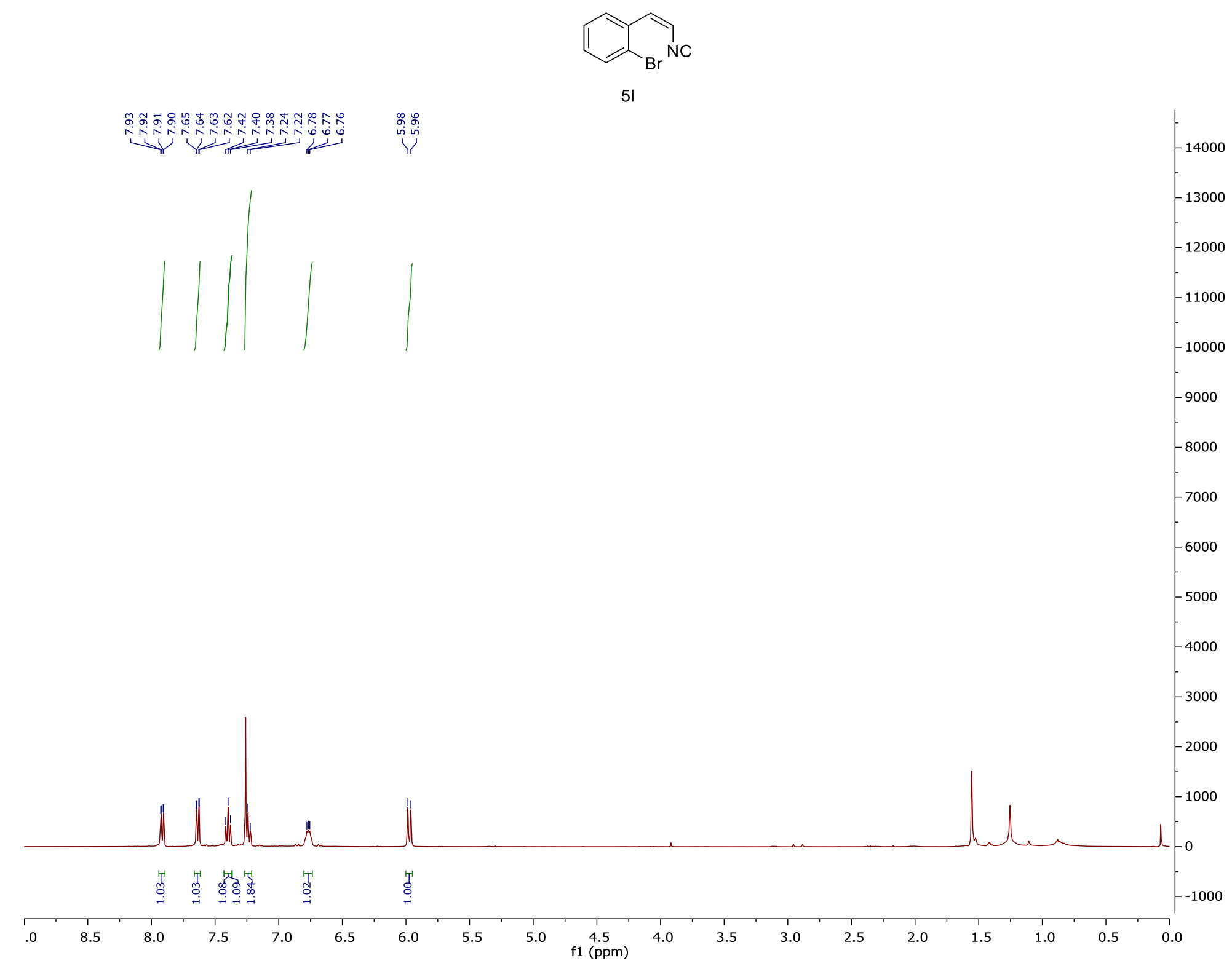




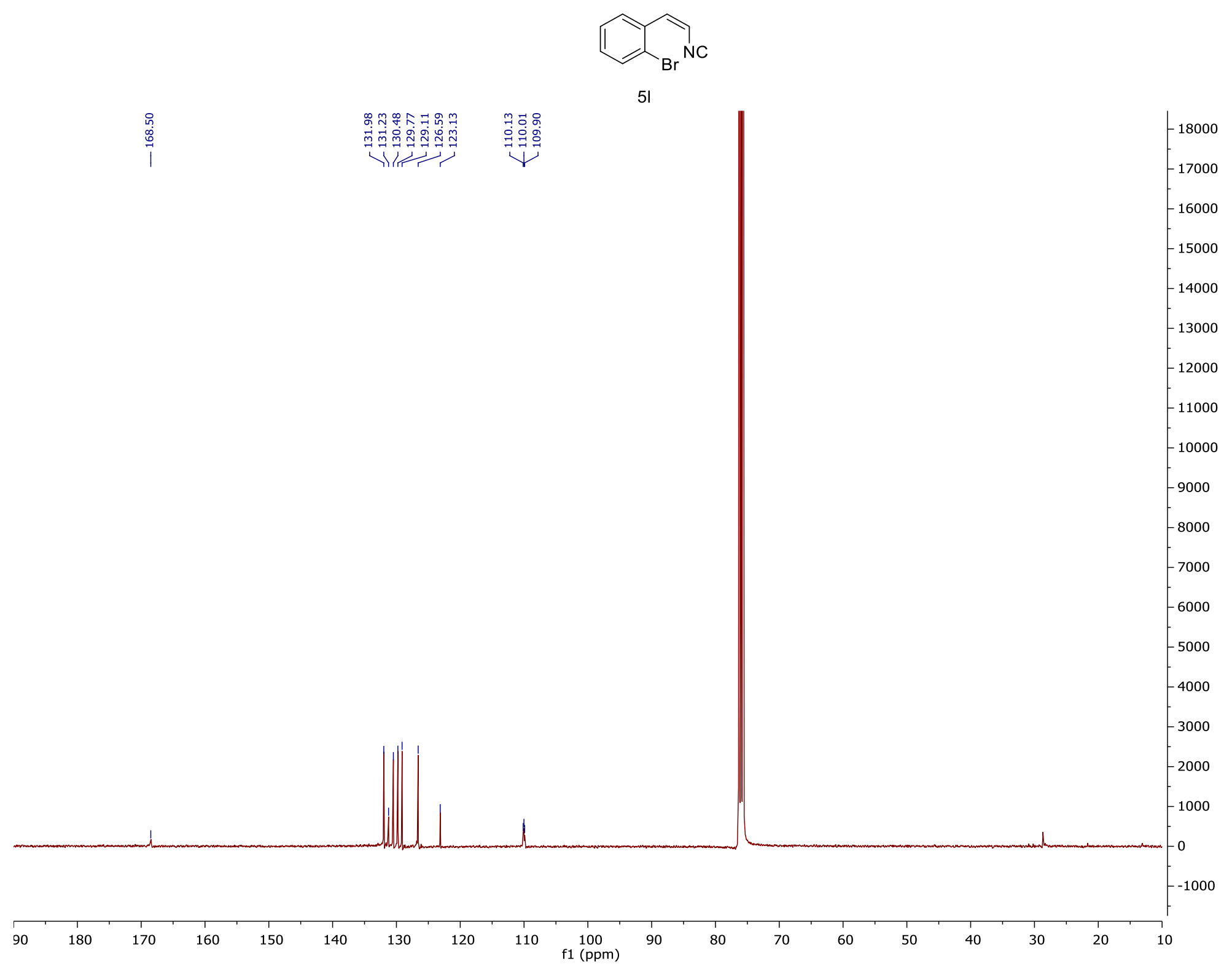




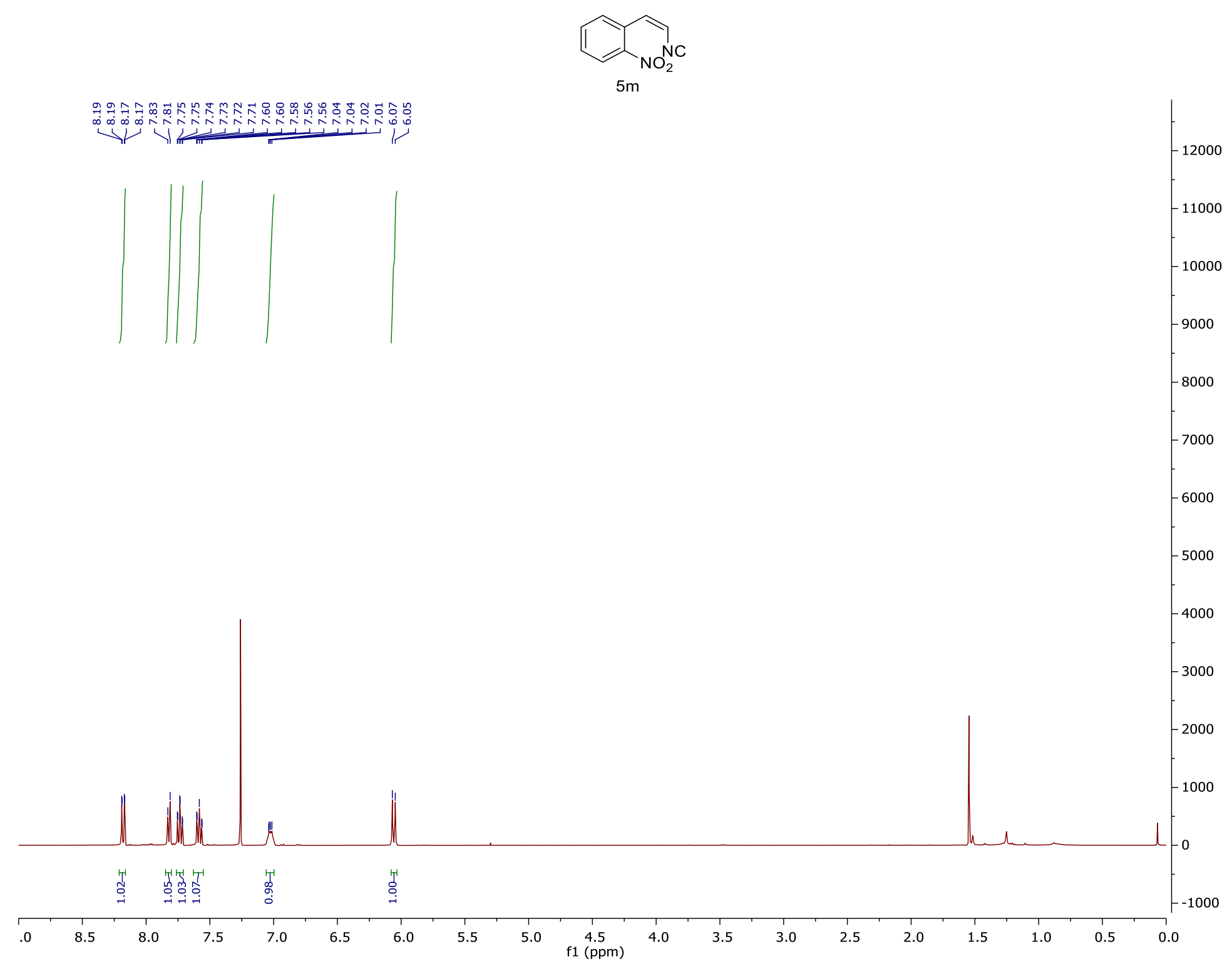




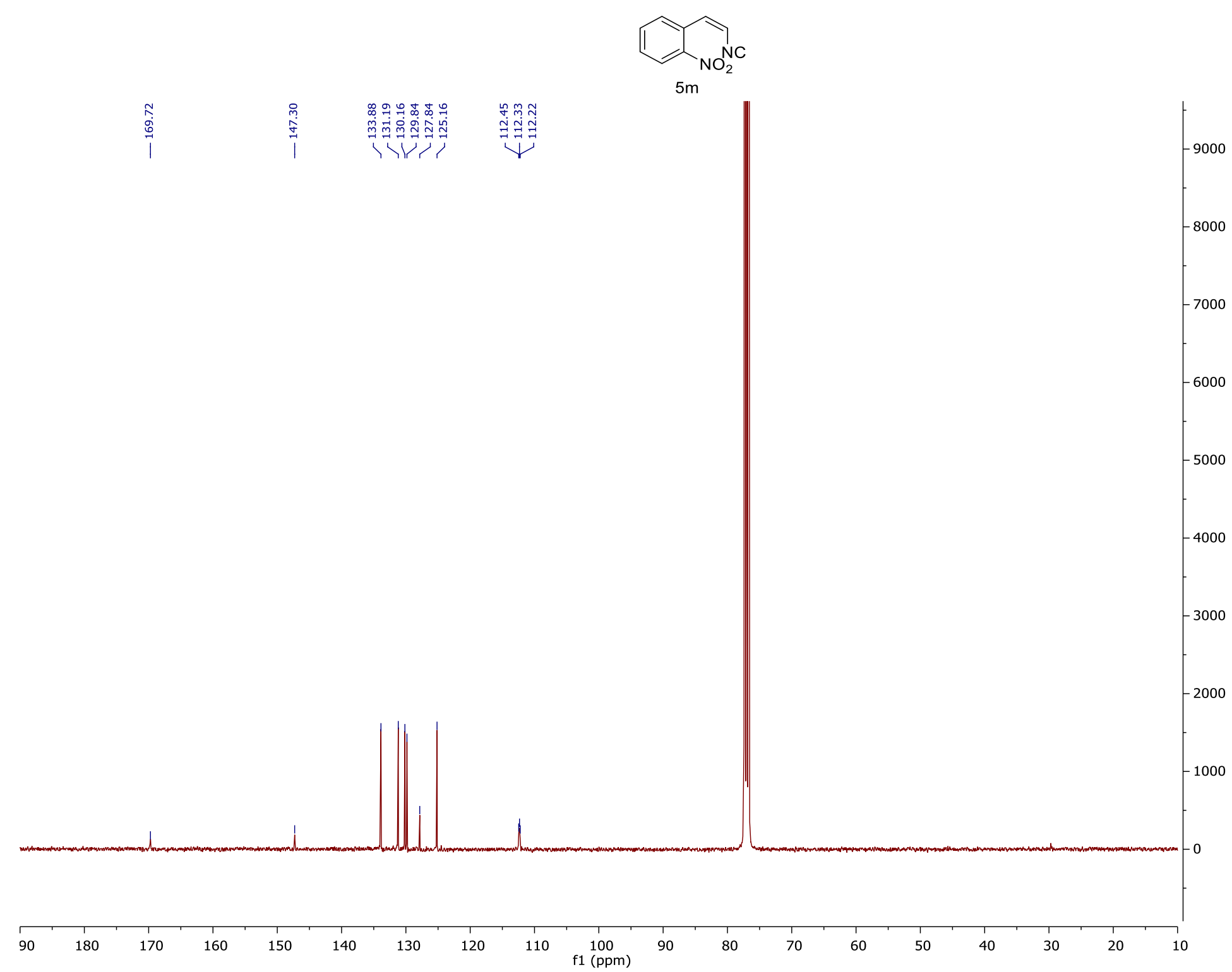




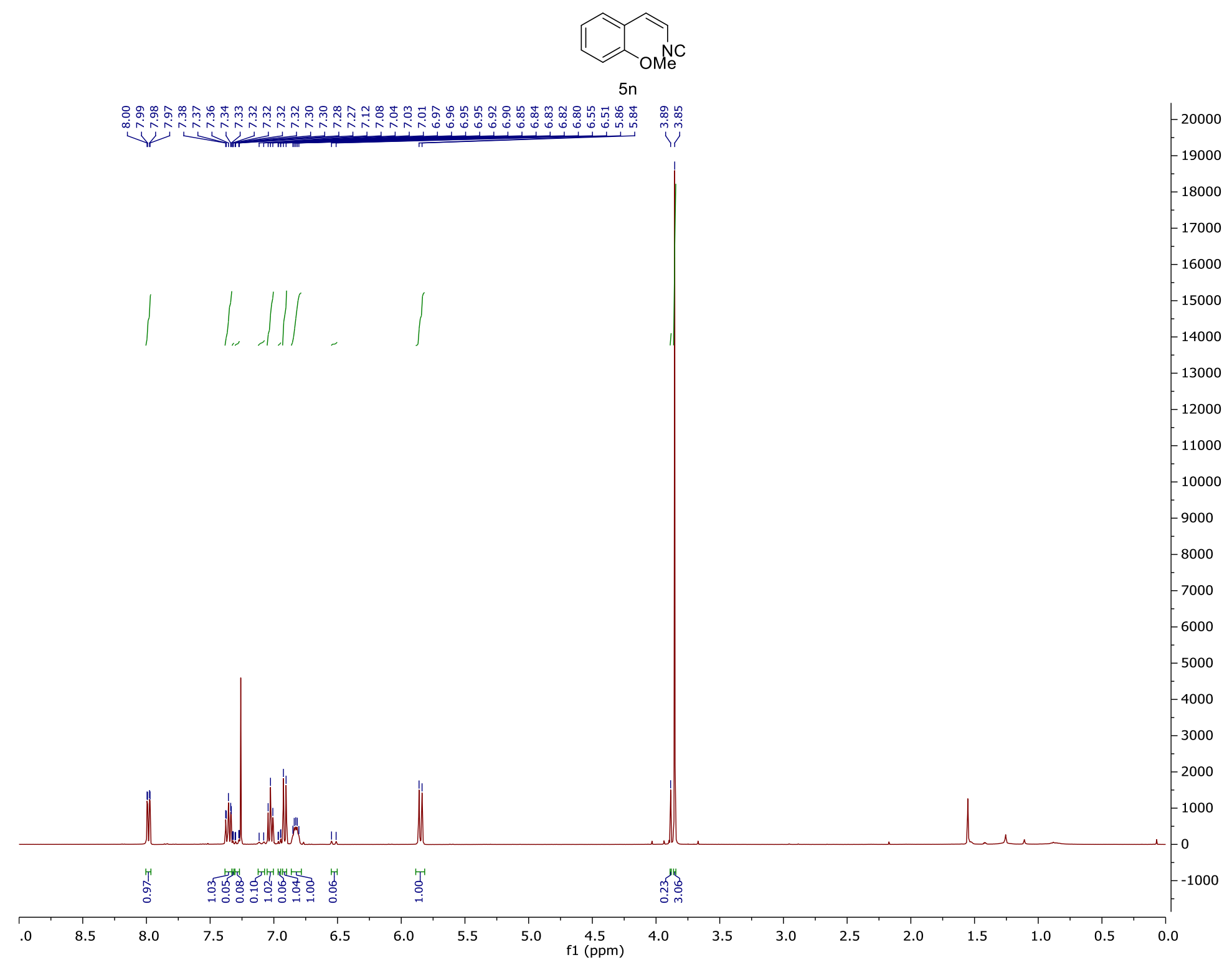




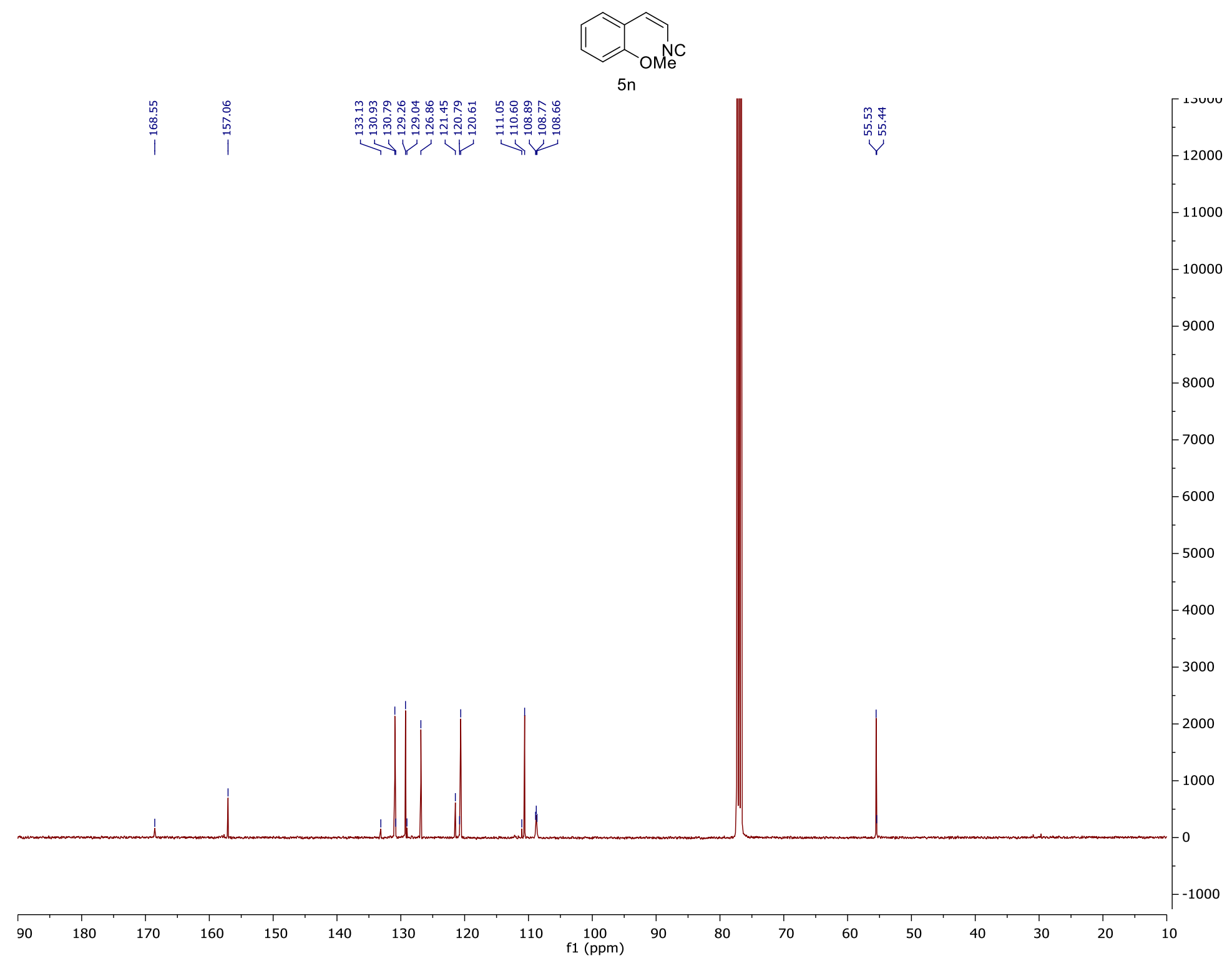




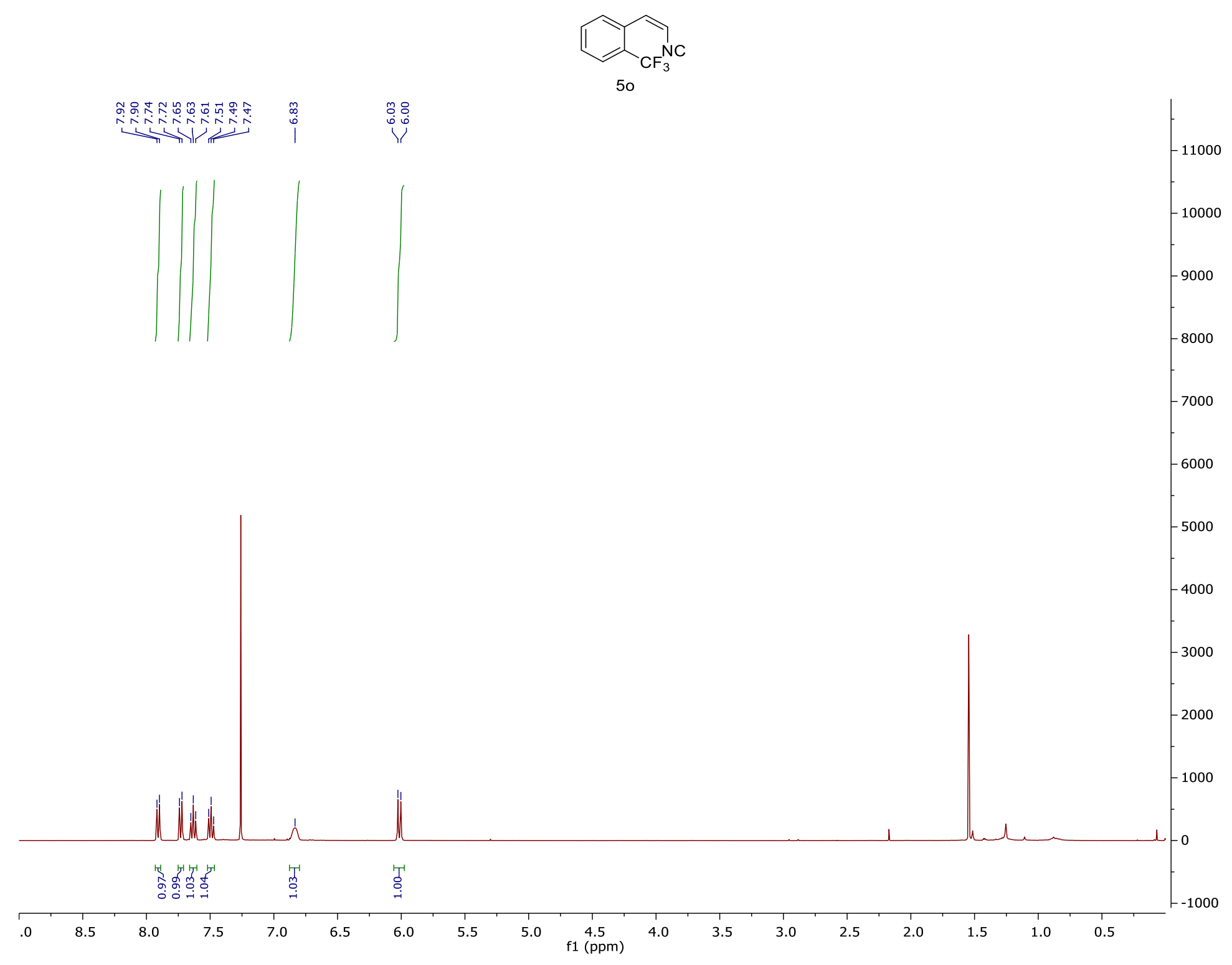




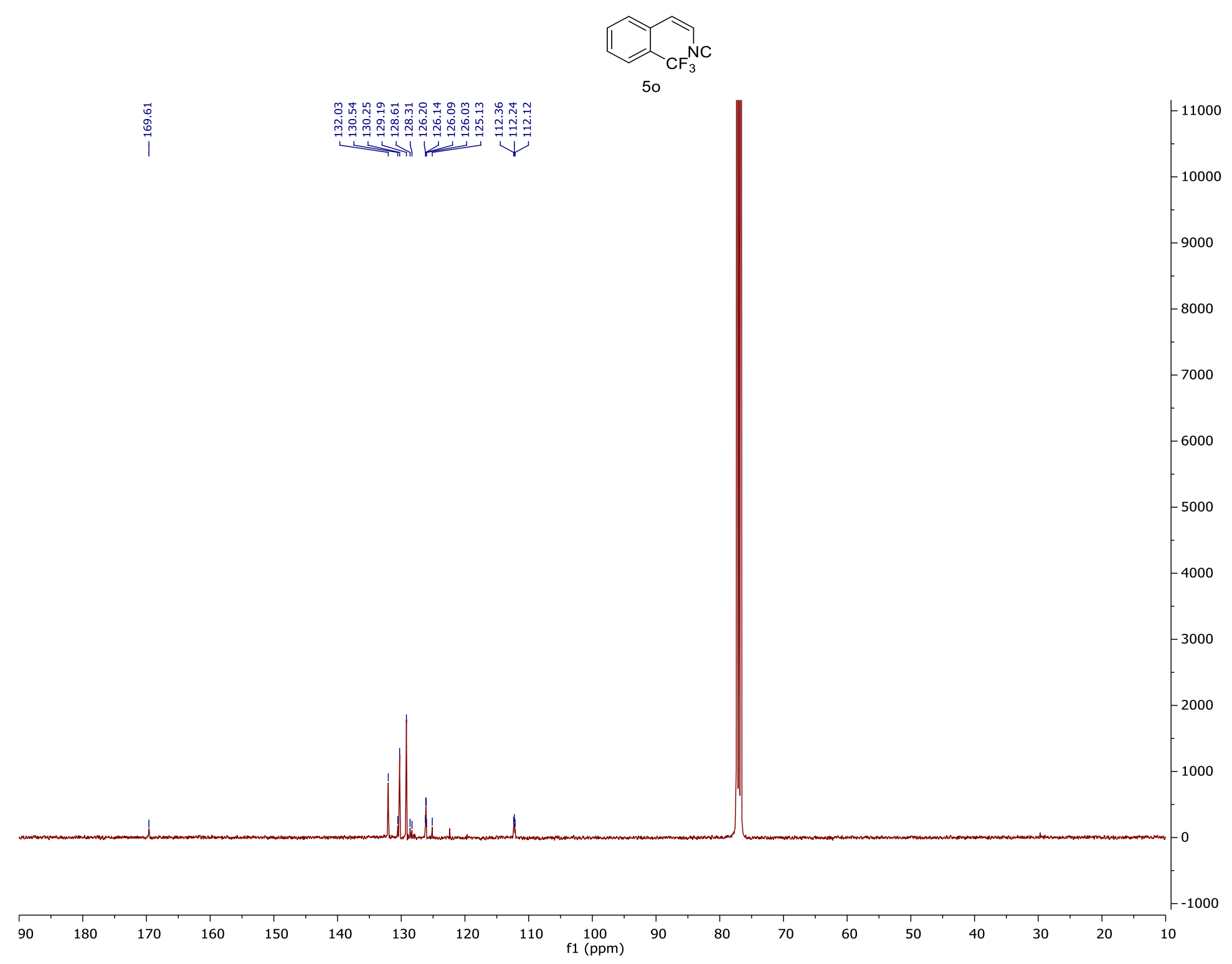




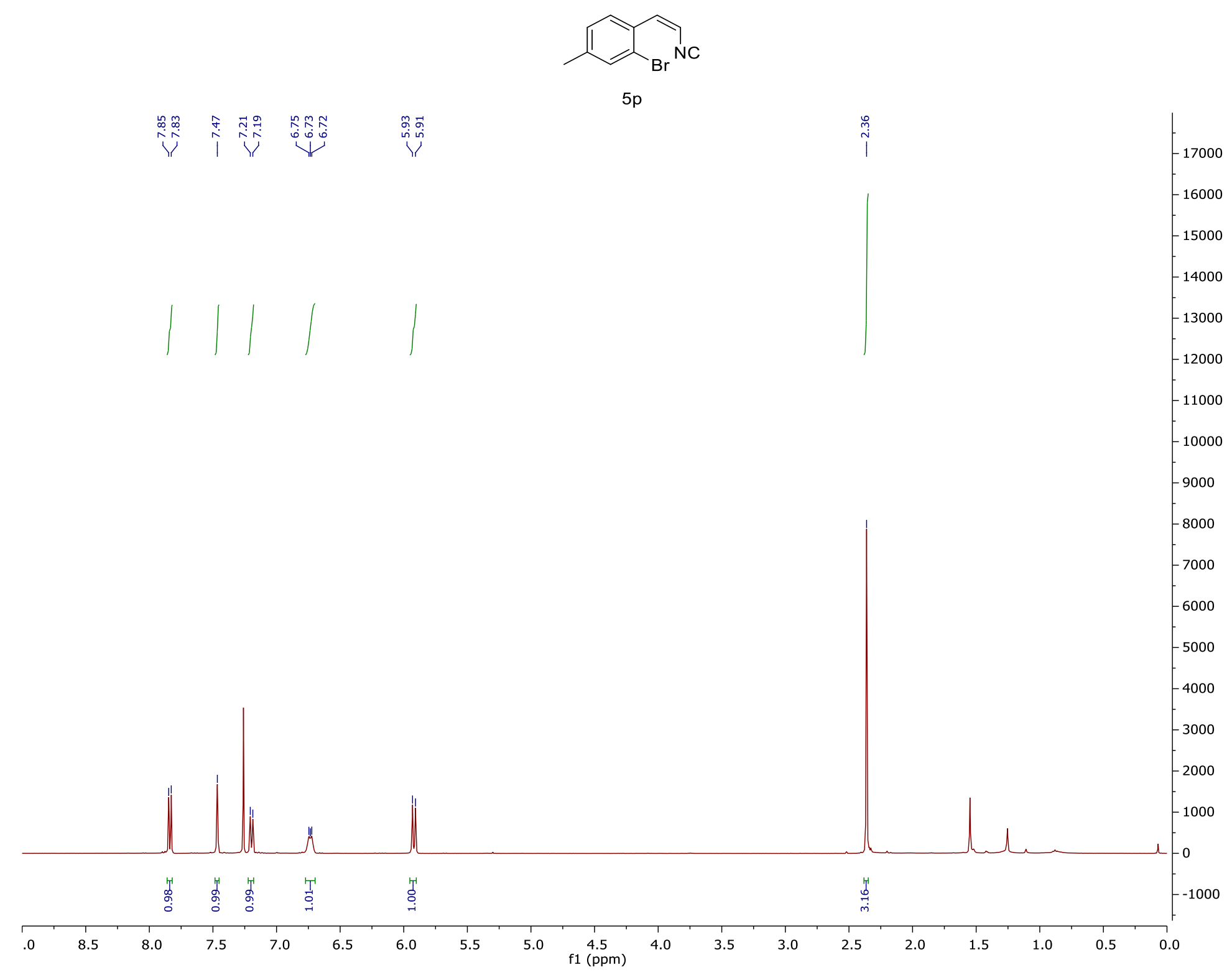




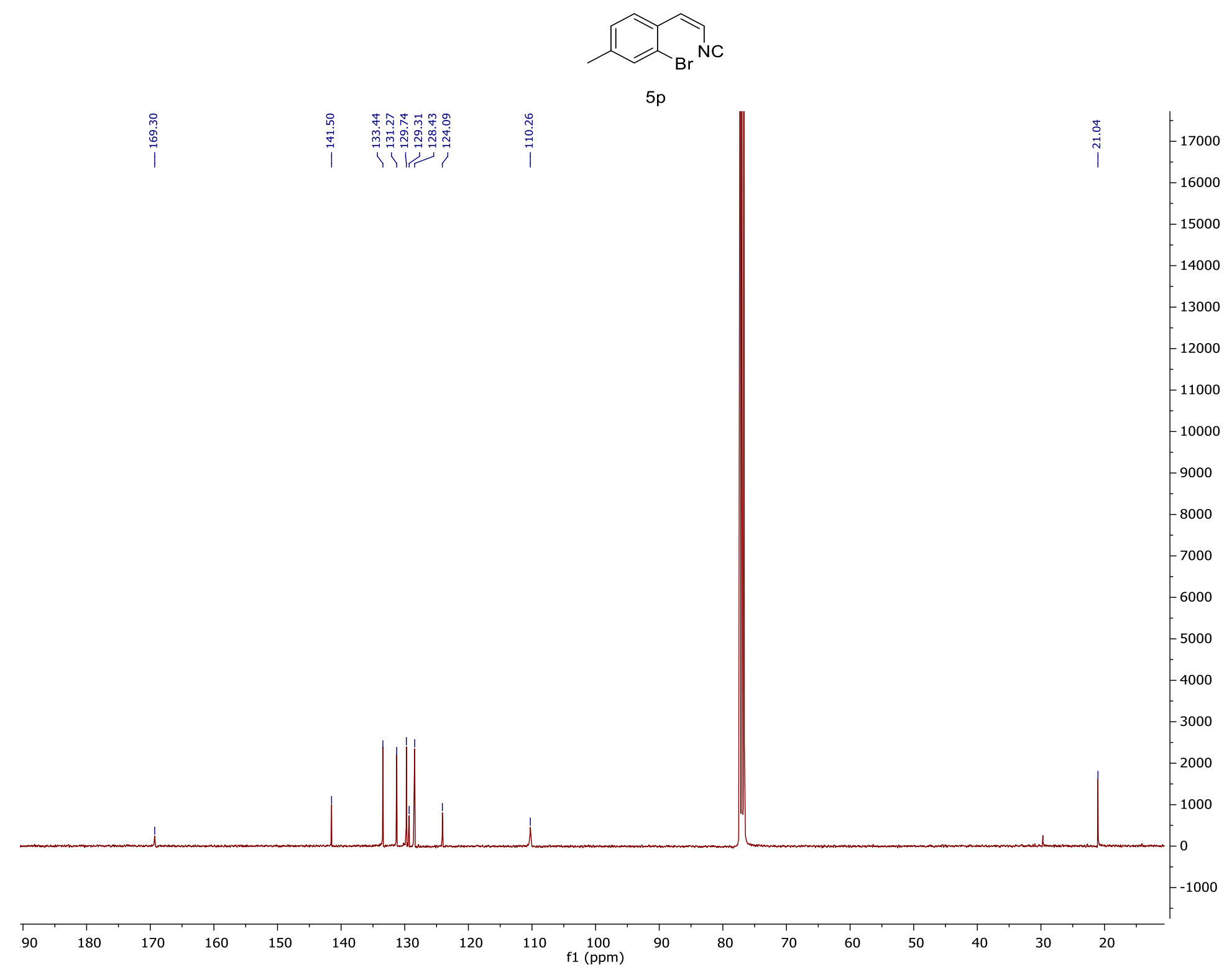




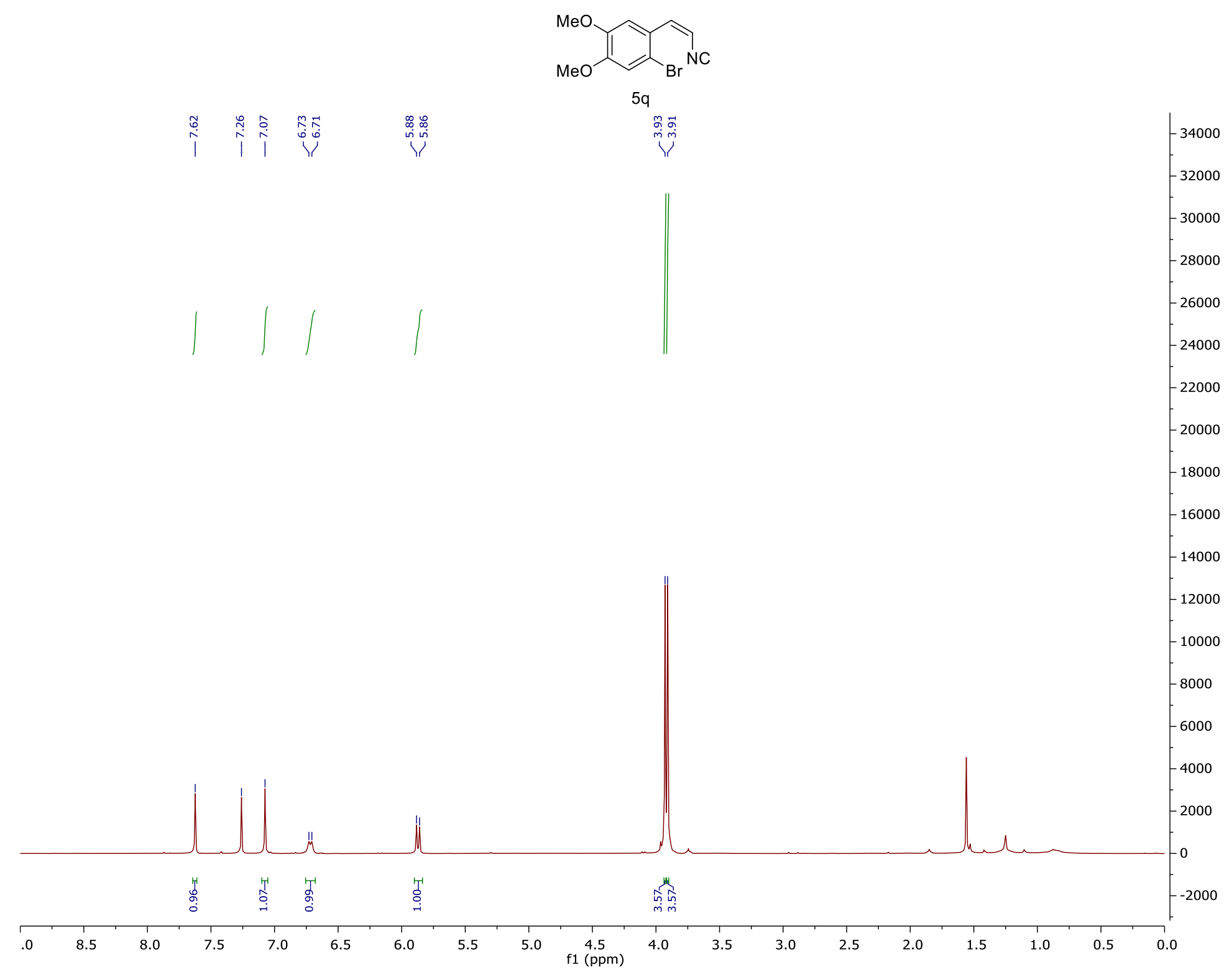




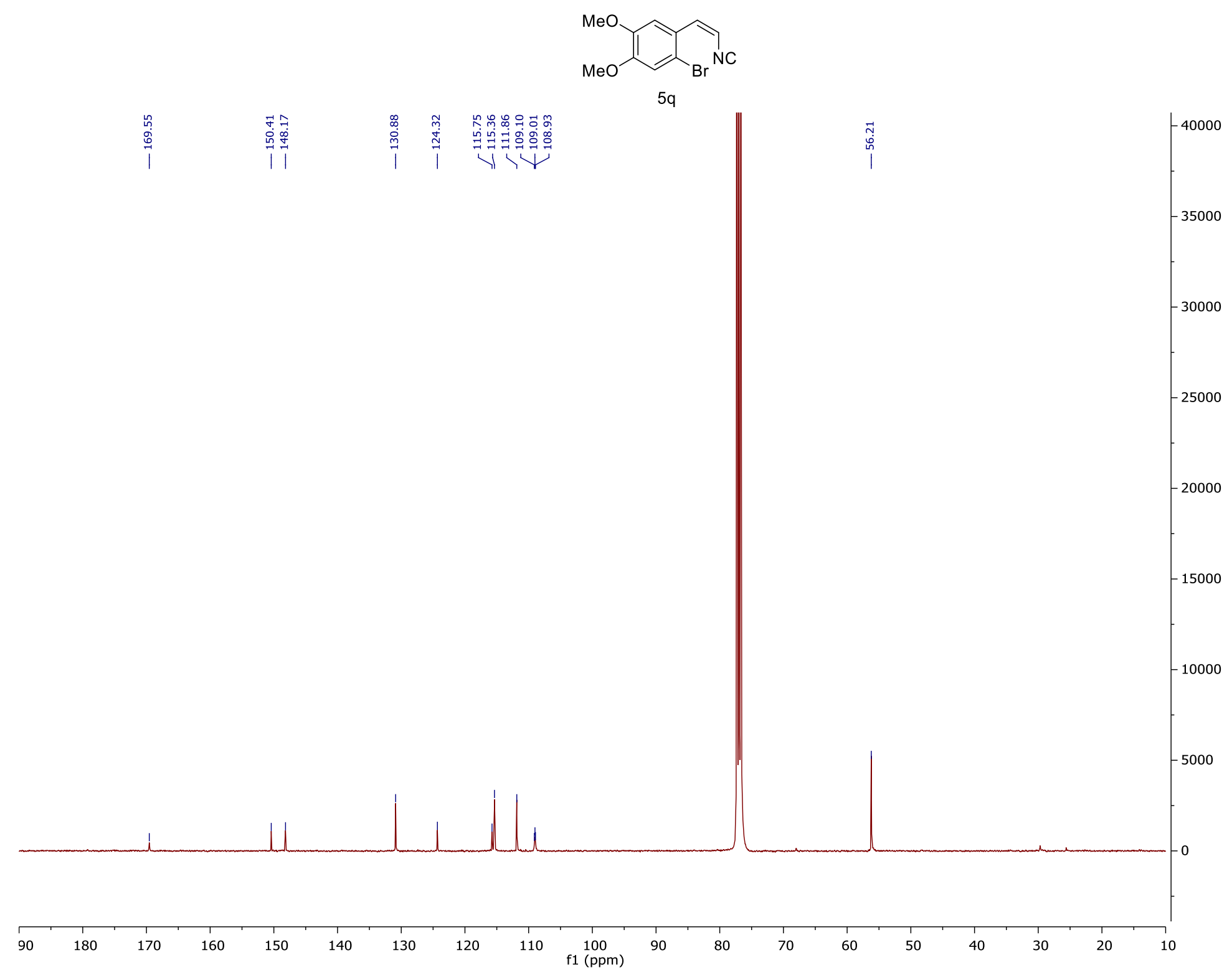




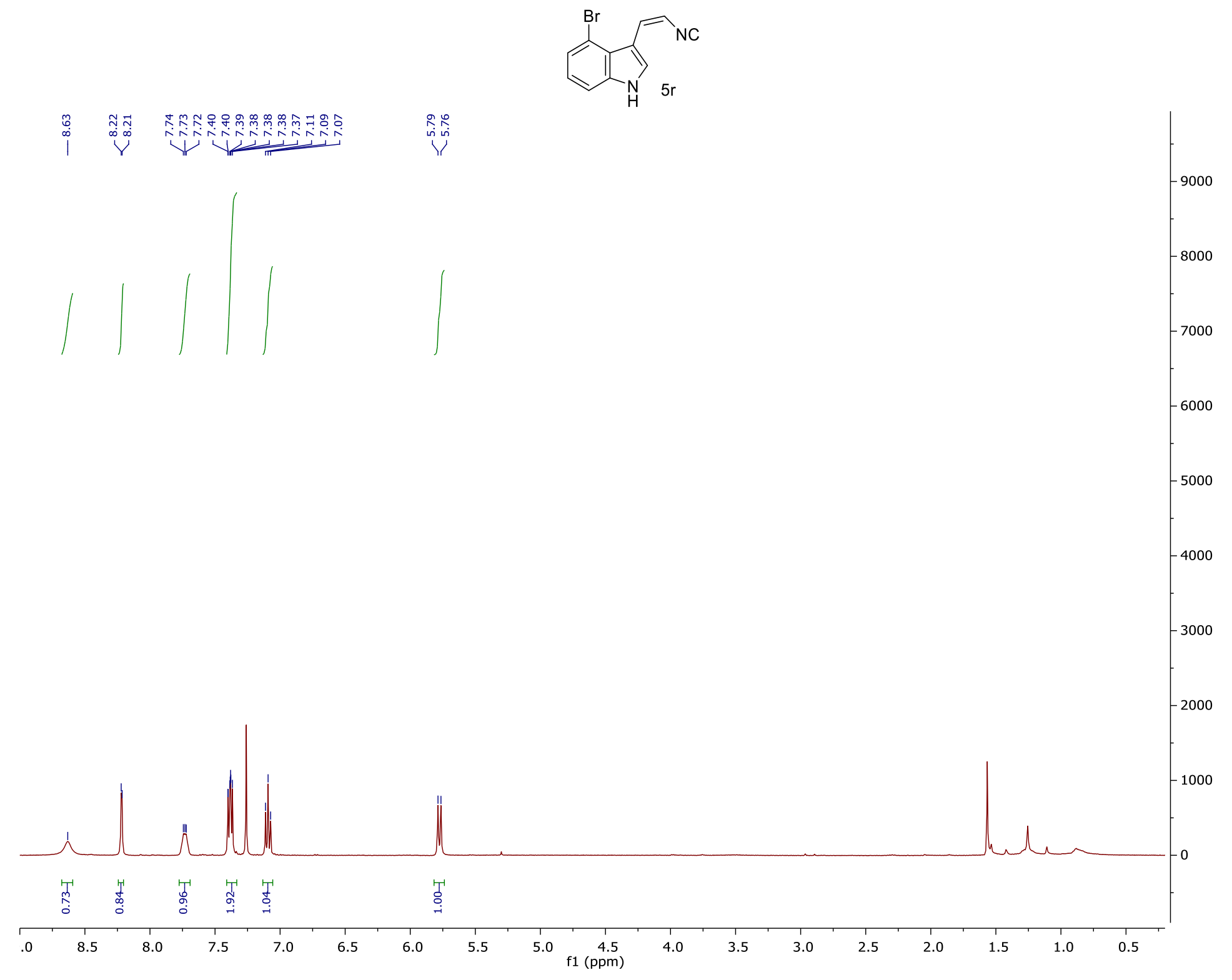




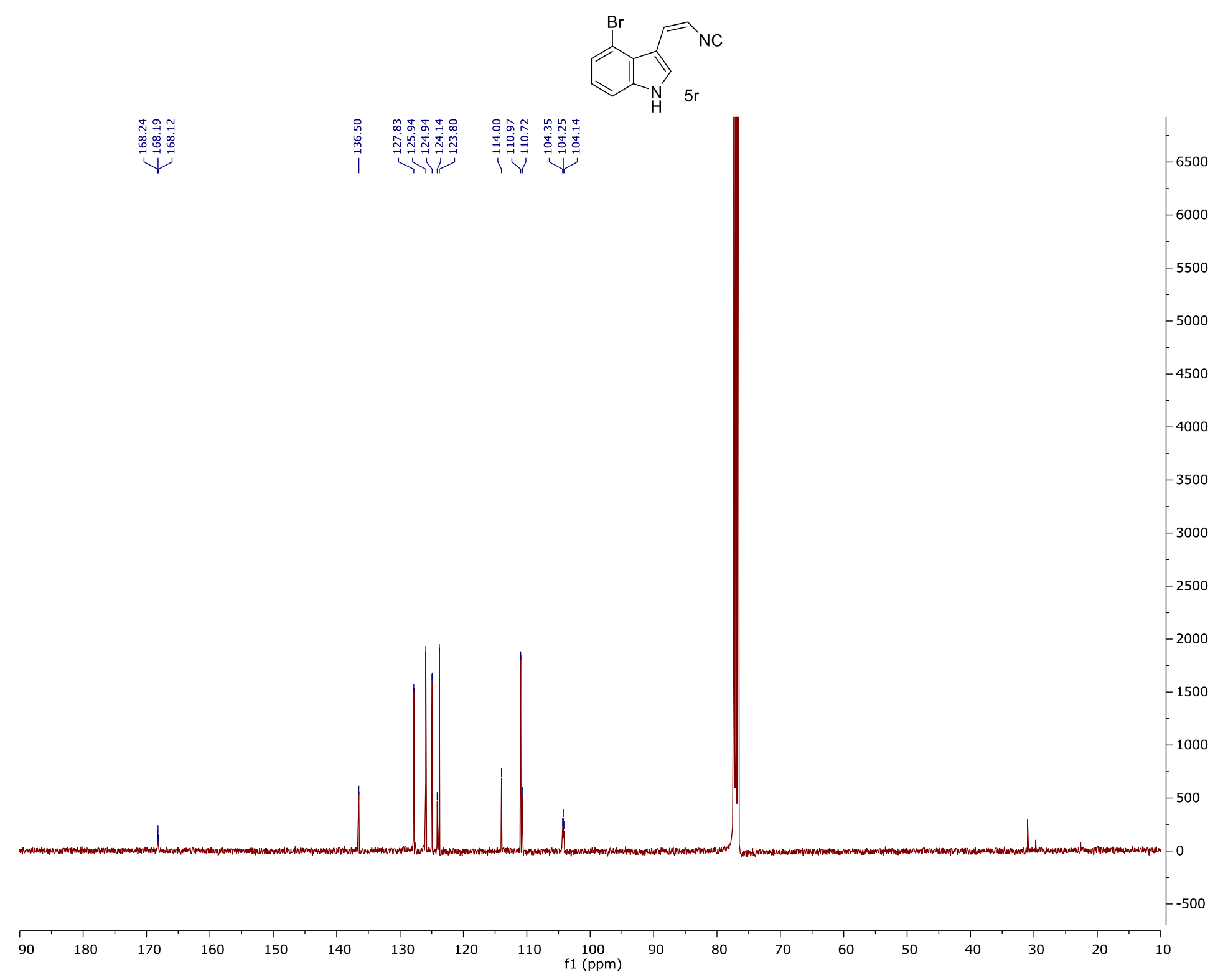




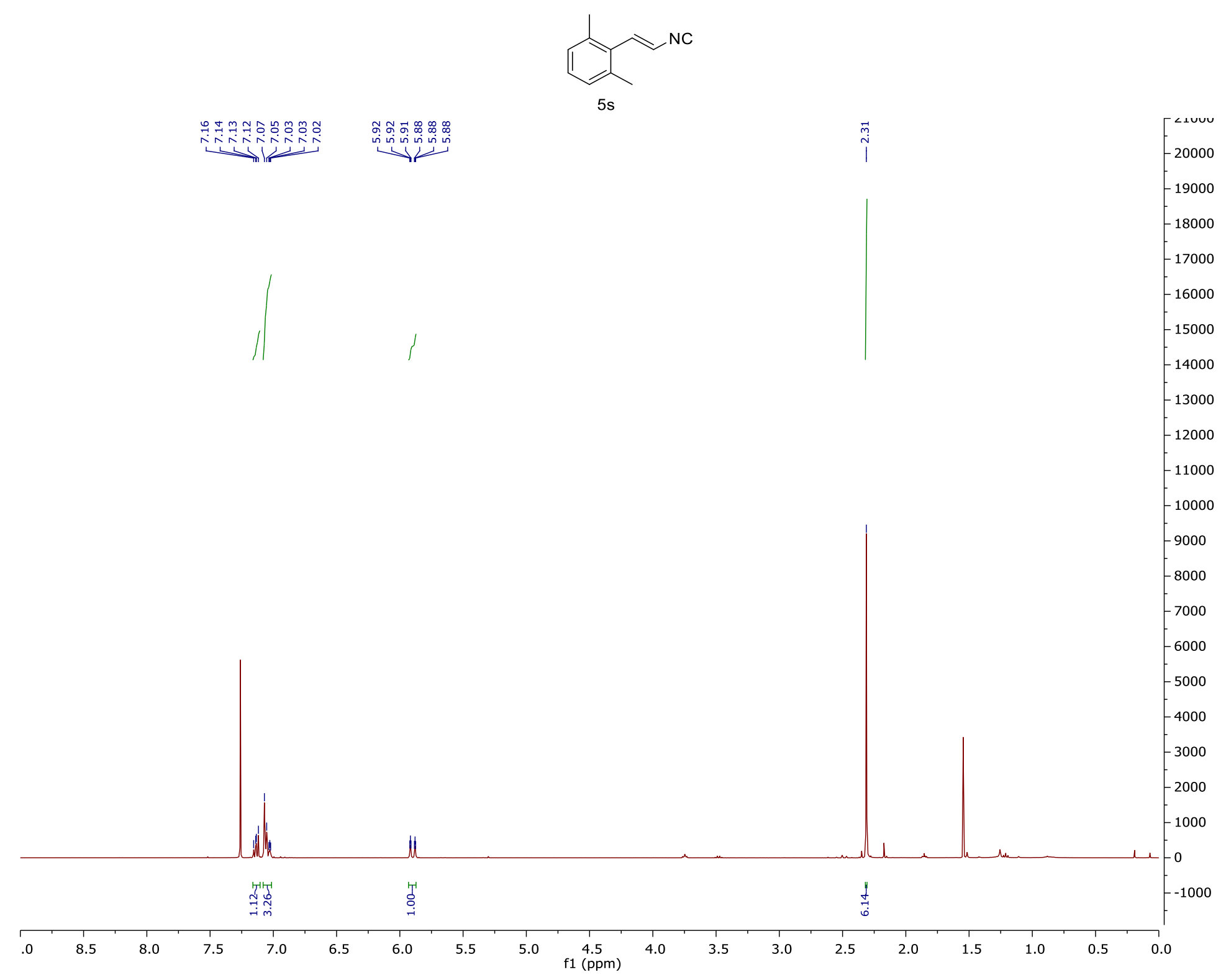




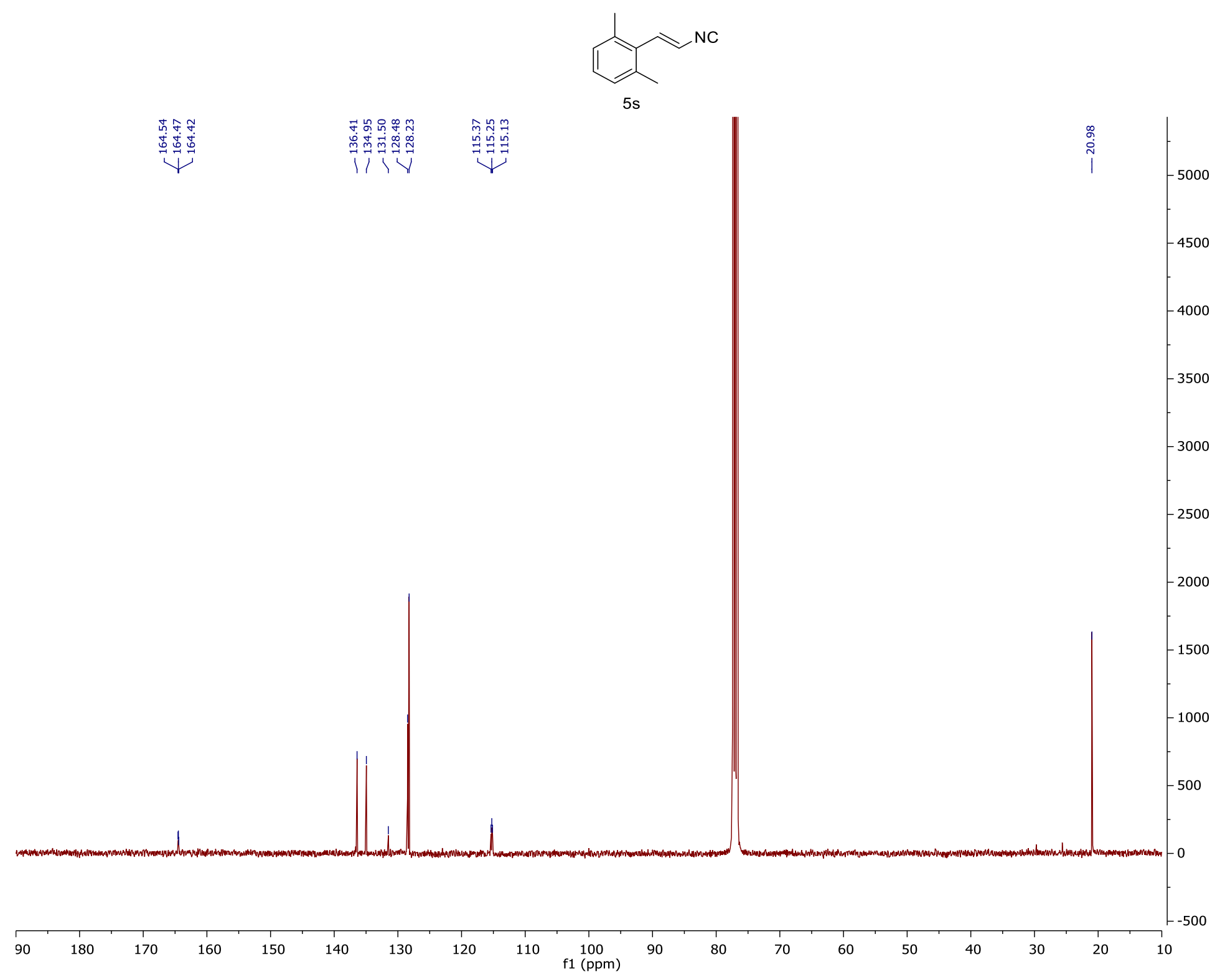




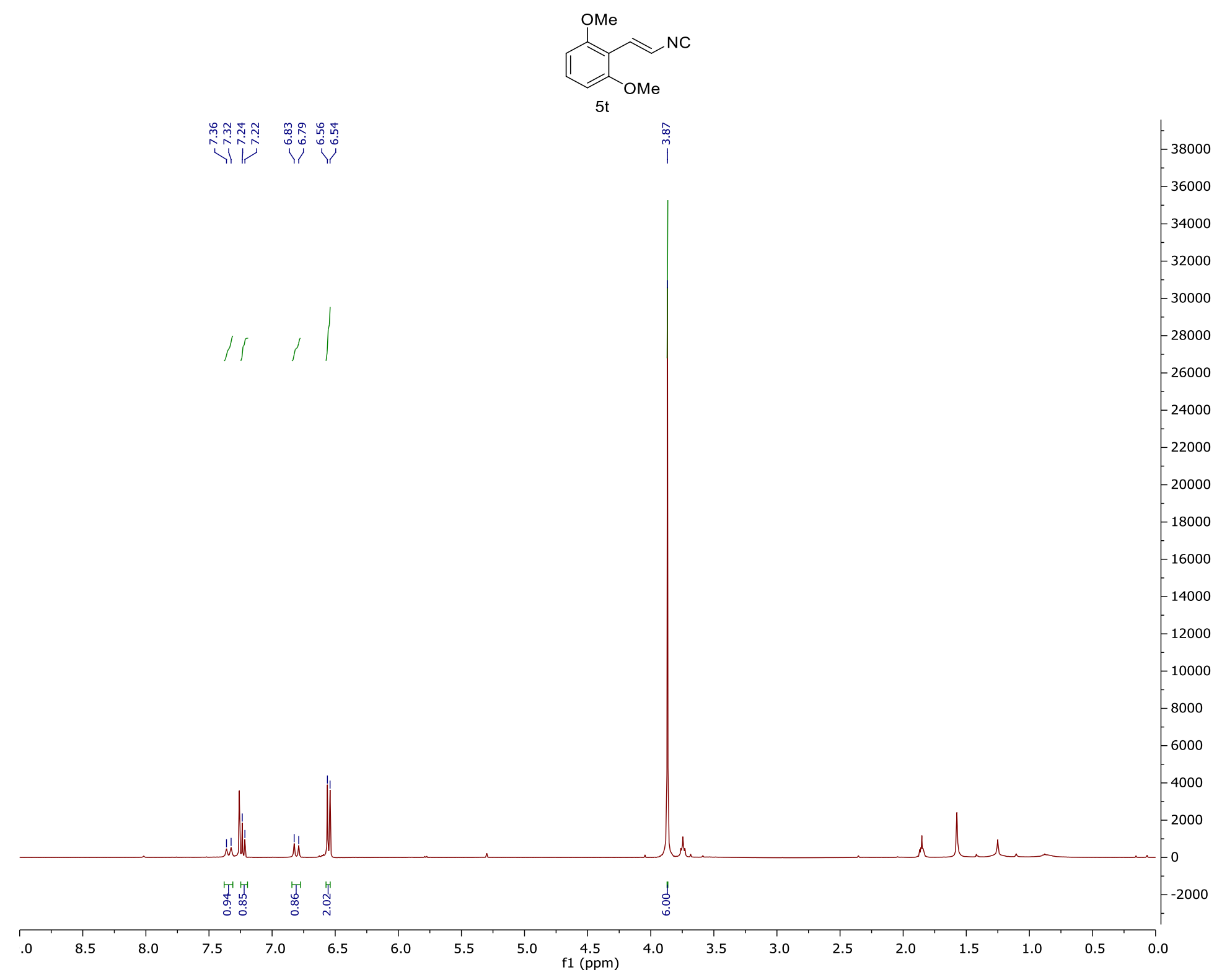




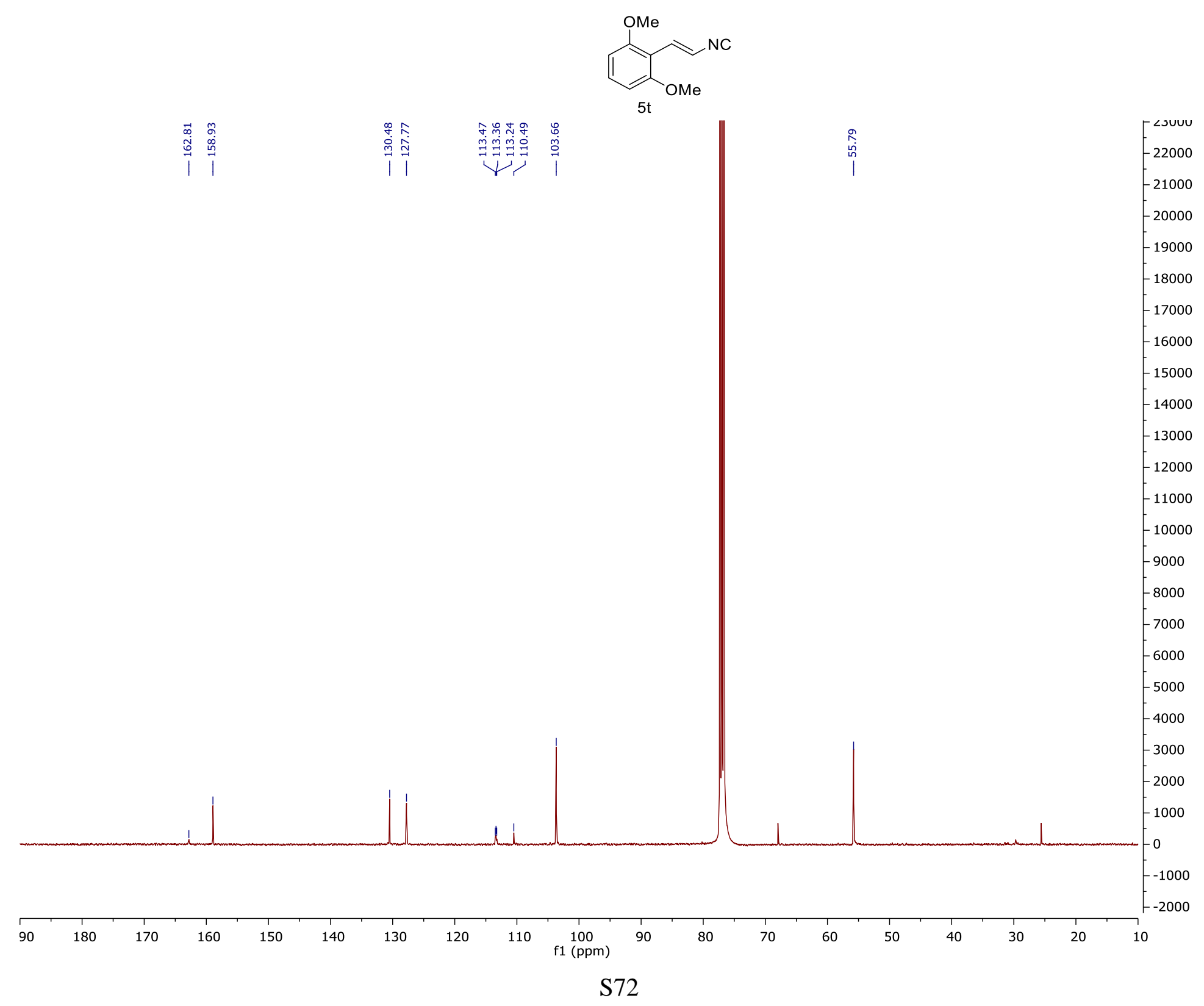




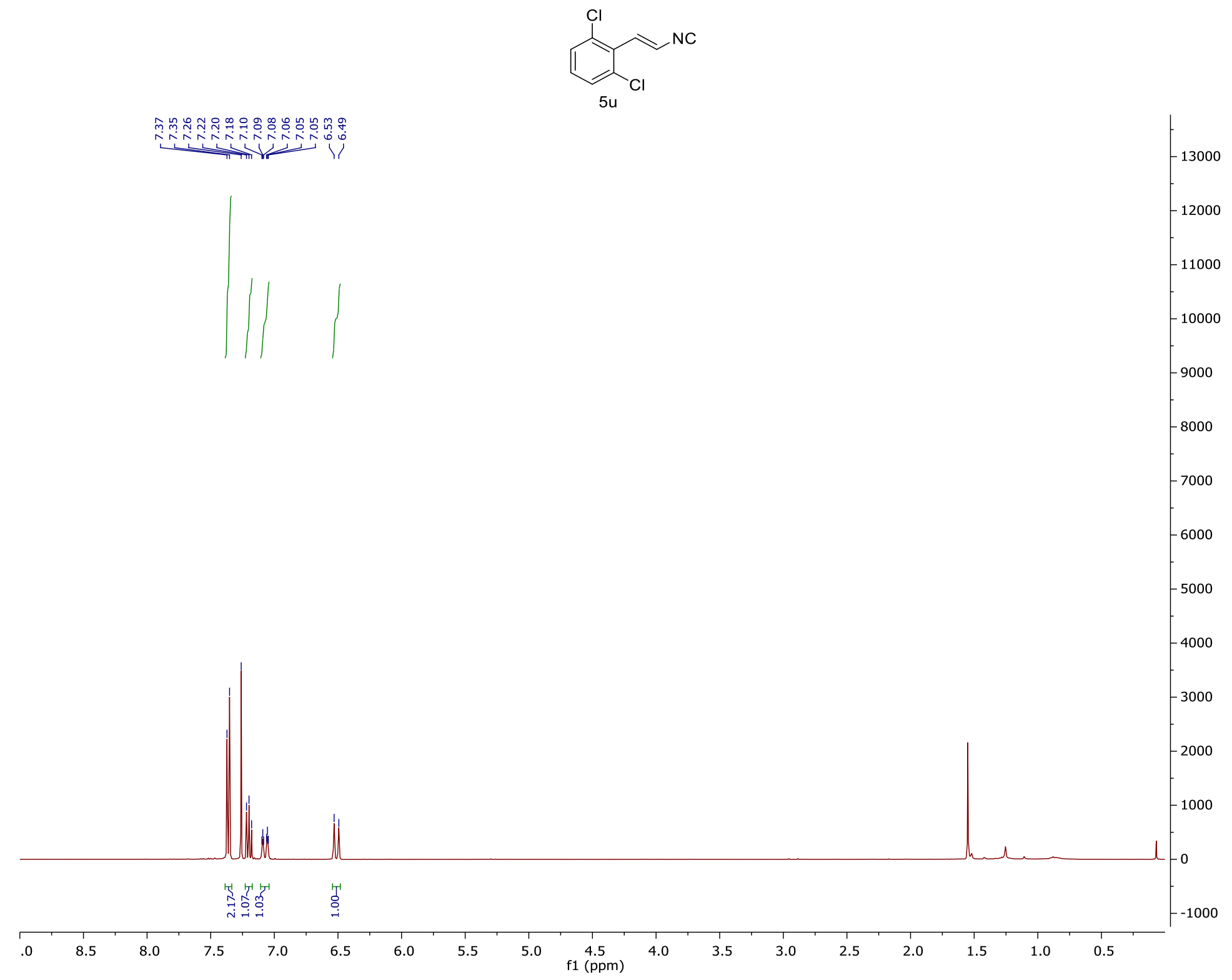




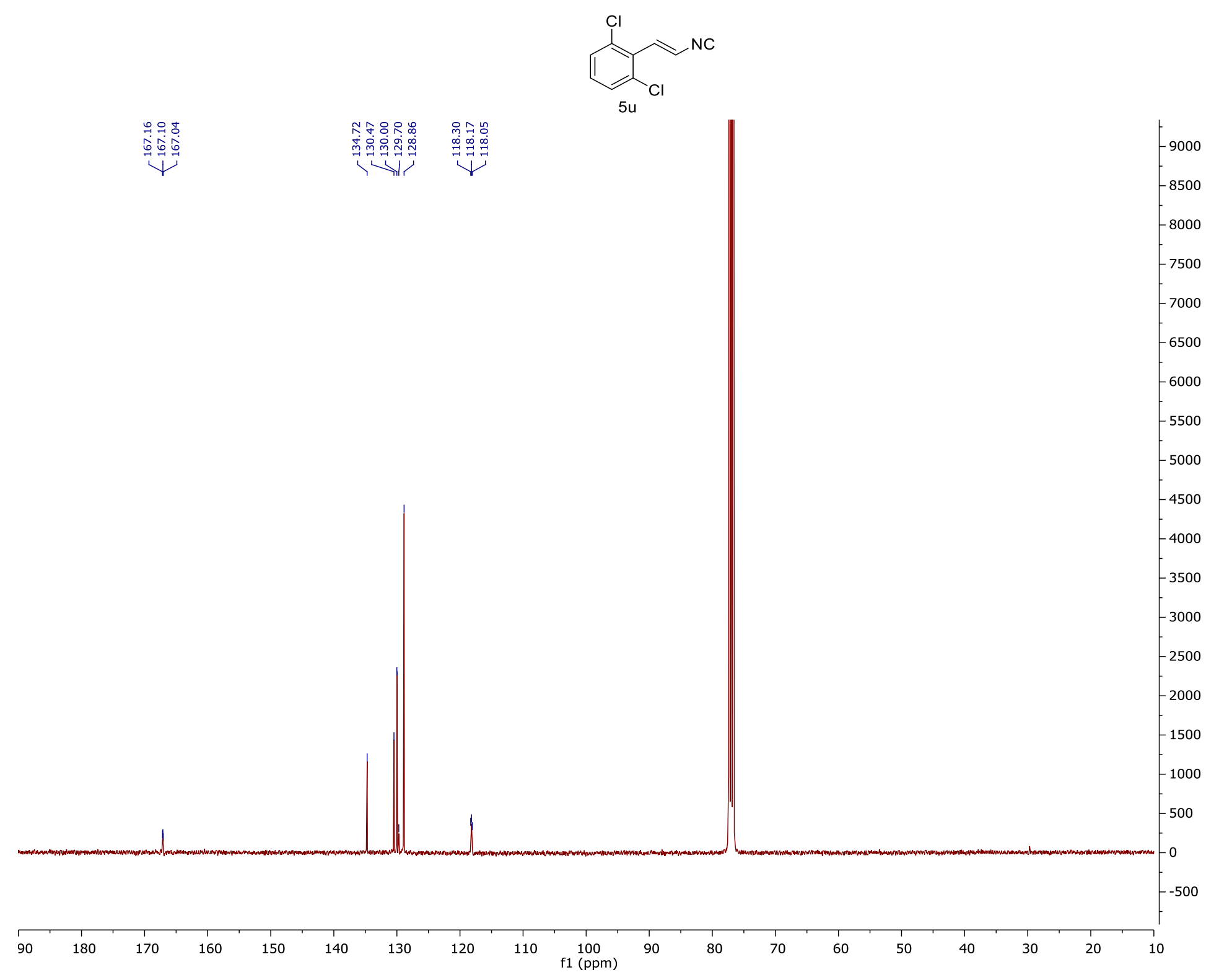




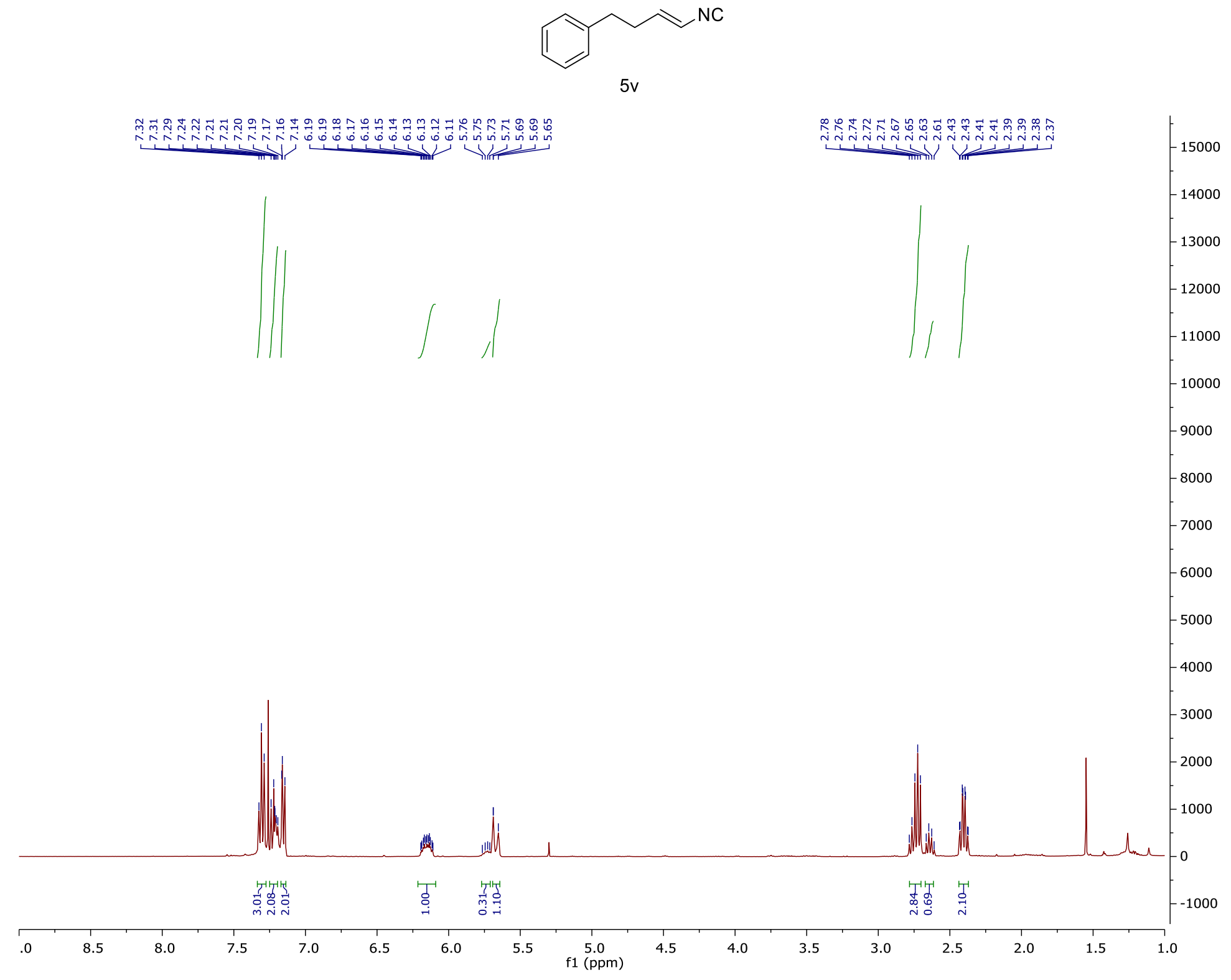




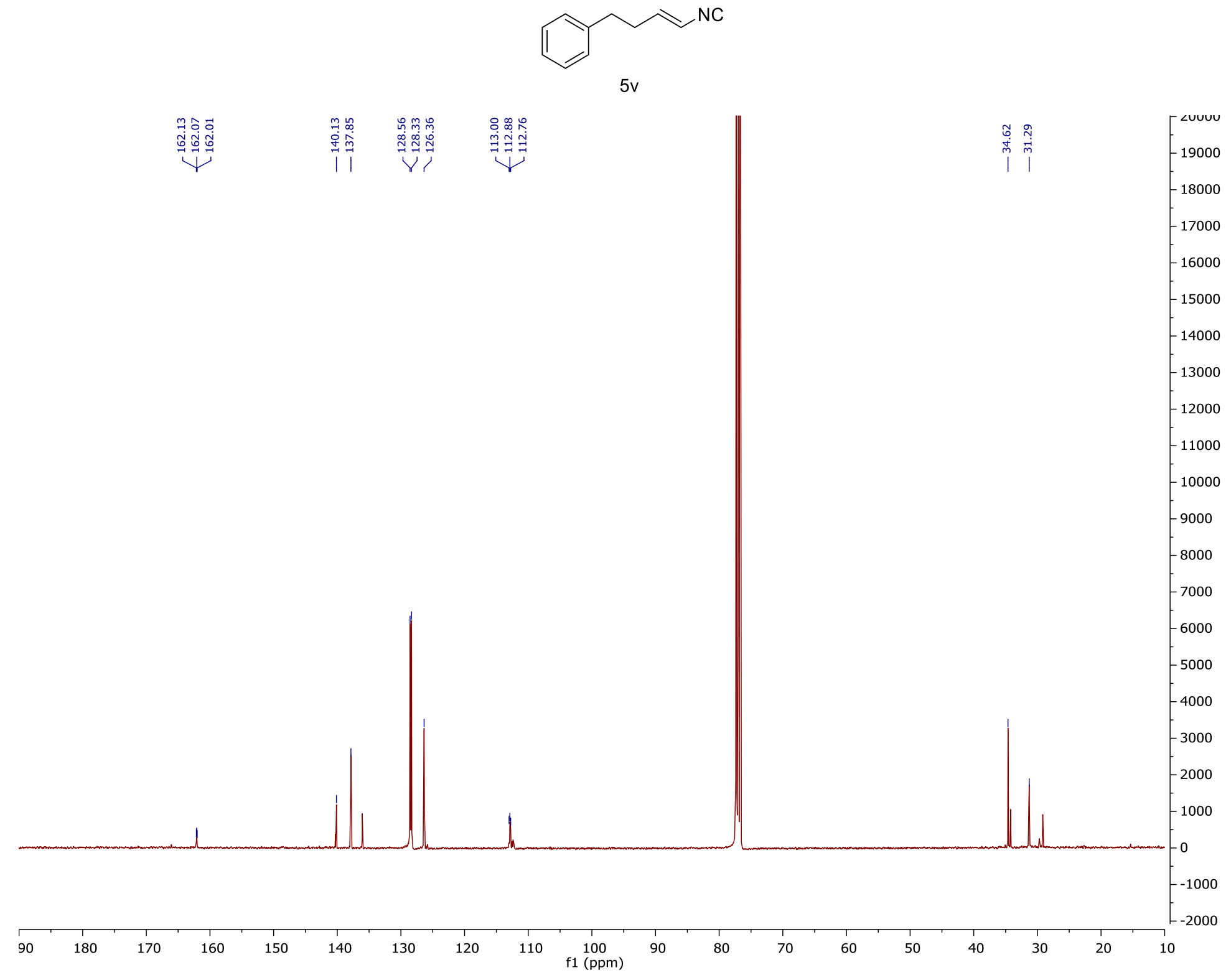




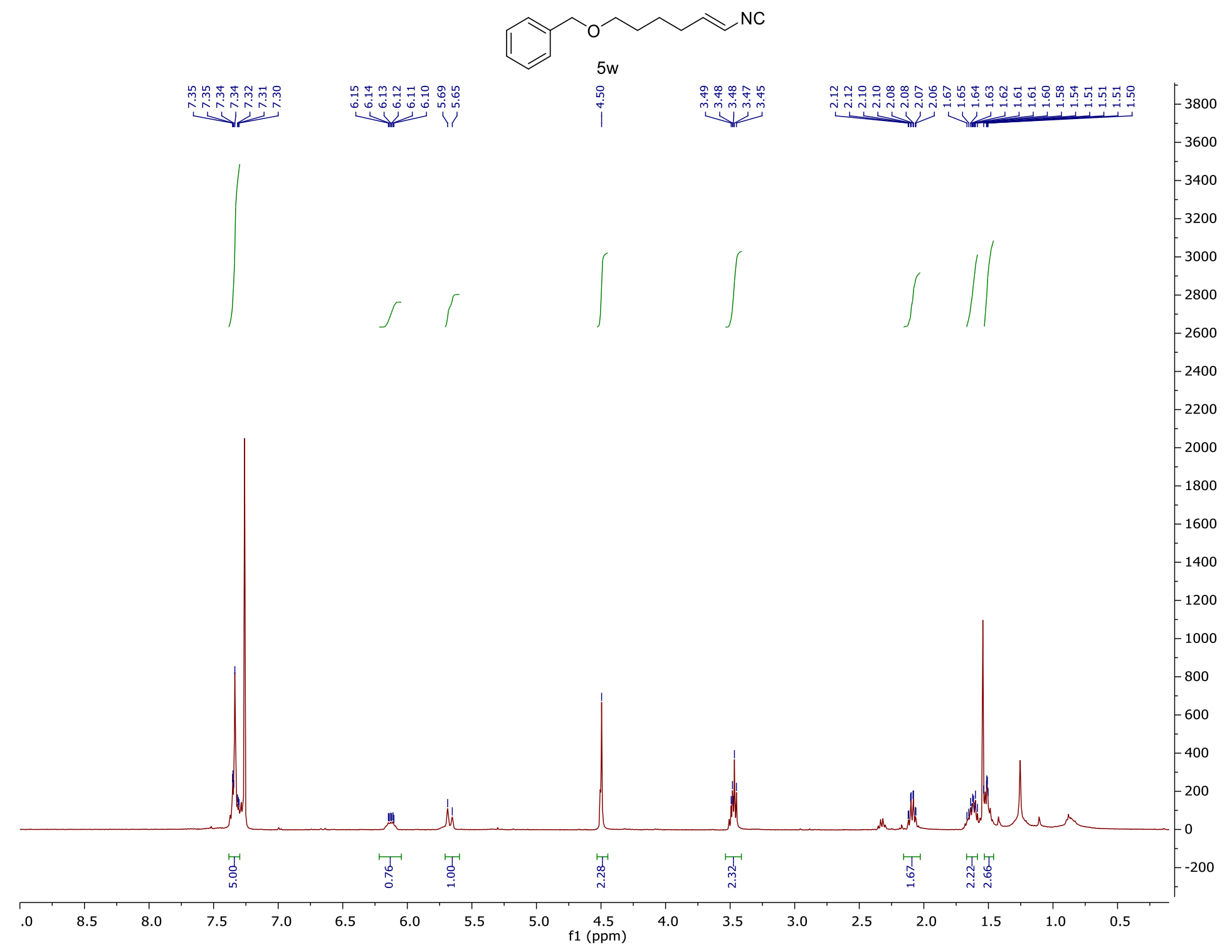




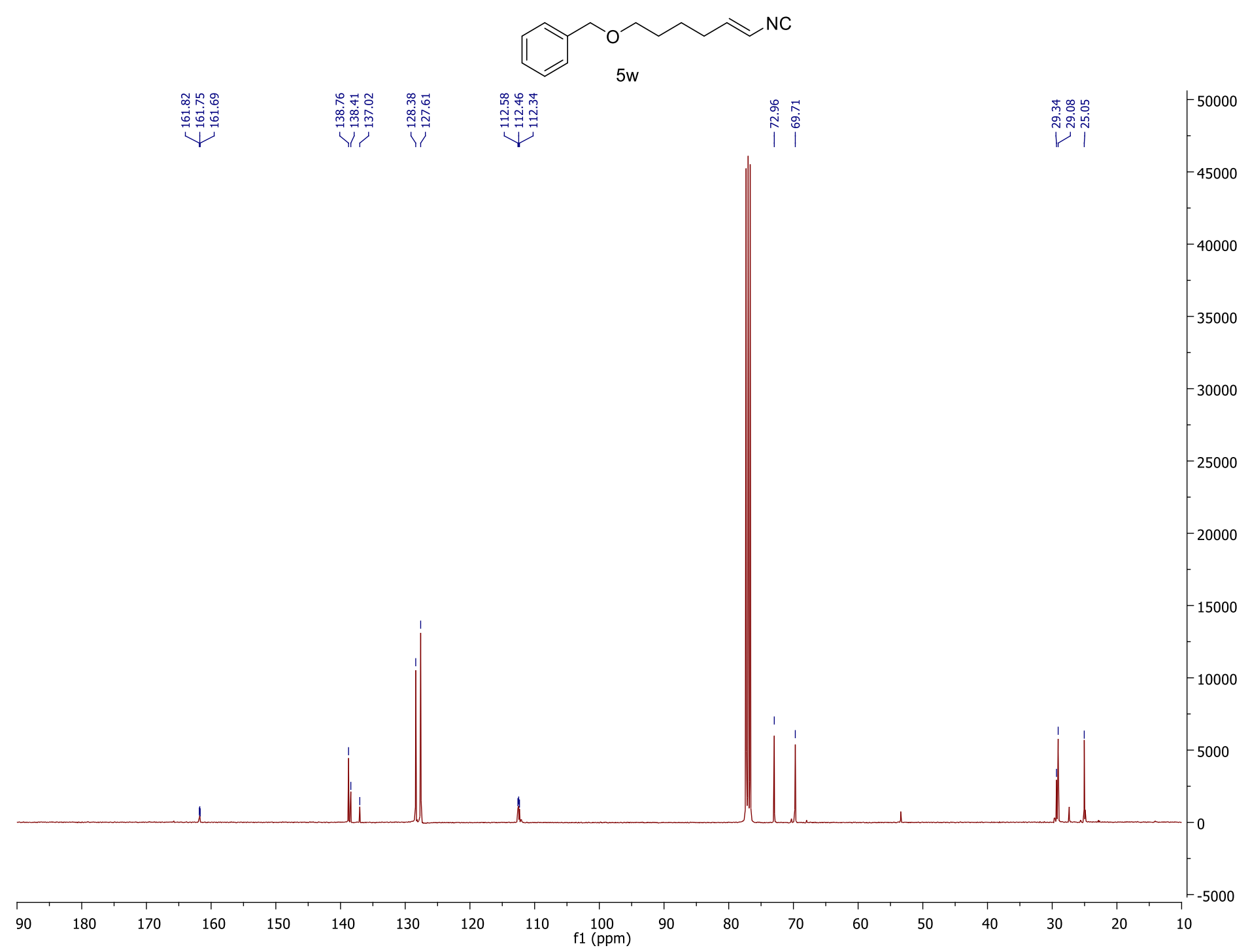




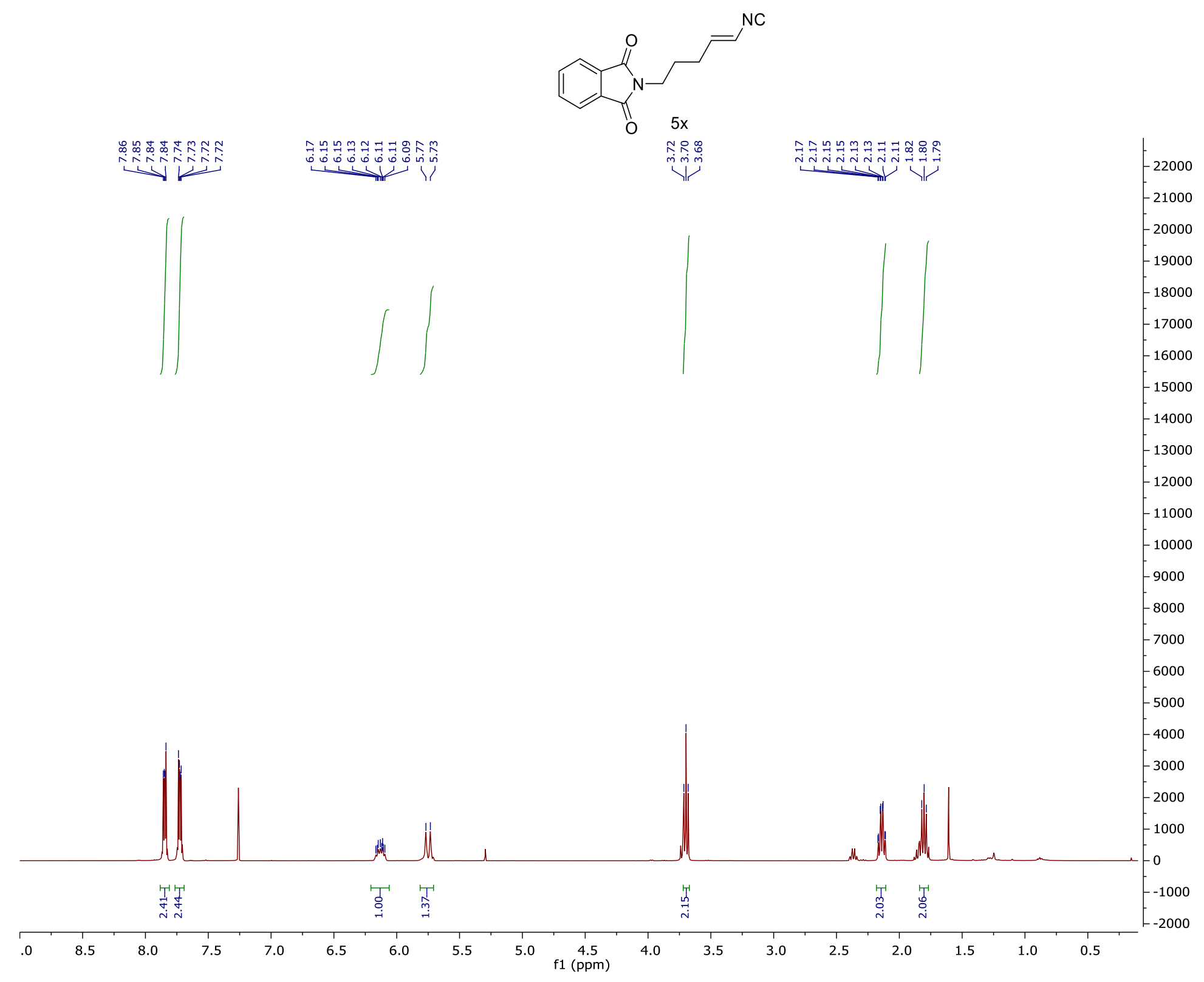

S79 


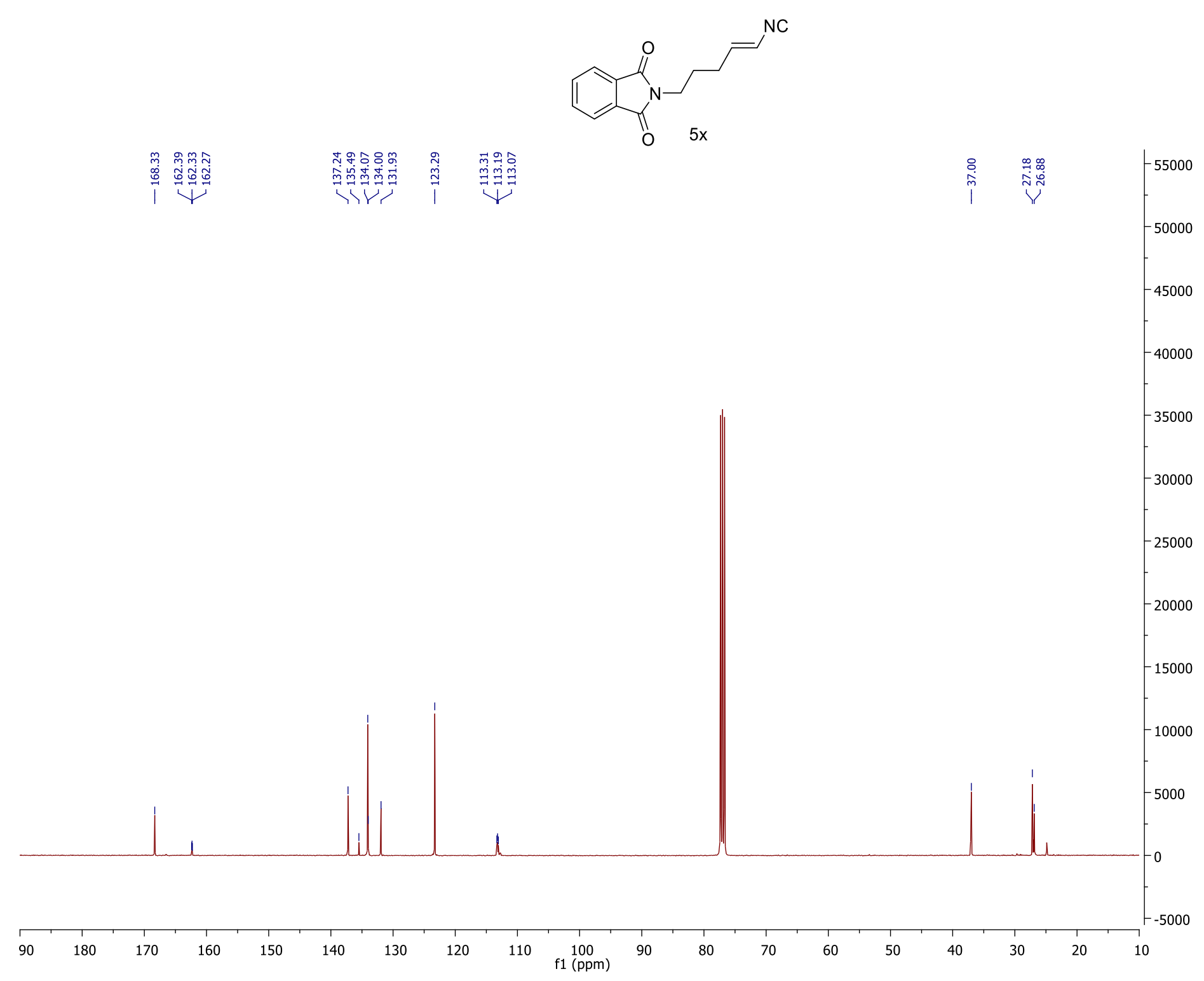




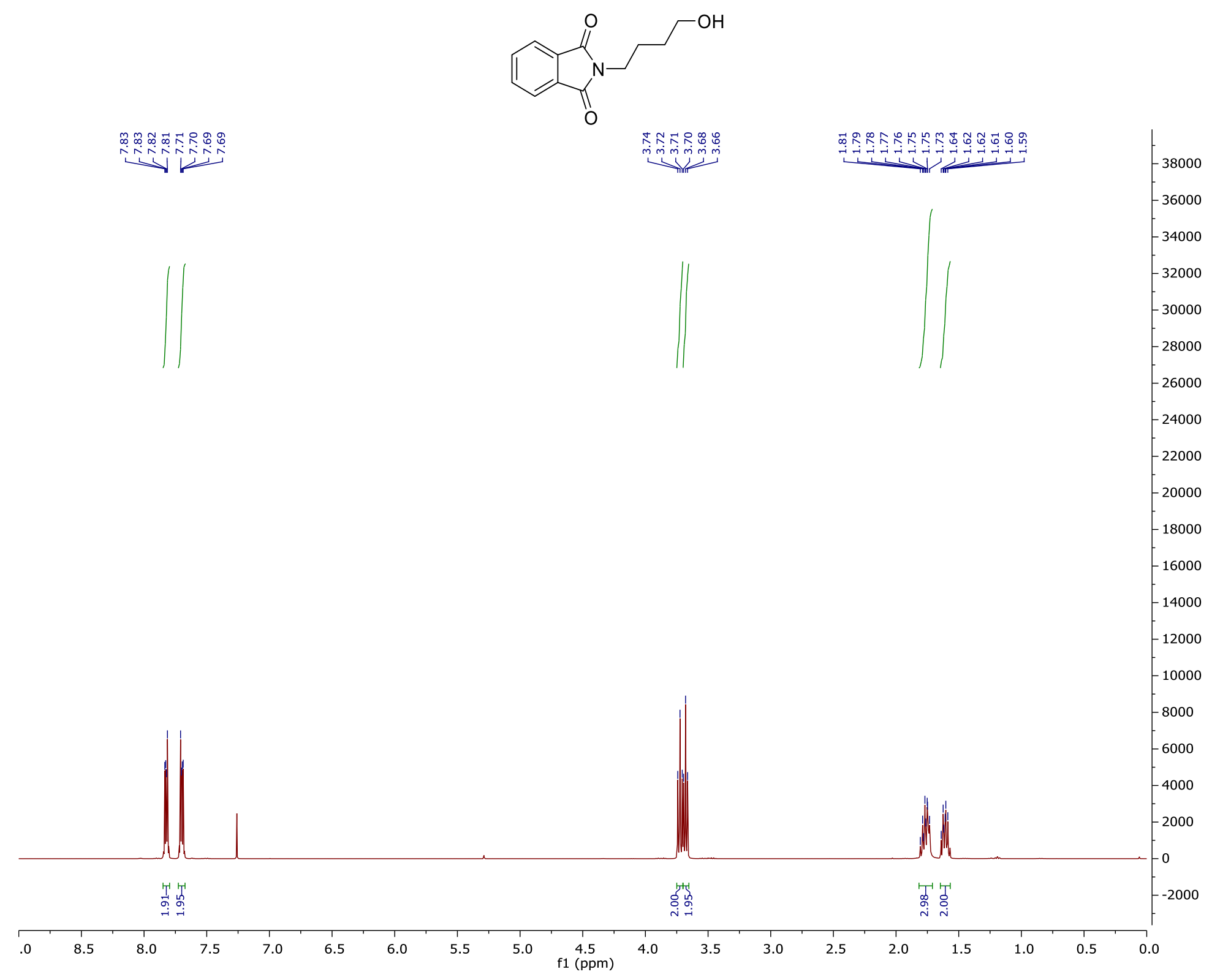




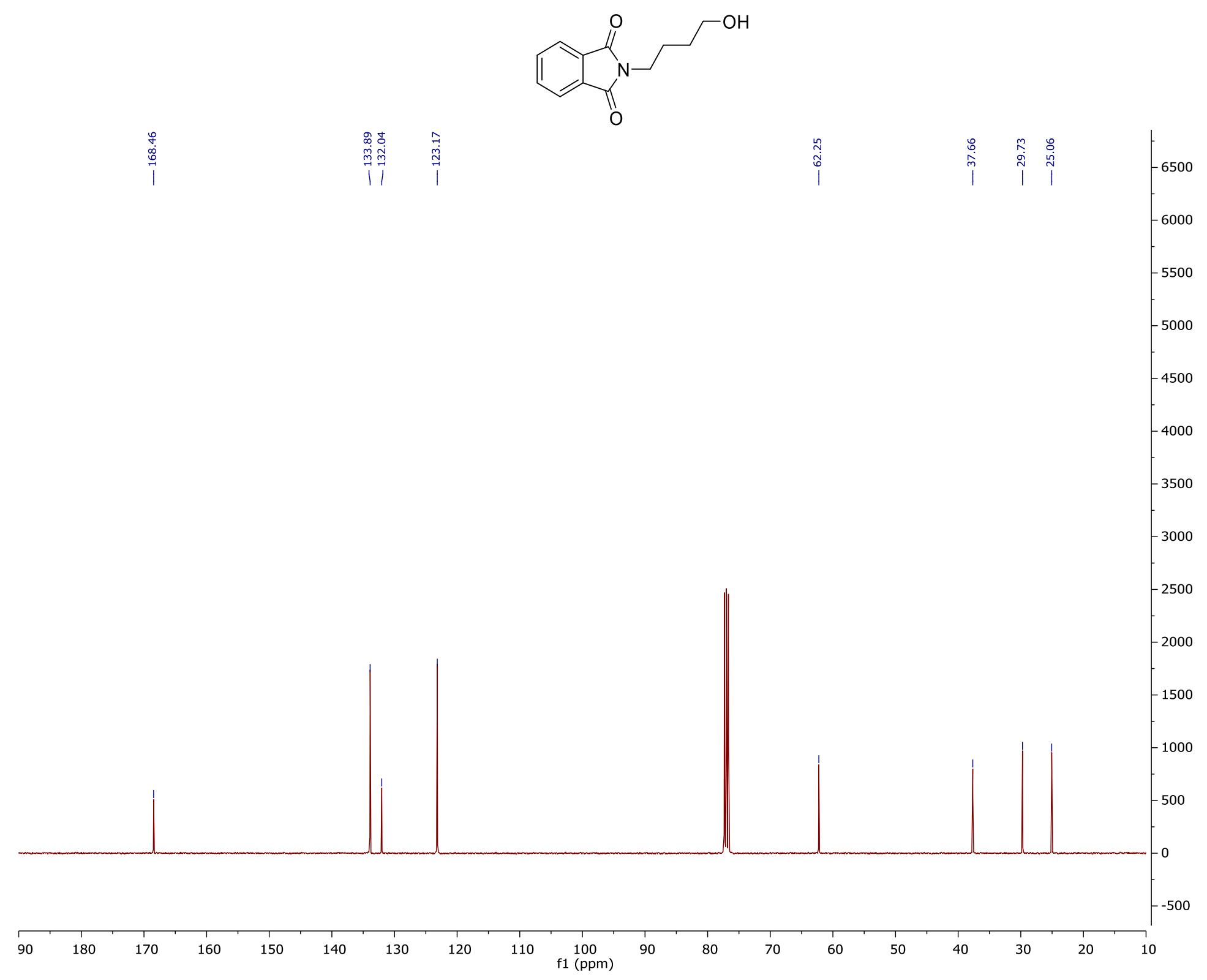




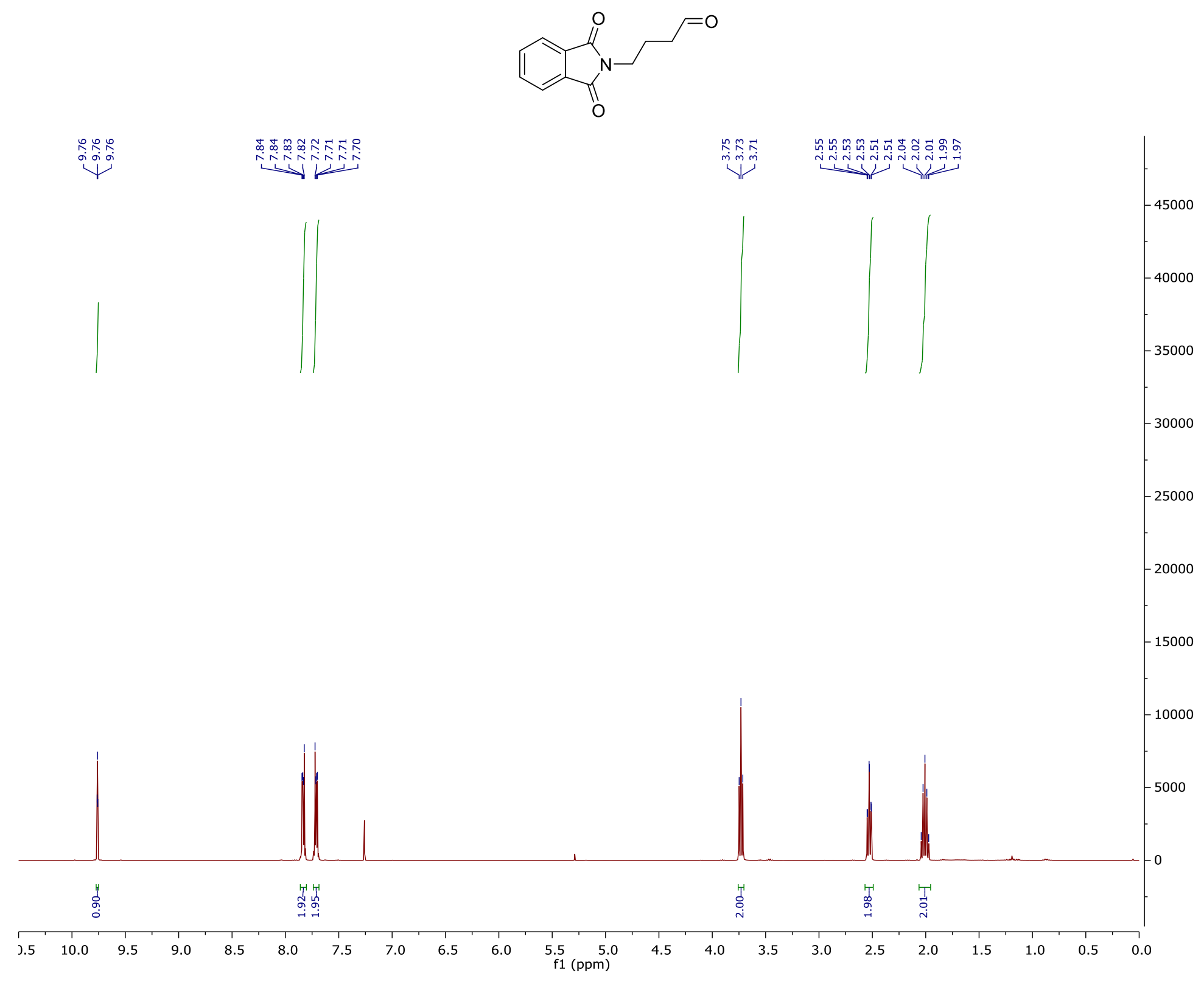




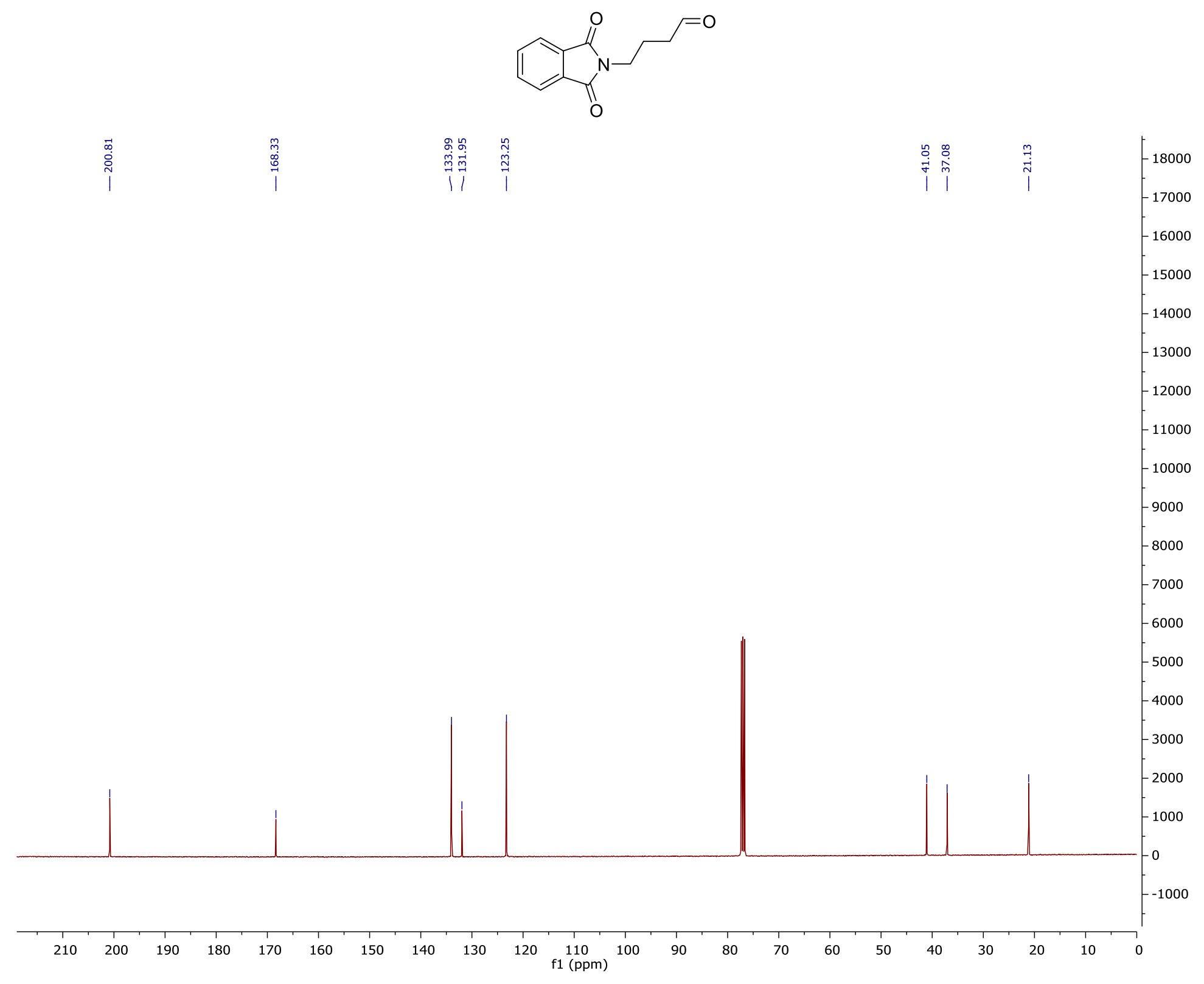




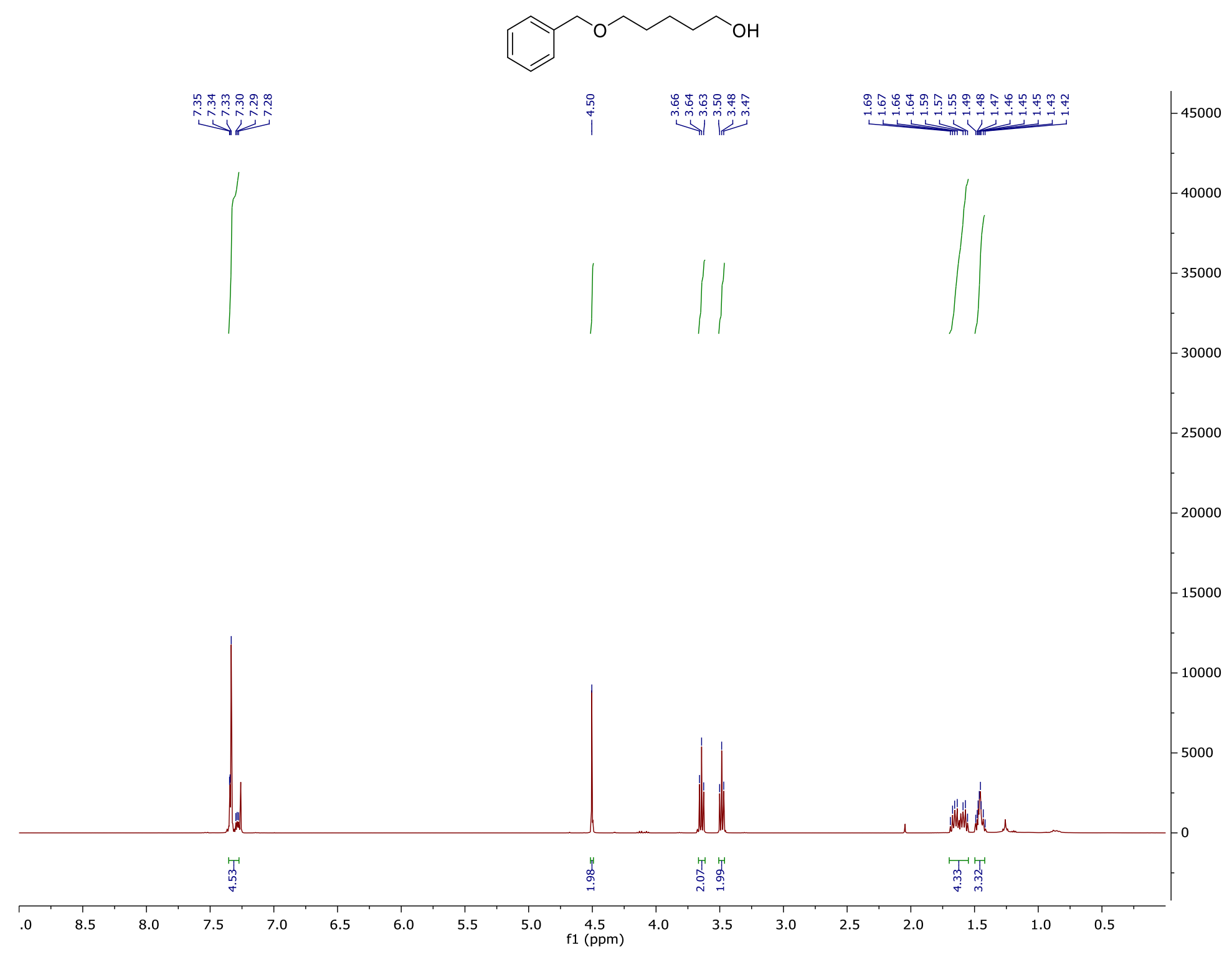




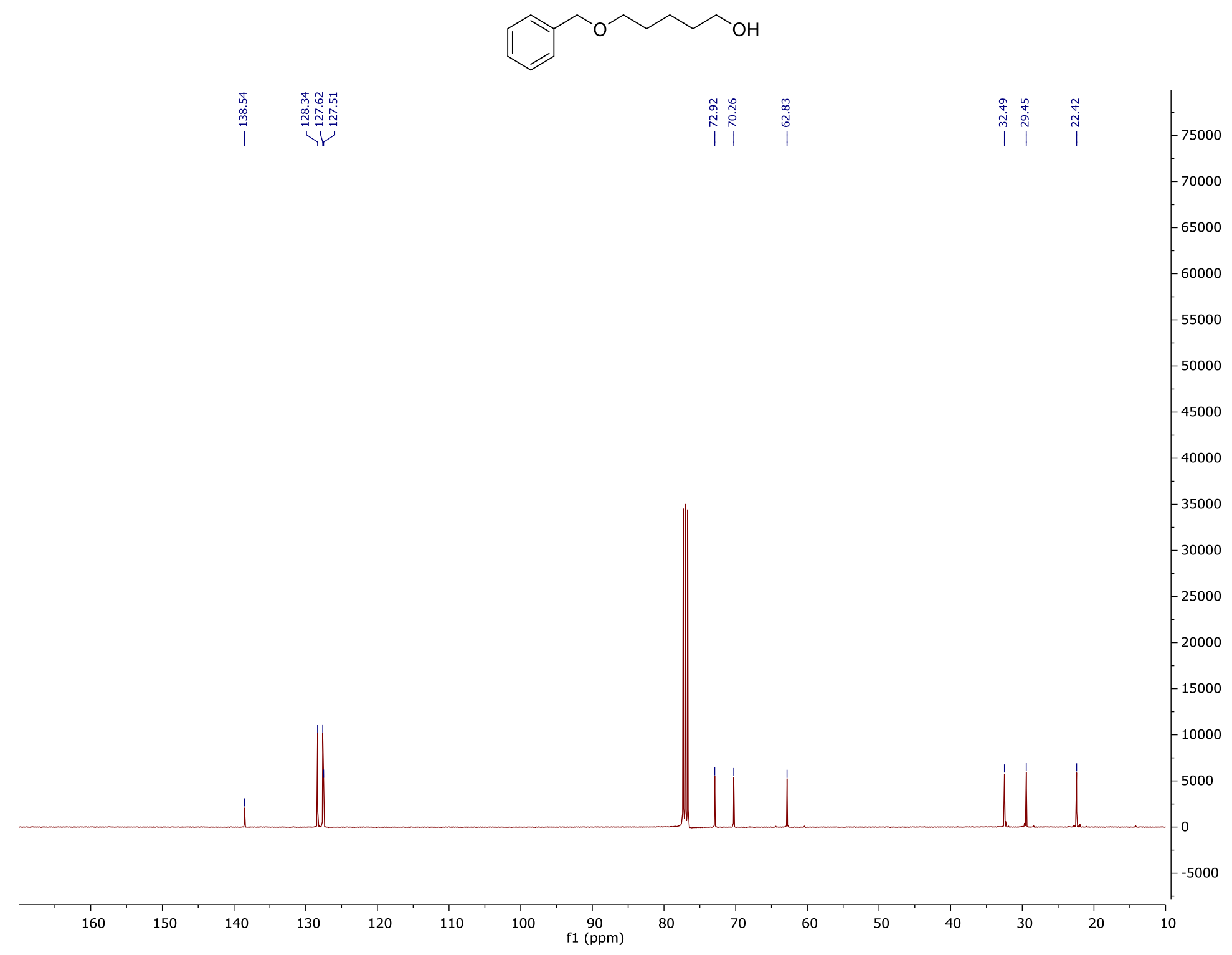




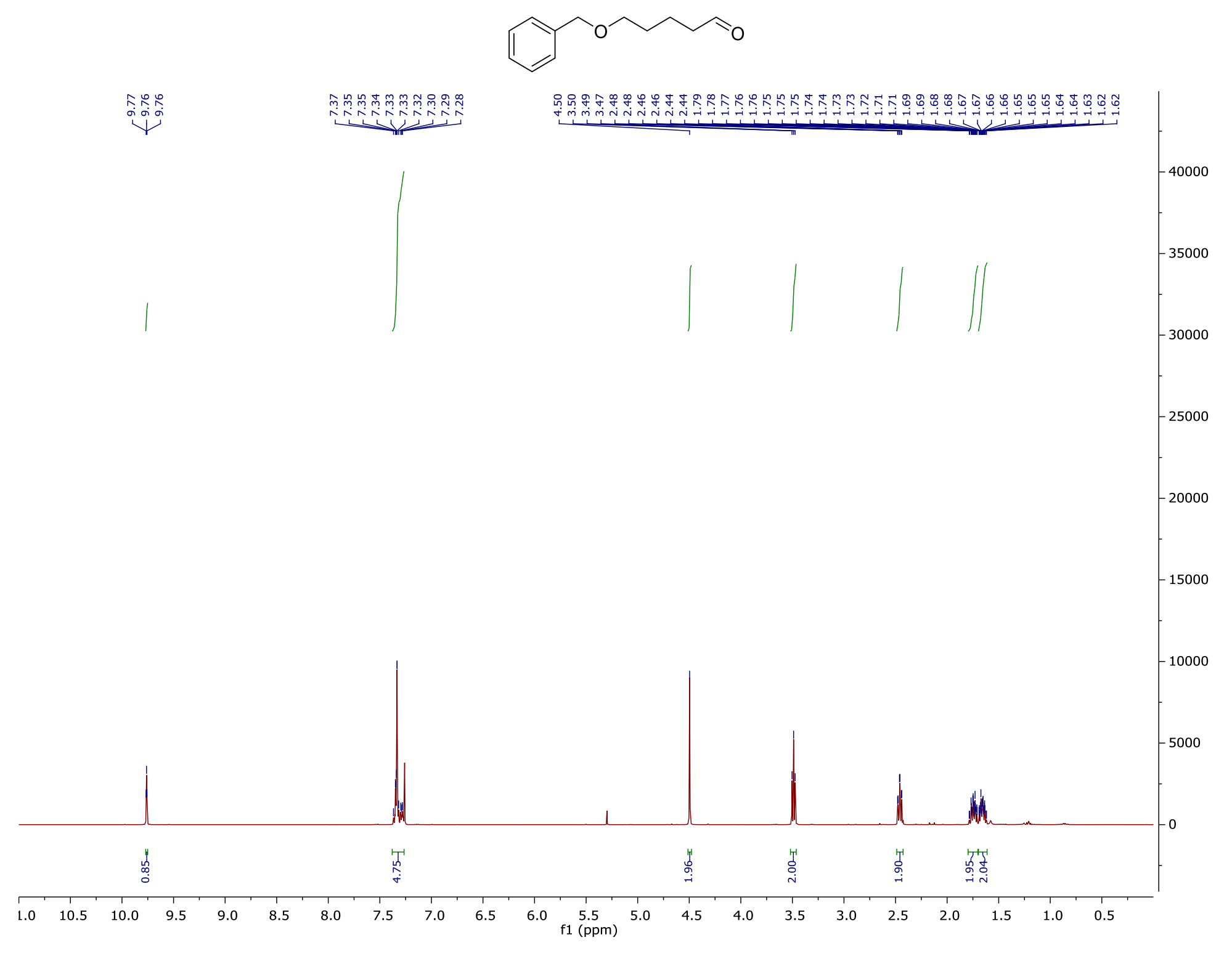




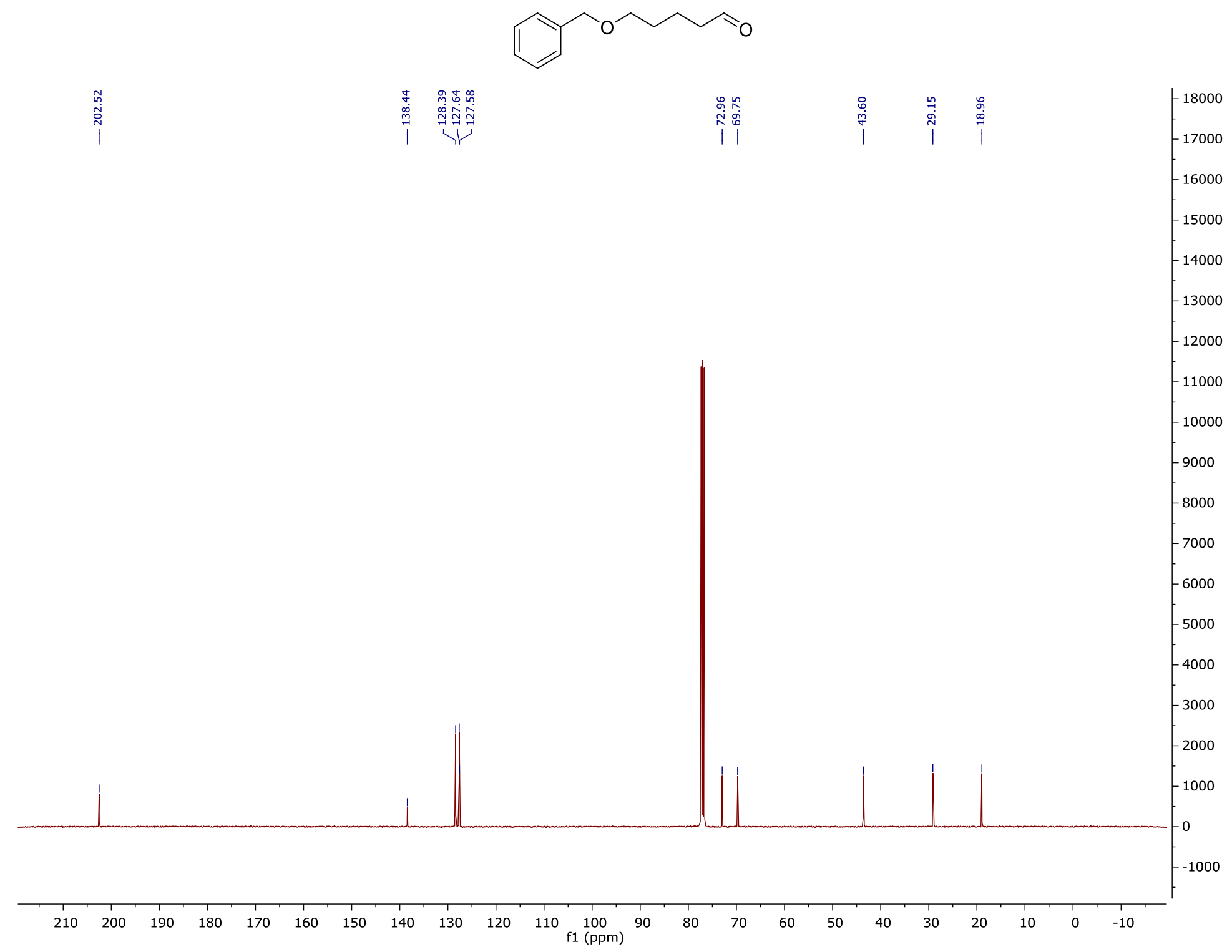




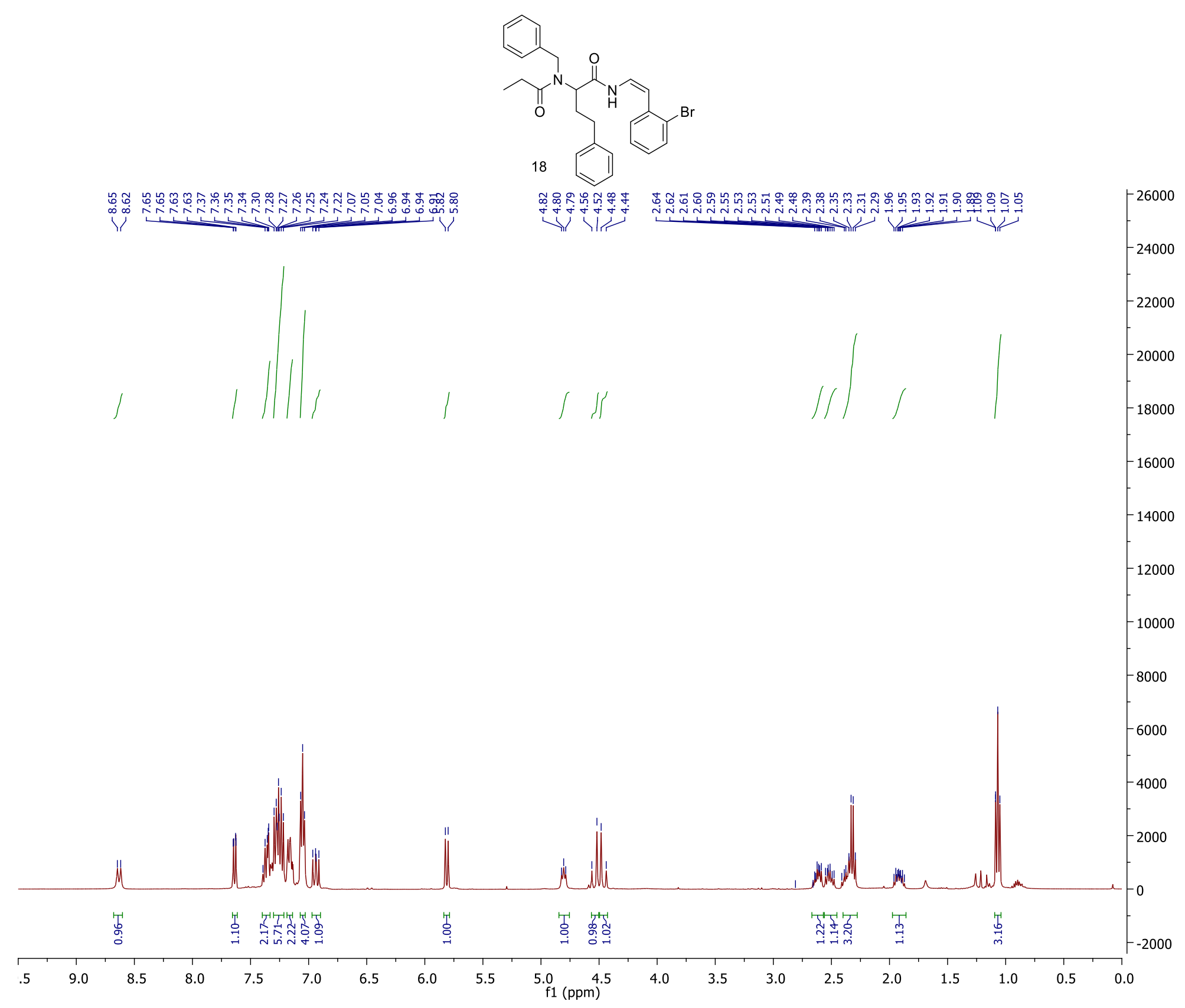




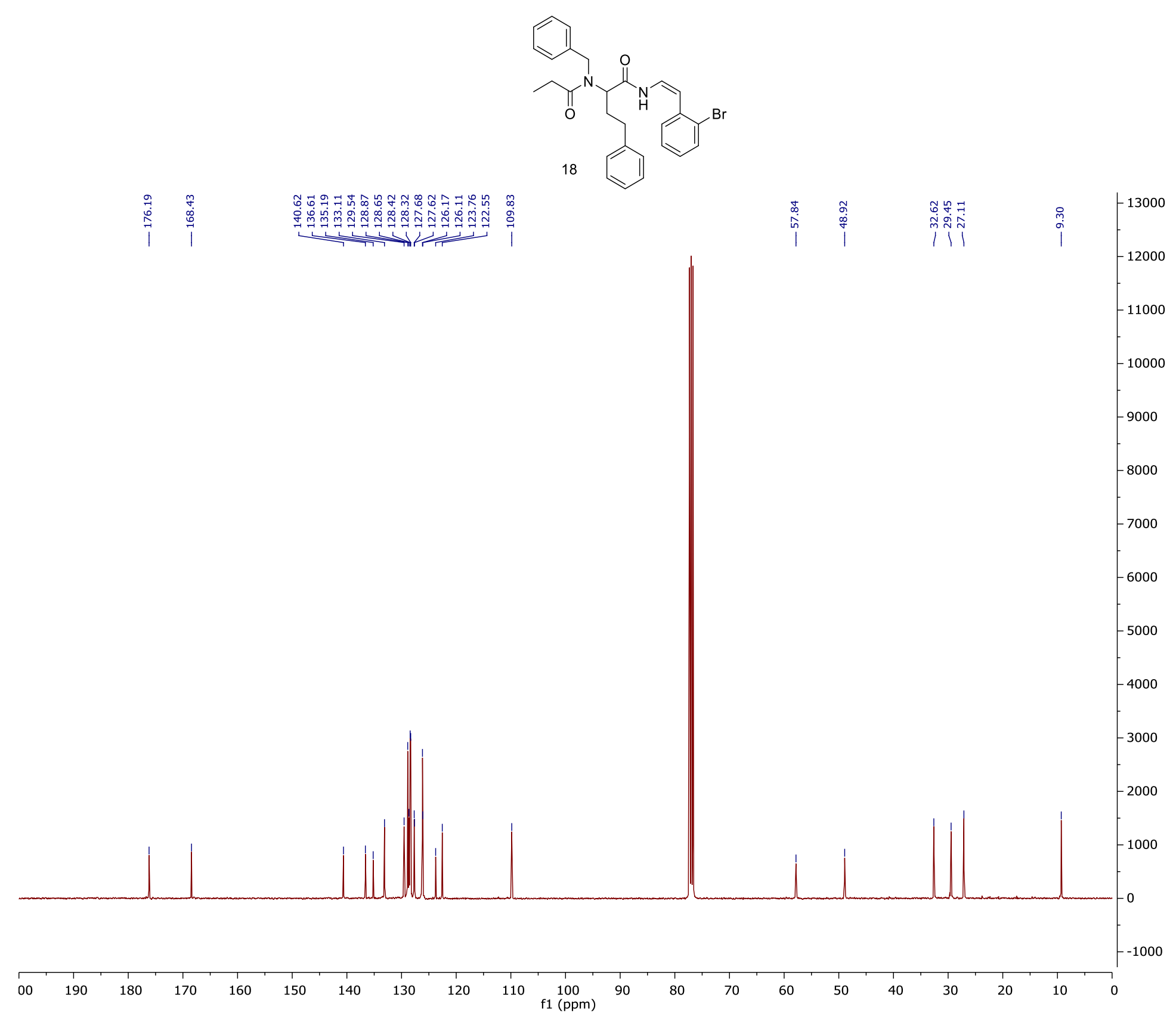




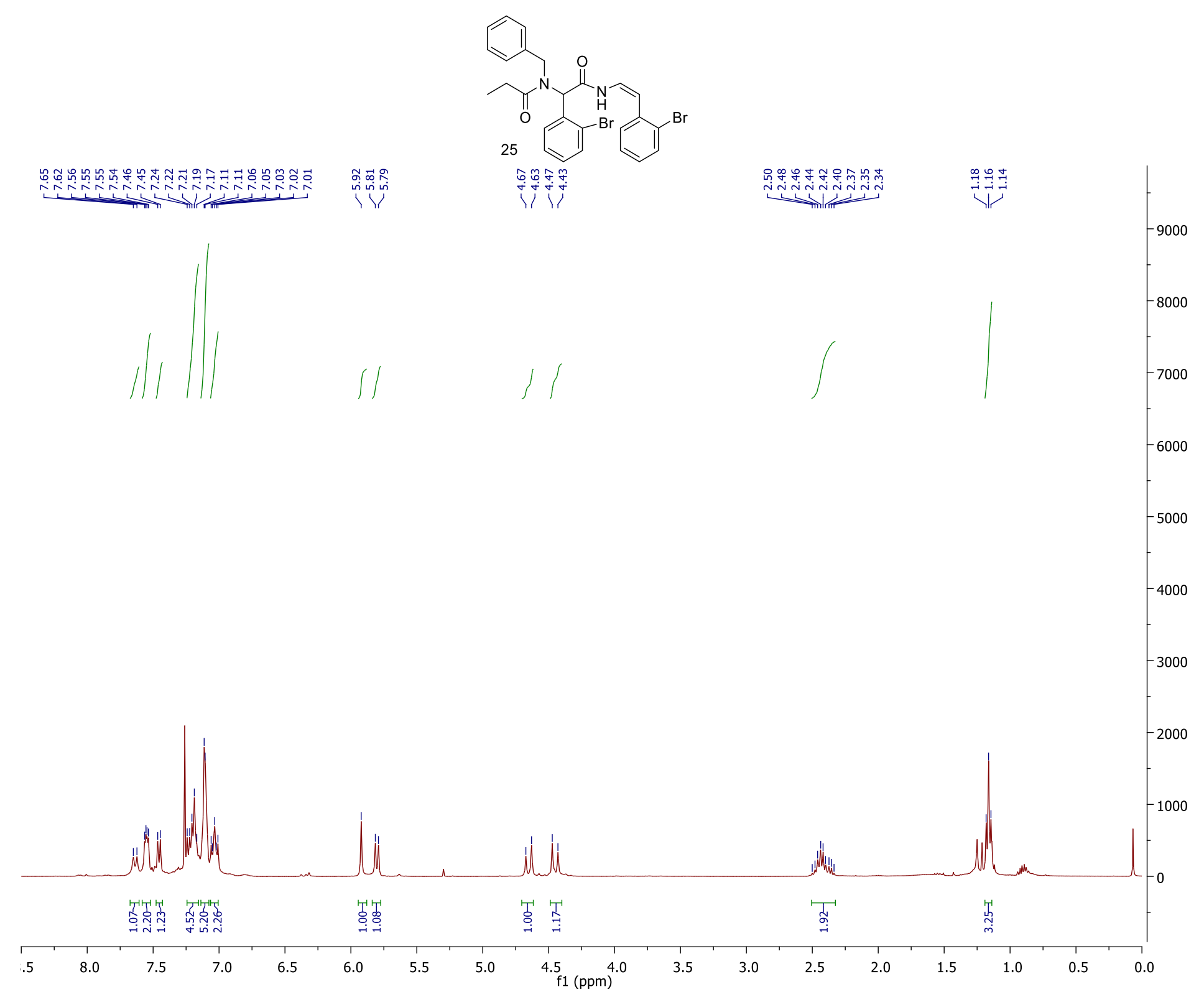




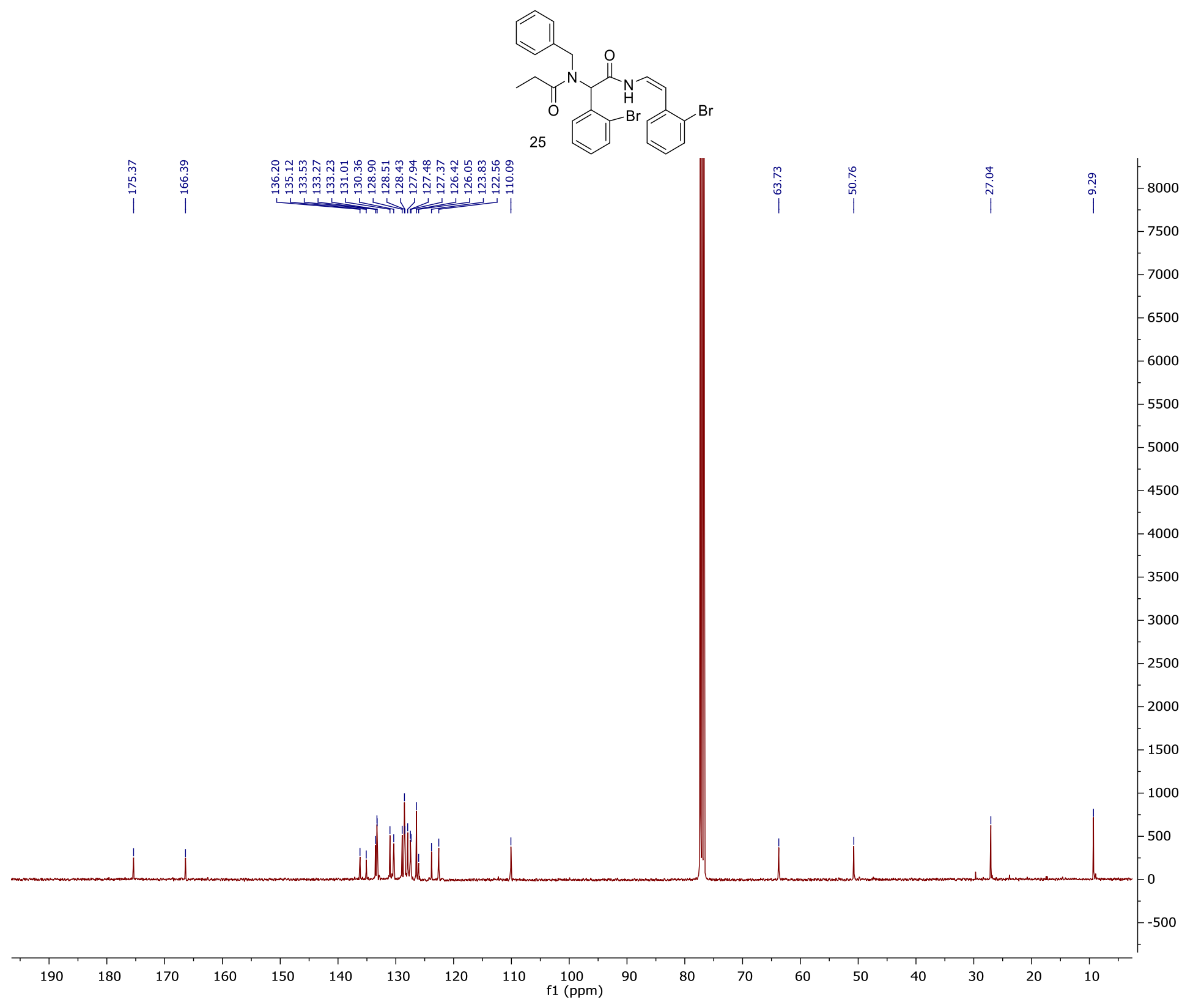

S92 


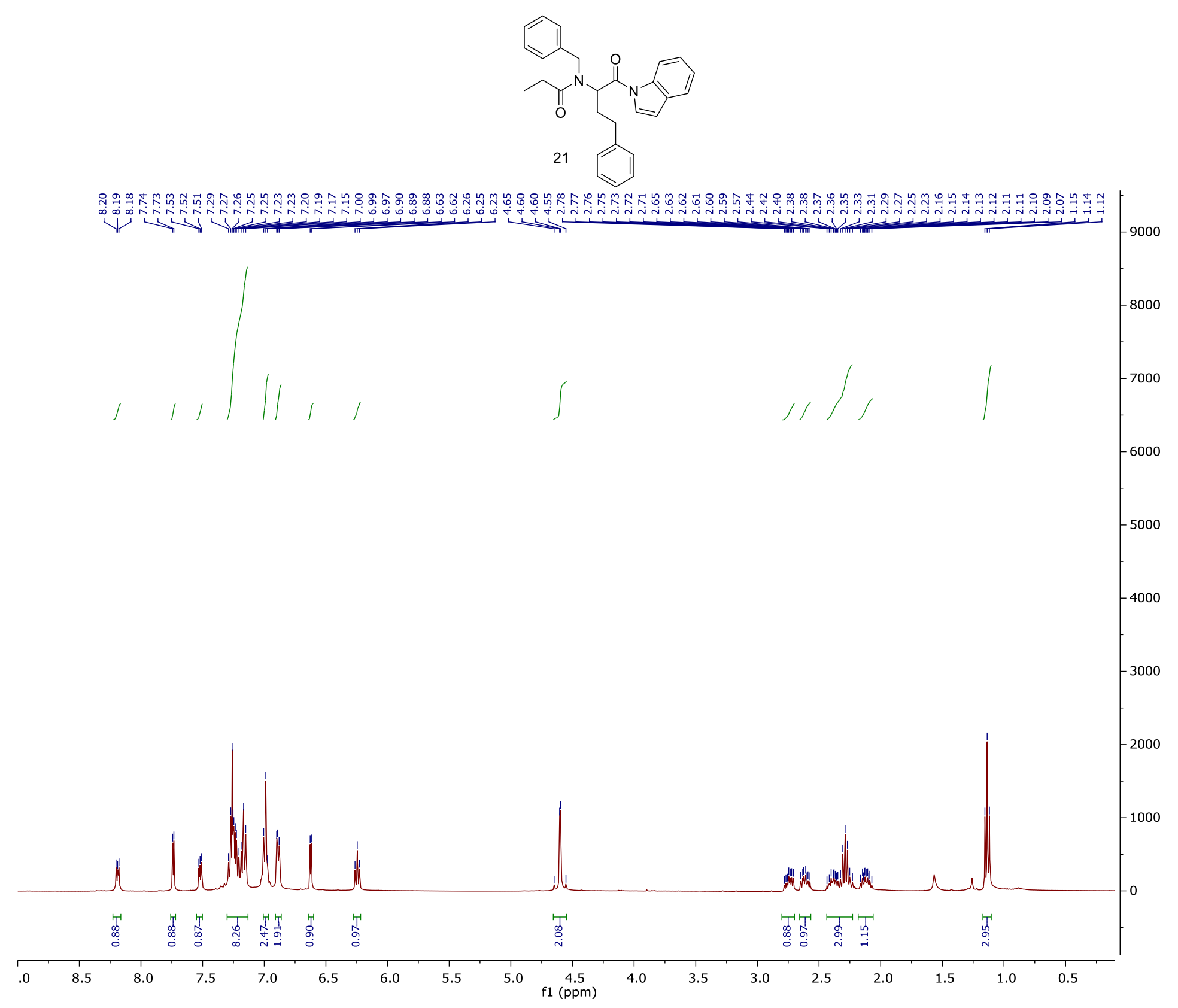




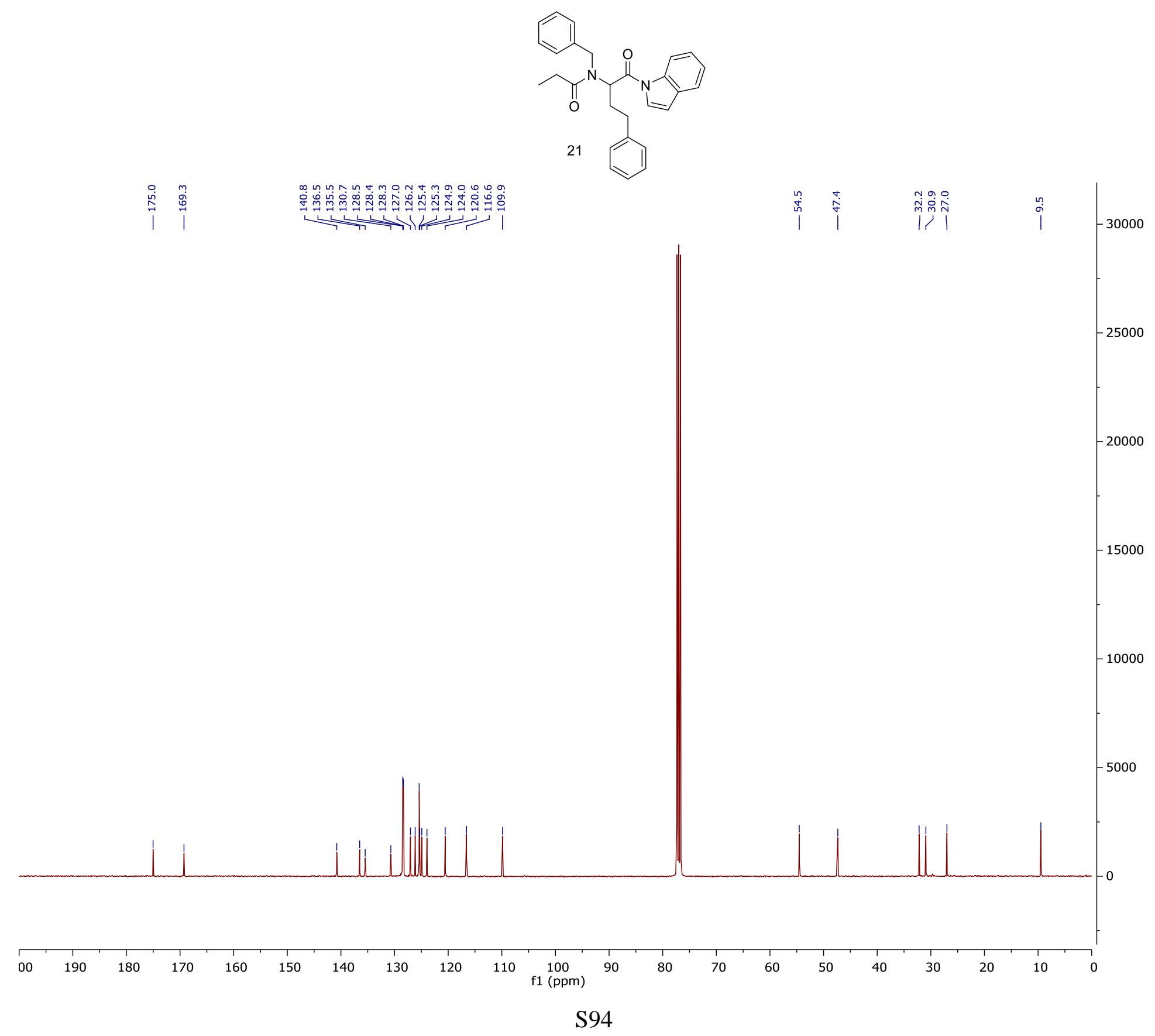




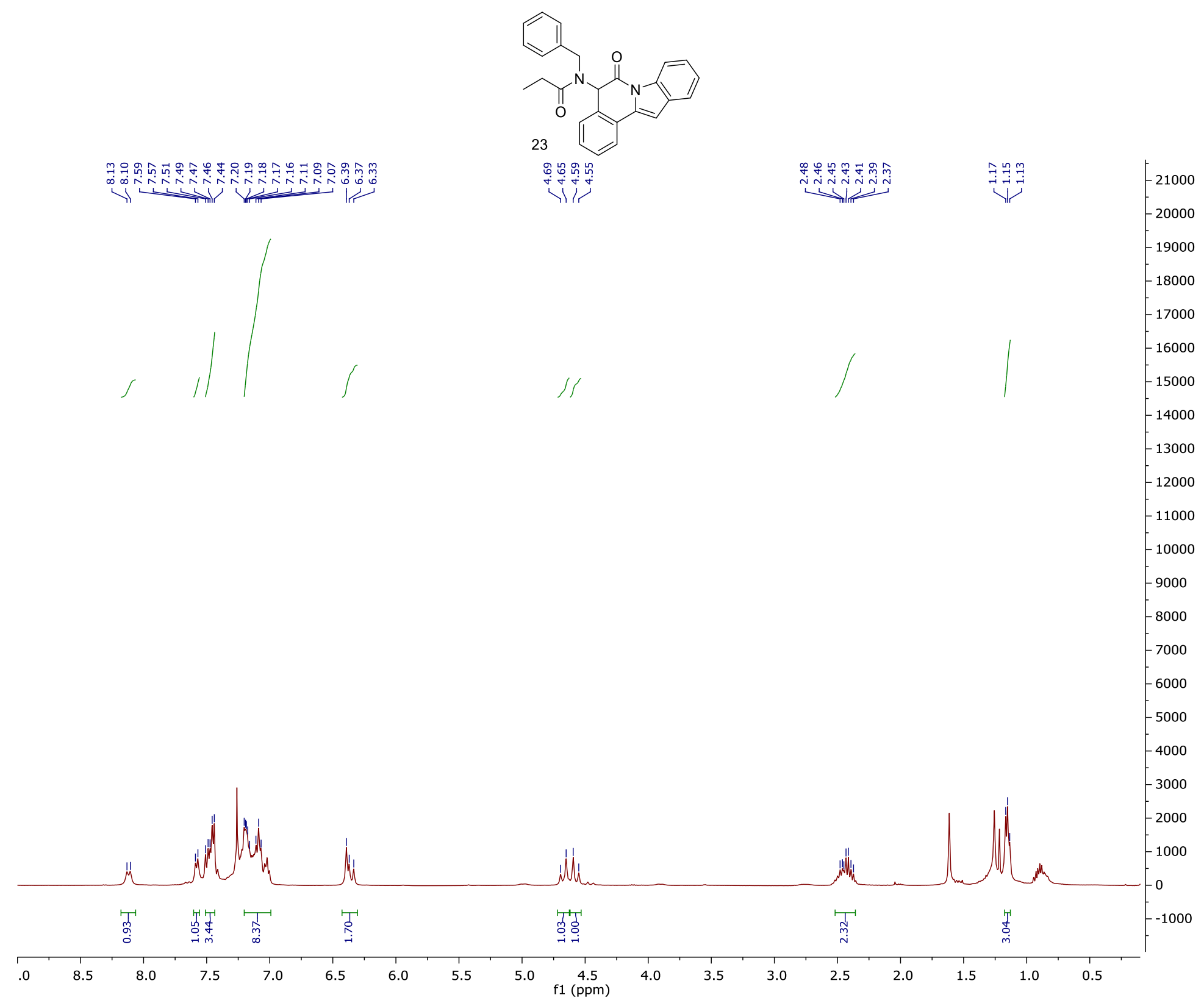




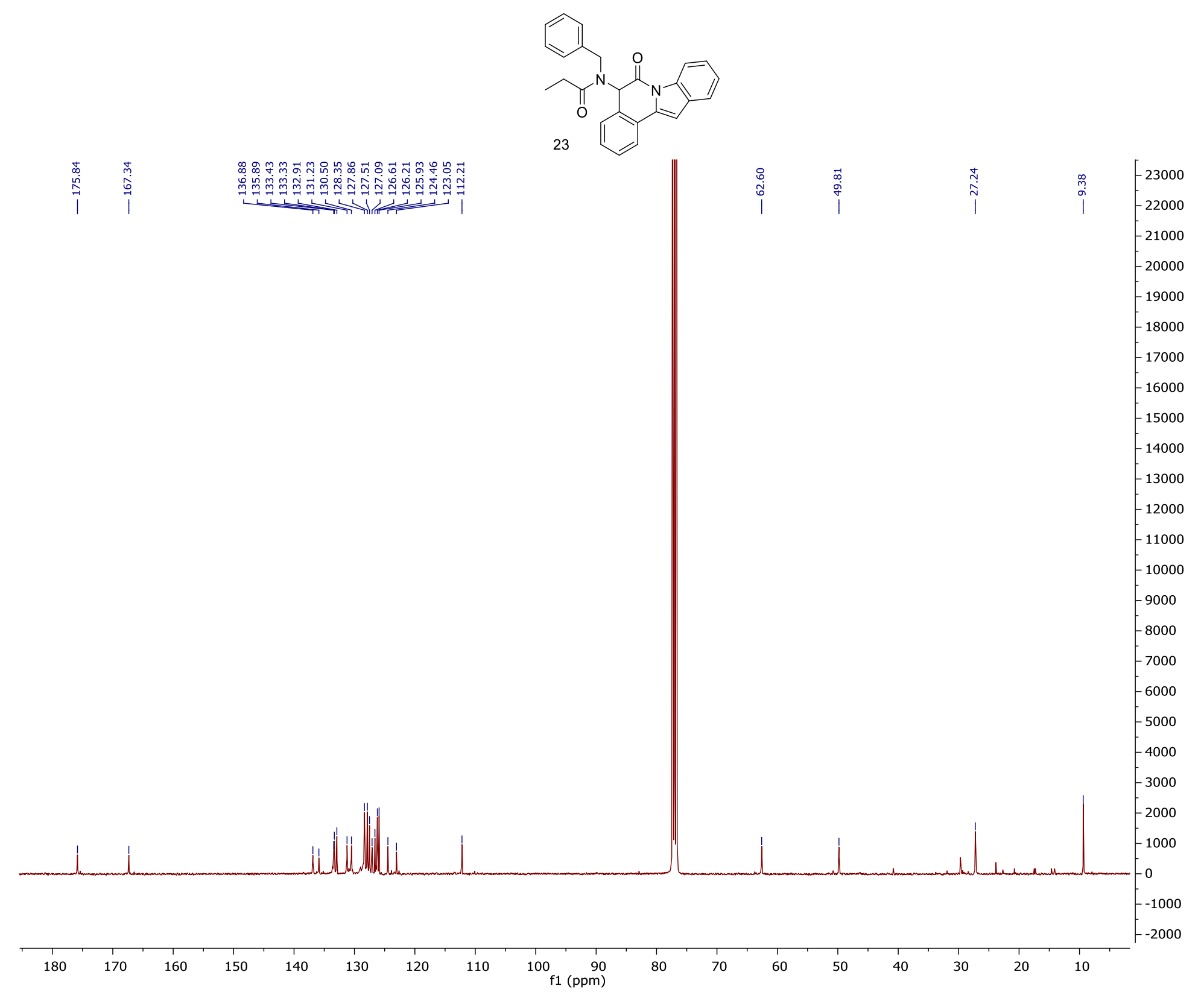




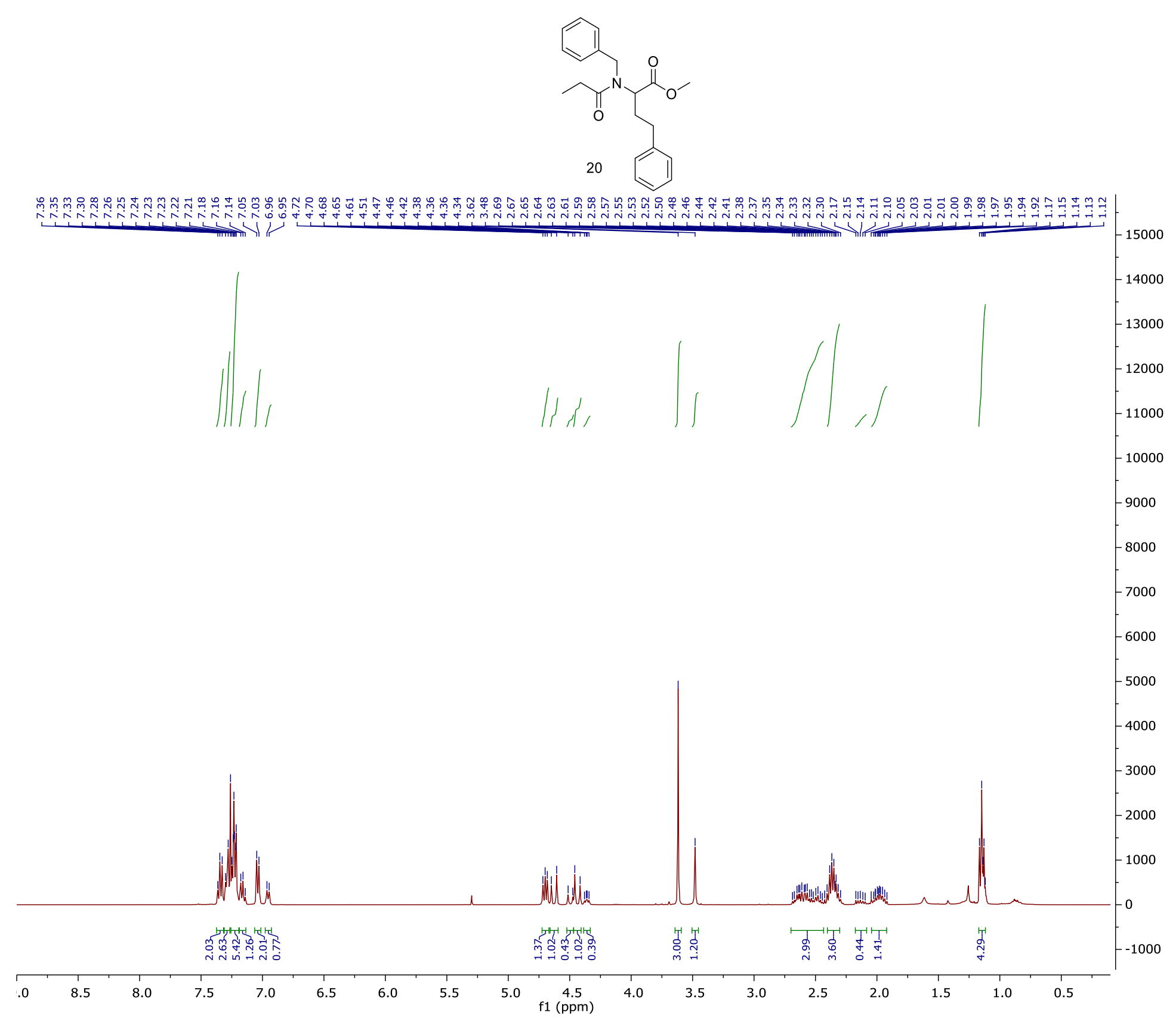




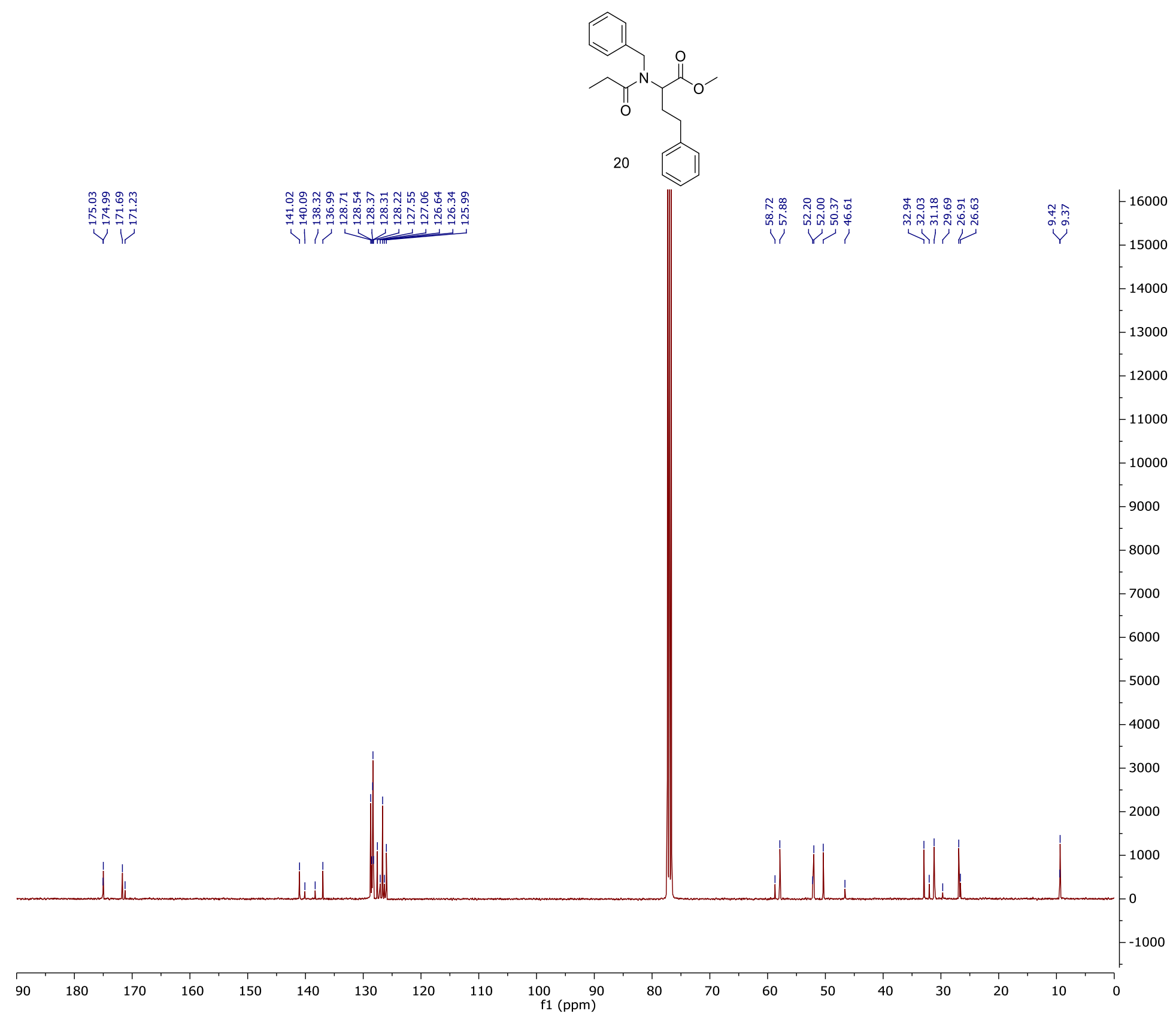




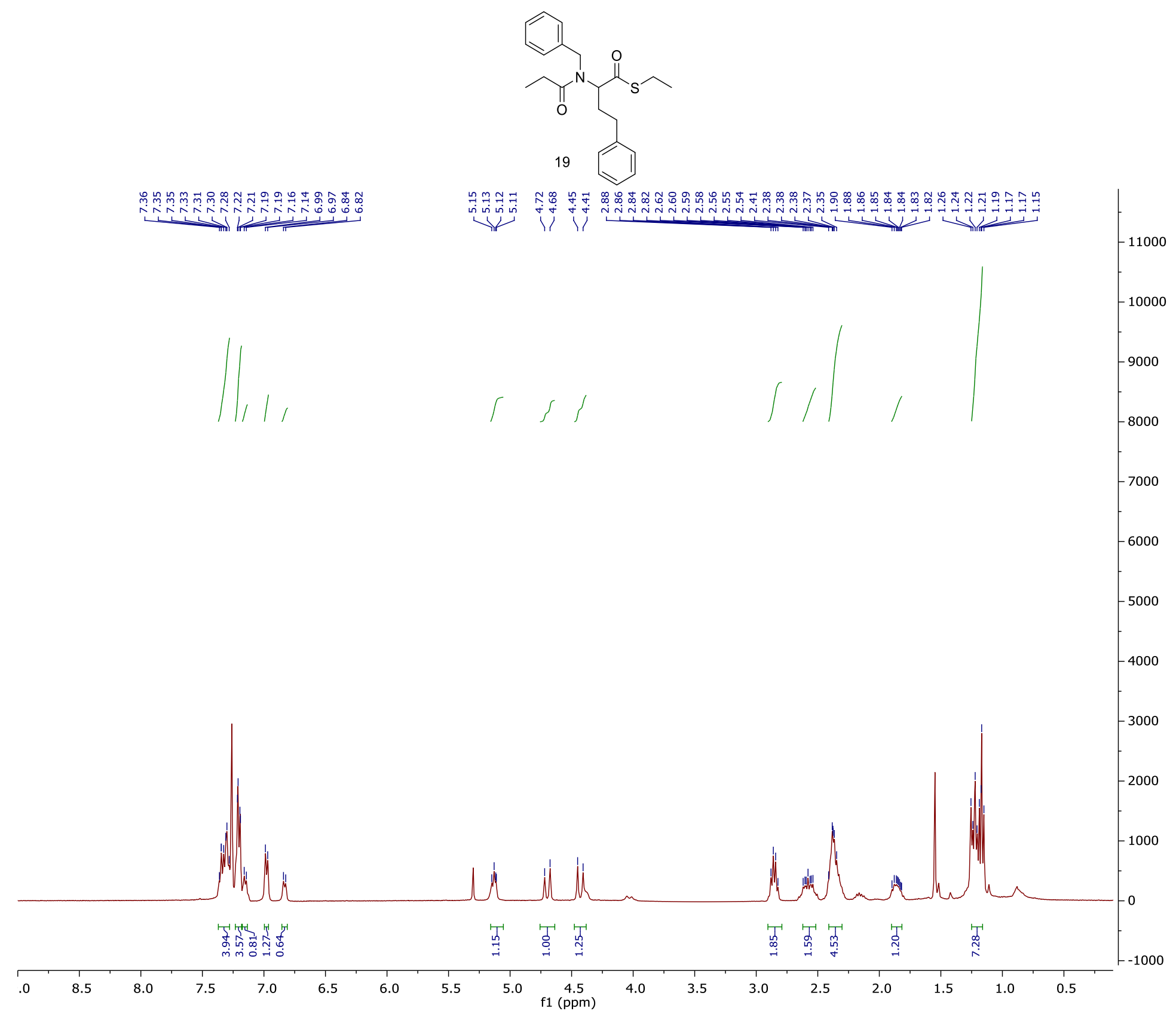




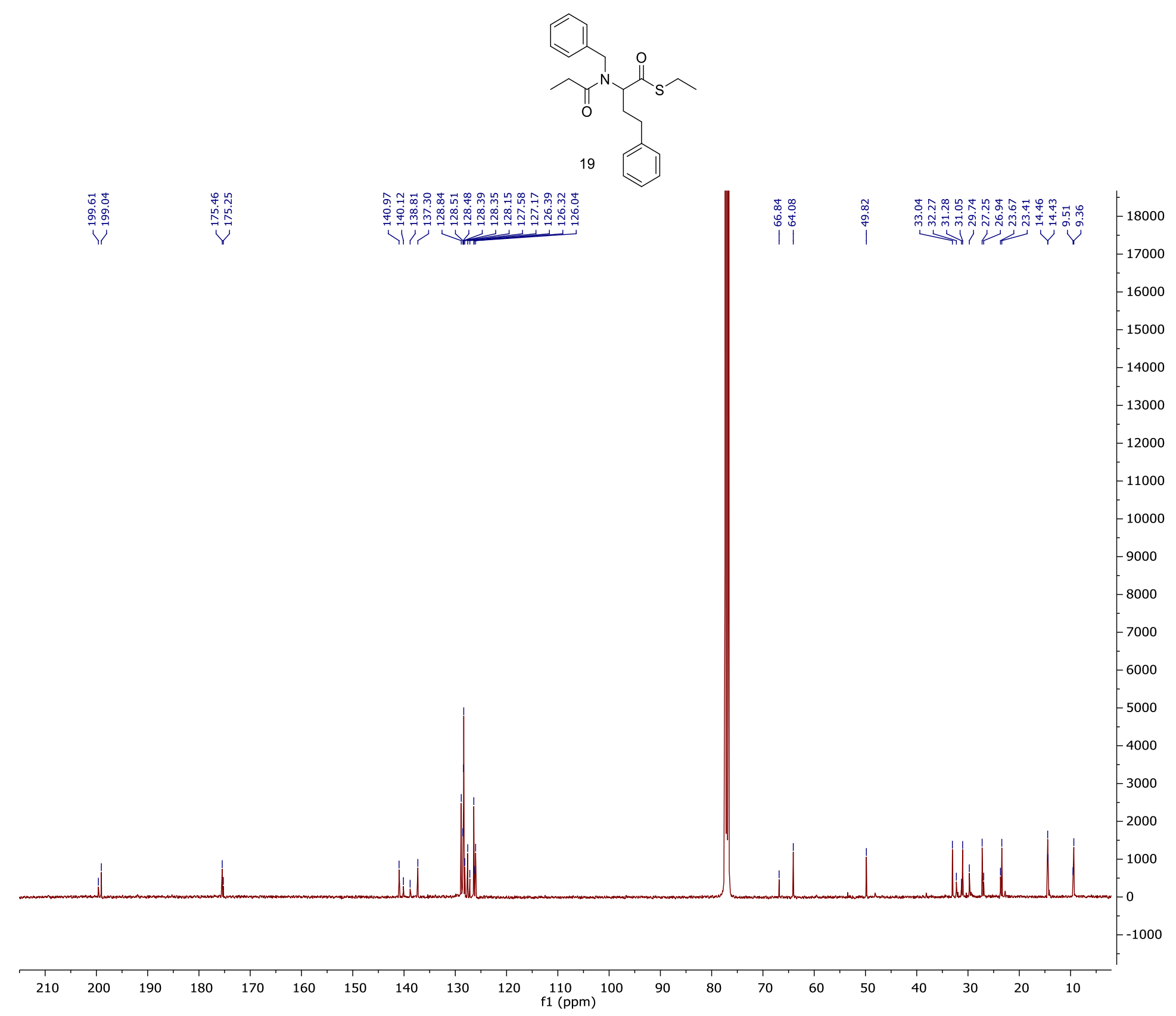




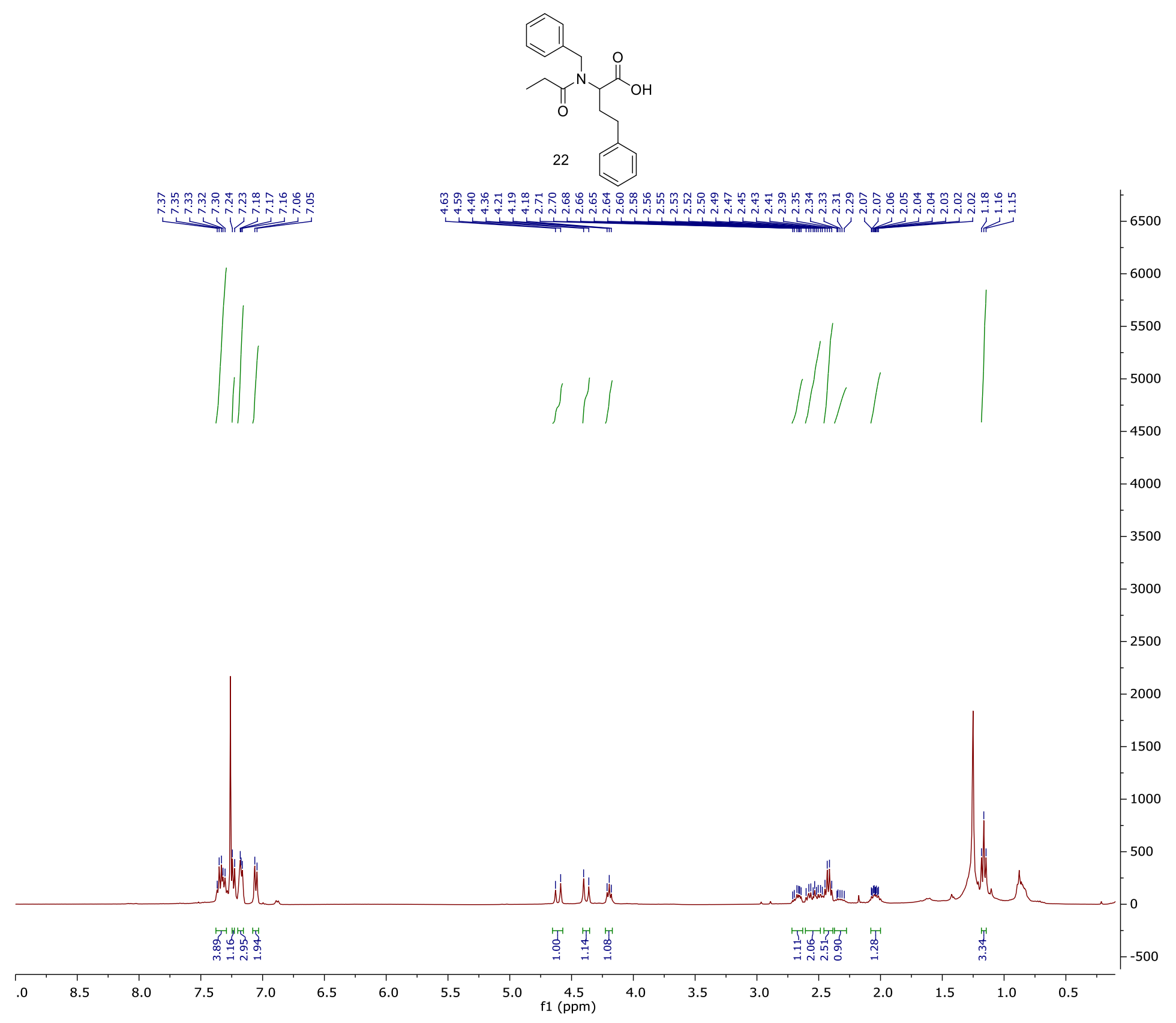




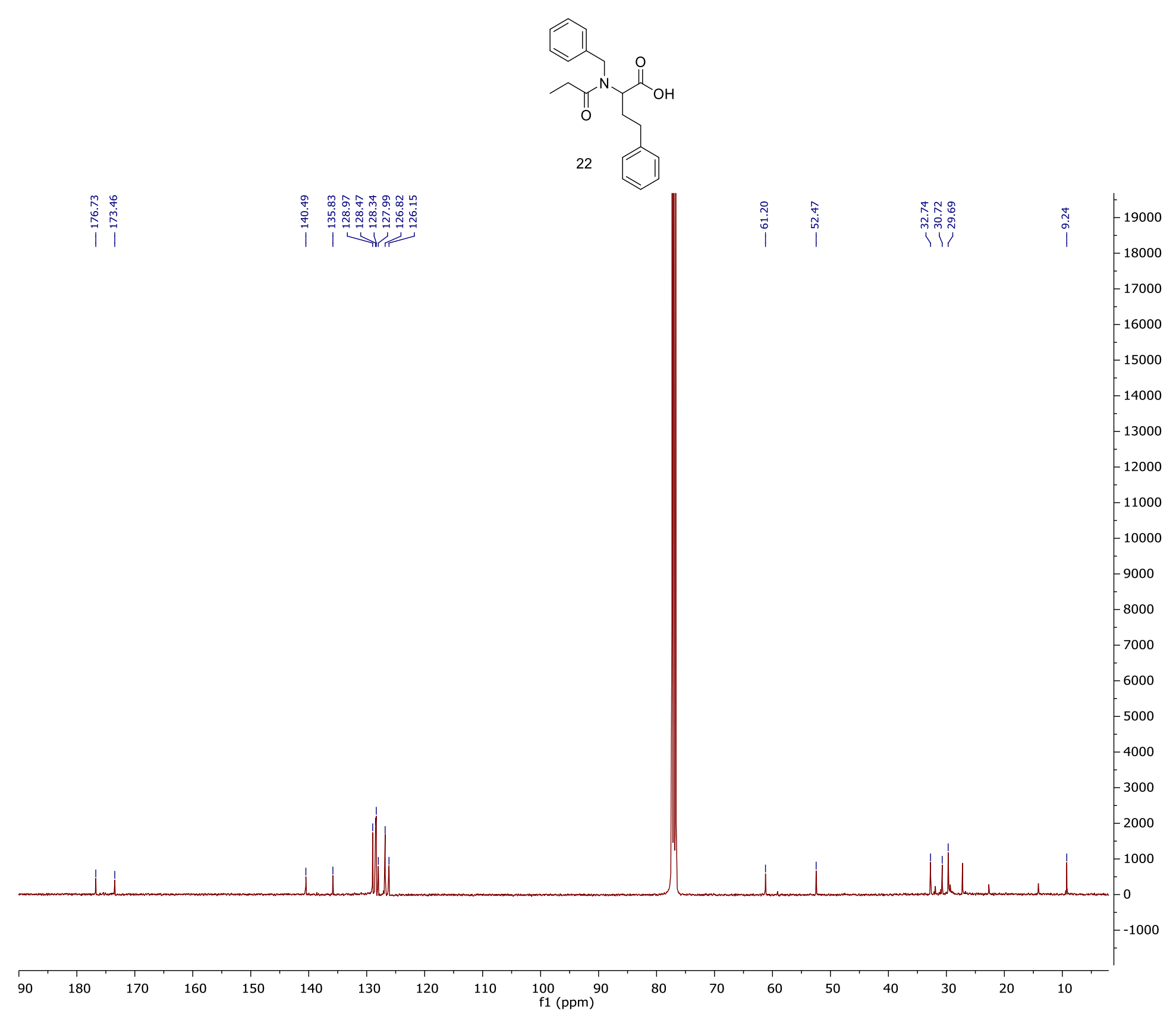

